\title{
Database for Inelastic Collisions of Sodium Atoms with Electrons, Protons, and Multiply Charged Ions
}

\author{
K. Igenbergs ${ }^{\text {a }}$ J. Schweinzer ${ }^{b}$ I. Bray ${ }^{c}$ D. Bridi ${ }^{a}$ F. Aumayr ${ }^{a}, *$ \\ ${ }^{\mathrm{a}}$ Insititut für Allgemeine Physik, Technische Universität Wien, Wiedener Hauptstrasse 8-10, A-1040 Vienna, Austria \\ ${ }^{\mathrm{b}}$ Max-Planck-Institut für Plasmaphysik, Boltzmannstrasse 2, D-85748 Garching, Germany \\ ${ }^{\mathrm{c}}$ Curtin University of Technology, GPO Box U1987 Perth WA 6845, Australia \\ Contents \\ 1 Introduction \\ 2.1 Convergent Close-Coupling for Electron-Impact Collisions 2 \\ 2.2 Atomic-Orbital Close-Coupling for Ion-Impact Collisions 3 \\ 3 Presentation of the Data 3 \\ 3.1 General Remarks on Credibility and Weighted Fitting 3 \\ 3.2 Collisions with Electrons 4 \\ 3.3 Collisions with Protons 7 \\ 3.4 Scaling Relations for Collisions with Multiply Charged Ions 9 \\ 4 Acknowledgements 13 \\ References 13 \\ 5 Explanation of Tables 16 \\ 6 Explanation of Graphs 16 \\ 7 Tables 1 \\ 8 Graphs Graphs - 1 \\ 8.1 Electron-Impact Target Excitation Cross Sections Graphs - 1 \\ 8.2 Electron-Impact Target-Ionization Cross Sections 15 \\ 8.3 Proton-Impact Target-Excitation Cross Sections $\quad$ Graphs - 19 \\ 8.4 Proton-Impact Target-Electron-Loss Cross Sections 33
}

\begin{abstract}
The available experimental and theoretical cross section data for inelastic collision processes of ground (3s) and excited $(3 \mathrm{p}, 4 \mathrm{~s}, 3 \mathrm{~d}, 4 \mathrm{p}, 5 \mathrm{~s}, 4 \mathrm{~d}, 4 \mathrm{f})$ state $\mathrm{Na}$ atoms with electrons, protons, and multiply charged ions have been collected and critically assessed. In addition to existing data, electron-impact cross sections, for both excitation and ionization, have been calculated using the convergent close-coupling approach. In the case of proton-impact the database was enlarged by new atomic-orbital close-coupling calculations. Both electron-impact and proton-impact processes include excitation from the ground state and between excited states $(n=3-5)$. For electron-impact also ionization from all states is considered. In the case of proton-impact electron loss cross sections (the sum of ionization and singleelectron charge transfer) are given. Well-established analytical formulae used to fit cross sections, earlier published by Wutte et al. and Schweinzer et al. for collisions with lithium atoms, were adapted to sodium. The "recommended cross sections" for the considered processes have been critically evaluated and fitted using the adapted analytical formulae. For each inelastic process the determined fit parameters are tabulated. We also present the assessed data in graphical form. The criteria for comprehensively evaluating the accuracy of the experimental data, theoretical calculations, and procedures used in determining the recommended cross sections are discussed.
\end{abstract}




\section{Introduction}

The work on this database is motivated not only by the fundamental interest in the matter, but also by the need for reliable cross sections for the evaluation of plasma edge diagnostics in magnetically confined fusion plasmas by means of active neutral sodium beam spectroscopy. The basic experimental, theoretical, and computational concepts are based on and refer to the earlier work on neutral lithium beam diagnostics [1]- [8]. Similar to lithium, injection of a fast neutral sodium beam into the edge of a magnetically confined plasma is able to deliver diagnostic information such as radial electron density profiles and spatially and time resolved impurity ion concentrations [9]-[11]. A series of recent experiments at the tokamak ASDEX Upgrade and their preliminary analysis have shown that sodium beams are even better suited for this purpose than lithium. The interpretation of the emission line $\mathrm{Na}(3 \mathrm{p}-3 \mathrm{~s})$ of injected atoms, which provides information on the plasma edge density, requires a good knowledge of all involved inelastic collision processes. Therefore, the success of this plasma edge diagnostic method strongly depends on the availability of reliable cross sections of inelastic collisions of sodium atoms with plasma particles namely electron, protons, and multiply charged impurity ions.

The scope of the presented cross section database for collision processes of sodium atoms is mainly determined by the requirements of the Na-beam diagnostics. For the modeling of the diagnostic Na beam eight bound $\mathrm{Na}$ states, 3s, 3p, 4s, 3d, 4p, 5s, 4d, 4f are sufficient. The inelastic collision processes involving these eight $\mathrm{Na}$ states, are electron-impact target excitation, electron-impact target ionization, proton-impact target excitation, and proton-impact target electron loss. In the following chapters data for these processes will be presented. Sorting according to quantum numbers nl will be applied.

For many processes no cross sections could be found in the literature. Therefore we used advanced theoretical methods in this work to derive accurate cross sections for all inelastic processes involving collisions with $\mathrm{Na}$ atoms, see chapter 2 . These theoretical results are critically assessed by comparing them with the most reliable experimental data available. In all cases good or at least satisfactory agreement between experiment and theory is found. Thus it is

\footnotetext{
* Corresponding author.

Email address: aumayr@iap.tuwien.ac.at (F. Aumayr).
}

plausible to have a comparable level of confidence in the theoretical cross sections were experimental validation is not feasible.

The recommended cross sections for the considered processes are a product of either a critical assessment of available experimental and theoretical data or these new calculations alone. They are presented by giving their analytical fit expressions and tabulating the values of all parameters entering these analytic fits. We also present the recommended cross sections in graphical form together with the cross section data used for their generation.

\section{Basic Description of the Applied Theoretical Methods}

\subsection{Convergent Close-Coupling for Electron-Impact Collisions}

In the past twenty years there has been an enormous progress both in theoretical description of electron-atom scattering and the computational adaptation of these theories. The latter is of course strongly supported by the more and more powerful computational resources which allow large closecoupling basis sets. Here we use the convergent close-coupling method (CCC) developed by Bray and Stelbovics [12]. Briefly this method uses a set of square-integrable basis states, obtained by diagonalizing the target Hamiltonian in an orthogonal $L^{2}$ Laguerre basis, to expand the total wave function of the system. For the sodium target we assume that the frozen-core Hartree-Fock Hamiltonian is a sufficiently accurate representation. Hence, we treat sodium as essentially a one-electron target and electron-sodium scattering as a three-body problem.

The CCC method has already been extensively tested for inelastic electron-sodium scattering [13]. It reproduces closely the very detailed measurements of $3 p$ excitation and the total ionization cross section. Thus, we have very good reasons to believe in the accuracy of the CCC method in evaluating integrated cross sections for all discrete transitions at all energies. The key issue is obtaining convergence for the transitions of interest with increasing number of expansion states [14]. Generally, the smaller the cross section, the larger the calculation necessary to obtain an accurate result. In practice, we choose a sufficiently large set of states to obtain accurately the most important transitions of interest. 


\subsection{Atomic-Orbital Close-Coupling for Ion-Impact Collisions}

For ion-impact cross section calculations, we adopted the semiclassical impact-parameter formalism of the close-coupling (CC) method, assuming straight line trajectories for the projectiles [15]- [17]. The time-dependent electronic wave function is expanded in projectile- and target-centered traveling orbitals which need not necessarily be eigenstates of the corresponding atomic Hamiltonian. Thus, in addition to atomic-orbital (AO), so-called pseudostates (PS) are also included in our two-center expansion model. While AO represent the bound spectrum of the separated atoms of relevance for the considered inelastic collision process, PS are chosen to account for the formation of transient molecular orbitals as well as to represent ionization channels. The CC calculation always starts from a linear combination of states which result from diagonalization of the atomic Hamiltonians within the given set of basis states on each center.

The interaction between the core electrons and the "active" electron is described by a model potentials. The experimental energy level diagram of $\mathrm{Na}$ $(n \leq 5 s)$ is accurately reproduced by the eigenvalues of the model potential in. One-center couplings between projectile states induced by the electric field of the $\mathrm{Na}^{+}$core are calculated in good approximation by assuming a pure Coulomb interaction potential.

For collisions of $\mathrm{H}^{+}$with $\mathrm{Na}(\mathrm{nl})$ cross sections for single electron capture (SEC), target excitation (EXC), and ionization (ION) are mainly derived from $\mathrm{AO}$ calculations involving 44 states centered on the proton core and 83 states (AO44_83) centered on the $\mathrm{Na}^{+}$core. This main basis set is used for calculations with initial $\mathrm{Na}$ target states $3 \mathrm{~s}, 3 \mathrm{p}, 3 \mathrm{~d}$, 4s, and $4 \mathrm{p}$ for impact energies beyween 1 and $500 \mathrm{keV}$. On both centers atomic states with principal quantum numbers $n \leq 5$ are represented in the calculations. All other states are PS, which are orthogonal to the included atomic states of the basis and thus will overlap with higher excited bound states as well as with continuum states. The projection of such discrete non-bound pseudo-states on the continuum is the basis for the calculation of ionization cross sections. Another basis set AO70_29 is used to check the convergence of results as well as to extend the impact energy range towards $0.2 \mathrm{keV}$. In addition to the two-center calculations, pure one-center calculations without projectile centered states (AO0_83) were also performed. These calculations were used to derive EXC and ION cross sections at impact energies of $200-1000 \mathrm{keV}$ with considerable less computational effort. In this high-impact-energy region the EXC and ION process is completely decoupled from electron capture, and thus results from AO0_83 do not differ from those of the much more elaborate calculation AO44_83.

Cross sections for target electron loss (ELOSS, that is $\mathrm{SEC}+\mathrm{ION}$ ) are obtained by summing over all cross sections for population of projectilecentered states and the direct ionization cross section of the $\mathrm{Na}$ atom. Results for ELOSS from the AO44_83 calculations are believed to be convergent for impact energies of 1-15 keV. For higher impact energies the ELOSS cross section is dominated by the ionization process, which is not sufficiently well described in the basis set applied here. Therefore, at high impact energies ELOSS cross sections are underestimated by our AO calculations. This was taken into account in the process of establishing recommended cross sections (see section 3.3).

In general, the coupled equations are solved along straight line trajectories $R=v t+b(R$ being the internuclear distance and $v$ the velocity) from $v t=-300 a_{0}$ to $v t=300 a_{0}\left(a_{0}\right.$ being the Bohr radius) and for impact parameters $b$ from 0.3 to 50 $a_{0}$. However, for dipole-allowed EXC cross sections with small $\Delta E$ values much larges meshes had to be used.In particular, the AO0_83 calculations were done between $v t=-800 a_{0}$ and $v t=800 a_{0}$ with impact parameters up to $200 a_{0}$.

Similar calculations were performed for $\mathrm{He}^{2+}$ and $\mathrm{Be}^{4+}$ colliding with $\mathrm{Na}(3 \mathrm{~s}, 3 \mathrm{p})$. Results from these calculation were used to check the behavior of EXC and ELOSS cross section with respect to the projectile charge q (see section 3.4).

\section{Presentation of the Data}

\subsection{General Remarks on Credibility and Weighted Fitting}

The most important excitation and ionization cross sections induced by the collisions of both electron and proton impact on sodium have been subject to a large number of experimental and theoretical studies. Obviously, the various methods imply different margins of error. For a comprehensive evaluation of the cross sections it became necessary to judge the credibility and to weight 
the cross sections accordingly in the fit leading to recommended cross section. The following general guidelines were applied to this process. First, it is a known fact that First-Order Plain Wave Born Approximation (PWBA) cross sections excellently predict the asymptotic cross sections in the high energy tail and but show a large discrepancy in the low energy region. Thus, PWBA cross sections were weighted strongly above a certain energy limit and very poorly below this limit. Second, the better the data were compatible with the general trend of all collected cross sections of one transition the stronger they were weighted in the fit. Third, data predating 1980 were generally considered to be not as accurate as more recent ones.

\subsection{Collisions with Electrons}

The presented database consists of comprehensively evaluated data found in the scientific literature. The quality and reliability of the database is further enhanced by including the CCC calculations into the critical data assessment process. All calculations are performed in the impact-energy range from the excitation threshold up to $10 \mathrm{keV}$.

Experimental data exist for electron-impact excitation (EXC) processes from the ground state to $3 \mathrm{p}, 3 \mathrm{~d}, 4 \mathrm{~s}, 4 \mathrm{p}, 4 \mathrm{~d}$, and $5 \mathrm{~s}$. Additionally experimental data for $e^{-}+N a(3 p) \rightarrow e^{-}+N a(3 d)$ is available.

Representation of the cross sections is achieved by applying the Levenberg-Marquardt method for non-linear fits [54] implemented in gnuplot [55] and using the following analytic expression with seven parameters $\left(A_{1}, \ldots, A_{7}\right)$ :

$$
\begin{gathered}
\sigma_{e^{-}}^{E X C}(e / e V)\left[\mathrm{cm}^{2}\right]=\frac{A_{7} \cdot 10^{-16}}{E}\left[\frac{E-\Delta E}{E}\right]^{A_{6}} . \\
\cdot\left[\sum_{j=1}^{4} \frac{A_{j}}{(E / \Delta E)^{j-1}}+A_{5} \cdot \ln \left(\frac{E}{\Delta E}\right)\right] \\
\forall E>\Delta E
\end{gathered}
$$

where $\Delta E$ is the excitation threshold energy. The fitting functions were chosen to assure asymptotically correct behavior both at low and high impact energies. The behavior in the low energy region is mainly implemented by the second factor. It is thus essentially necessary that parameter $A_{6}$ does not take negative values. As a typical example for the quality of the fitting procedure the result of the $\mathrm{CCC}$ calculation for a single EXC process $(\mathrm{Na}(3 s \rightarrow 3 d))$ is presented in Fig.1 together with the fit parameters according to Tab.1. Deviations of the fit from the defining data points are in general below a few percent. In some cases there are data available for energies just above, but very close to the excitation threshold energy. The fits, though, all converge toward zero at threshold.

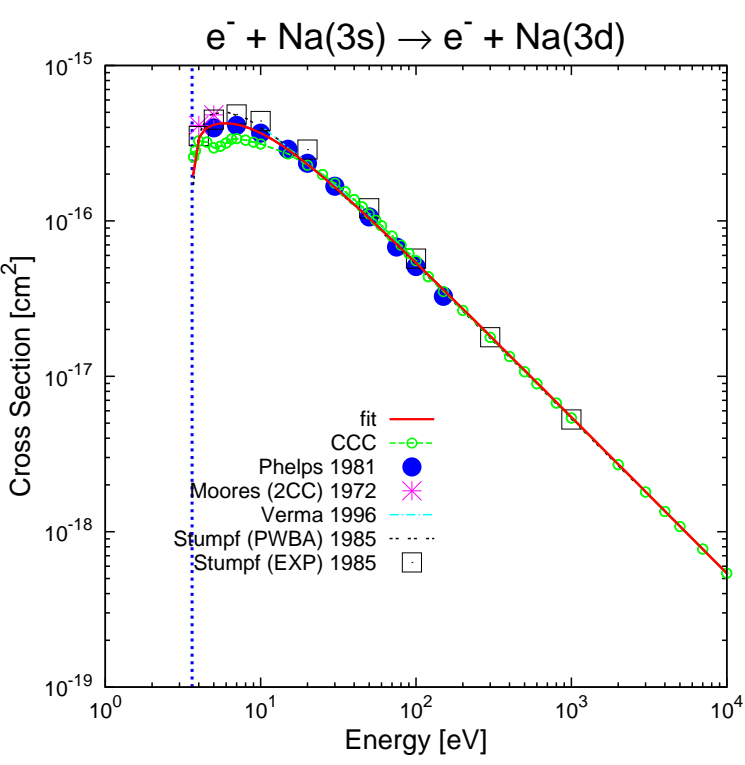

Fig. 1. Results from the CCC calculations for the $\mathrm{Na}(3 s \rightarrow 3 d)$ electron-impact excitation in comparison with experimental and other theoretical data and the analytic fit (see Eq.(1) and Tab.1)

The collections of cross sections including the present CCC calculations are visualized in graphs in section 8.1. In these graphs the excitation threshold energy of the corresponding transition is displayed by a vertical blue single-dashed line. The accuracy of the experimental and theoretical data is limited according to the terms in chapter 3.1.

The collection of electron-impact target ionization (ION) cross sections includes additional data only for ground state and first excited state ionization. Again, the deviations of the fit and the data points are essentially below a few percent, except very close to the threshold. This gave rise to the introduction of the low energy limit $\mathrm{E}_{\text {low }}$, below which the fit cannot predict the trend of the cross section anymore. Thus, the final form of the used analytical fit formula for electron-impact target ionization with seven fit parameters $\left(A_{1}, \ldots, A_{7}\right)$ is 


\begin{tabular}{|c|c|c|c|c|c|}
\hline \multicolumn{6}{|c|}{ Electron-impact target excitation } \\
\hline Transition & Name & Published in & Referenced in & Weight in fit & Comment \\
\hline \multirow[t]{12}{*}{$3 \mathrm{~s} \rightarrow 3 \mathrm{p}$} & Buckman 1979 & {$[18]$} & [19] & Low & Experiment \\
\hline & Enemark 1972 & {$[20]$} & - & High & Experiment \\
\hline & Gould 1970 & {$[21]$} & {$[22]$} & Low & Calculation \\
\hline & Karule 1970 & {$[23]$} & {$[22]$} & Low & Experiment \\
\hline & Kim 2001 & {$[24]$} & - & Moderate & Below $100 \mathrm{eV}$ : very low \\
\hline & & & & & Calculation \\
\hline & Mitroy 1987 & {$[19]$} & - & Low & Calculation \\
\hline & Moores (2CC) 1972 & {$[22]$} & - & Low & Two center close-coupling calculation \\
\hline & Moores (4CC) 1972 & {$[22]$} & - & Low & Four center close-coupling calculation \\
\hline & Phelps 1981 & {$[25]$} & - & High & Experiment \\
\hline & Srivastava 1980 & {$[26]$} & - & Moderate & Experiment \\
\hline & Zapesochnyi 1976 & {$[27]$} & {$[28]$} & Very low & Experiment \\
\hline \multirow{5}{*}{$3 \mathrm{~s} \rightarrow 3 \mathrm{~d}$} & Moores (2CC) 1972 & {$[22]$} & - & Low & Two center close-coupling calculation \\
\hline & Phelps 1981 & {$[25]$} & - & High & Experiment \\
\hline & Stumpf (PWBA) 1985 & {$[29]$} & - & Moderate & Born approximation calculation \\
\hline & Stumpf (EXP) 1985 & {$[29]$} & - & High & Experiment \\
\hline & Verma 1996 & {$[30]$} & - & Low & Calculation \\
\hline \multirow[t]{3}{*}{$3 \mathrm{~s} \rightarrow 4 \mathrm{~s}$} & Moores (2CC) 1972 & {$[22]$} & - & Low & Two center close-coupling calculation \\
\hline & Phelps 1981 & {$[25]$} & - & High & Experiment \\
\hline & Srivastava 1980 & {$[26]$} & - & Moderate & Experiment \\
\hline $3 \mathrm{~s} \rightarrow 4 \mathrm{p}$ & Phelps 1981 & {$[25]$} & - & High & Experiment \\
\hline $3 \mathrm{~s} \rightarrow 4 \mathrm{~d}$ & Phelps 1981 & {$[25]$} & - & High & Experiment \\
\hline $3 \mathrm{p} \rightarrow 3 \mathrm{~d}$ & Stumpf 1985 & {$[29]$} & - & Moderate & Experiment \\
\hline
\end{tabular}

Used publications for electron-impact target excitation cross sections. The columns contain the following information: (1) transition in question. (2) name of the data in the graphs. (3) original reference. (4) if applicable the publication where the used data were taken from. (5) weight in fit according to assigned credibility. (6) comment.

\section{Table B}

\begin{tabular}{|c|c|c|c|c|c|}
\hline \multicolumn{7}{|c|}{ Electron-impact target ionization } \\
\hline Ground state & Name & Published in & Referenced in & Weight in fit & Comment \\
\hline $3 \mathrm{~s}$ & Bates 1965 & {$[31]$} & {$[32]$} & Low & Calculation \\
& Fujii, Srivastava 1995 & {$[33]$} & - & Very high & Experiment \\
& Johnston 1995 & {$[34]$} & - & Moderate & Experiment \\
& McGuire 1971 & {$[35]$} & - & Low & Calculation \\
& McGuire 1997 & {$[36]$} & - & Moderate & Calculation \\
& Rakstikas 2001 & {$[37]$} & - & High & Calculation \\
& Tan 1996 & {$[38]$} & - & High & Experiment \\
& Zapesochnyi 1968 & {$[39]$} & {$[38]$} & Low & Experiment \\
\hline 3 3p & Tan 1996 & {$[38]$} & - & High & Experiment \\
\hline
\end{tabular}

Used references for electron-impact target ionization cross sections. For description of column contents see Table A. 


$$
\begin{gathered}
\sigma_{0, e^{-}}^{I O N}(E / e V)\left[\mathrm{cm}^{2}\right]=\frac{A_{7} \cdot 10^{-13}}{E \cdot I_{n l}} . \\
\cdot\left[A_{5} \cdot \ln \left(\frac{E}{I_{n l}}\right)+A_{6} \cdot \exp \left(-\frac{E}{I_{n l}}\right)+\right. \\
\left.+\sum_{j=1}^{4} A_{j} \cdot\left(1-\frac{I_{n l}}{E}\right)^{j-1}\right] \\
\forall E \geq E_{\text {low }}
\end{gathered}
$$

Neither this fit formula nor the CCC calculations take into account that at impact energies above the binding energy of the $2 \mathrm{~s}$ and $2 \mathrm{p}$ subshells inner-shell ionization possibly occurs. We proceeded in the following way: first the available cross section data were collected and fitted and then the contribution of inner-shell ionization was added to the fitting curve. We used the Lotz-Formula (Eq.(3)) that provides an analytical evaluation of inner-shell ionization [56]. In this case the Lotz-formula is outlined explicitely as follows:

$$
\begin{gathered}
\sigma_{2 p}^{I O N}(E / \mathrm{eV})\left[\mathrm{cm}^{2}\right]=q_{2 p} \cdot L_{1} \cdot \frac{\ln \left(E / I_{2 p}\right)}{E \cdot I_{2 p}} . \\
\cdot\left\{1-L_{2} \cdot \exp \left(-L_{3}\left(E / I_{2 p}-1\right)\right)\right\} \\
\sigma_{2 s}^{I O N}(E / e V)\left[\mathrm{cm}^{2}\right]=q_{2 s} \cdot L_{4} \cdot \frac{\ln \left(E / I_{2 s}\right)}{E \cdot I_{2 s}} . \\
\cdot\left\{1-L_{5} \cdot \exp \left(-L_{6}\left(E / I_{2 s}-1\right)\right)\right\}
\end{gathered}
$$

The input parameters to Eq.(3) according to [57] are: $I_{2 p}=34 \mathrm{eV}$ and $I_{2 s}=67 \mathrm{eV}$, the binding energies of $2 \mathrm{p}$ respectively 2 s electrons, $q_{2 p}=6$ and $q_{2 s}=2$, the number of equivalent 2 p respectively $2 \mathrm{~s}$ electrons, and $L_{1}, \ldots, L 6$ are sets of individual constants for the $2 \mathrm{p}$ respectively $2 \mathrm{~s}$ subshell.

The overall formual for electron-impact target ionization now presents as

\begin{tabular}{|c|c|c||c|c|c|}
\hline \multicolumn{3}{|c||}{ 2p electrons } & \multicolumn{3}{c|}{ 2s electrons } \\
\hline$a_{2}$ & $b_{2}$ & $c_{2}$ & $a_{3}$ & $b_{3}$ & $c_{3}$ \\
\hline 3.49 & 0.81 & 0.087 & 10.8 & 0.46 & 1.5 \\
\hline
\end{tabular}

Parameters for the Lotz formula, Eq.(3. The values vary slidely from the ones presented by Lotz [56], but represent the experimental values excellently.

$$
\begin{gathered}
\sigma_{e^{-}}^{I O N}(E / e V)\left[\mathrm{cm}^{2}\right]= \\
=\left\{\begin{array}{l}
\sigma_{0}^{I O N} \quad \forall \quad E_{l o w} \leq E \leq I_{2 p} \\
\sigma_{0}^{I O N}+\sigma_{2 p}^{I O N} \quad \forall \quad I_{2 p} \leq E \leq I_{2 s} \\
\sigma_{0}^{I O N}+\sigma_{2 p}^{I O N}+\sigma_{2 s}^{I O N} \quad \forall \quad E \geq I_{2 s}
\end{array}\right.
\end{gathered}
$$

The constants in Eq.(3) have to be determined either by experiment, theory, or reasonable guesswork [57]. We were able to gather experimental data on ionization from the ground state in the energy region were inner-shell ionization occurs, see [33]. We now used Eq. (2 to fir ionization of the valence electron without considering inner-shell ionization. This led to the red line curve in Fig.2. Now the parameters $A_{1}, \ldots, A_{7}$ remained constant and we used

$\sigma_{\text {core }}^{I O N}(E / e V)\left[\mathrm{cm}^{2}\right]=\left\{\begin{array}{l}\sigma_{2 p}^{I O N} \quad \forall \quad I_{2 p} \leq E \leq I_{2 s} \\ \sigma_{2 p}^{I O N}+\sigma_{2 s}^{I O N} \quad \forall \quad E \geq I_{2 s}\end{array}\right.$

with variable parameters $L_{1}, \ldots, L_{6}$ to fit the inner-shell contribution.The obtained parameters, see Tab. D, vary slidely from the original parameters presented by Lotz [56], but represent the experimental values excellently. We therefore used these new t parameters, see Tab. D, to derive the core corrections in all ionization cross sections. In this way not only ionization including the core-correction but also pure valence ionization is represented for all transitions.

Fig.2 presents ground state electron impact ionization. It shows very clearly that the contributions from the core electrons are substantial in the high energy region and cannot be neglected. 


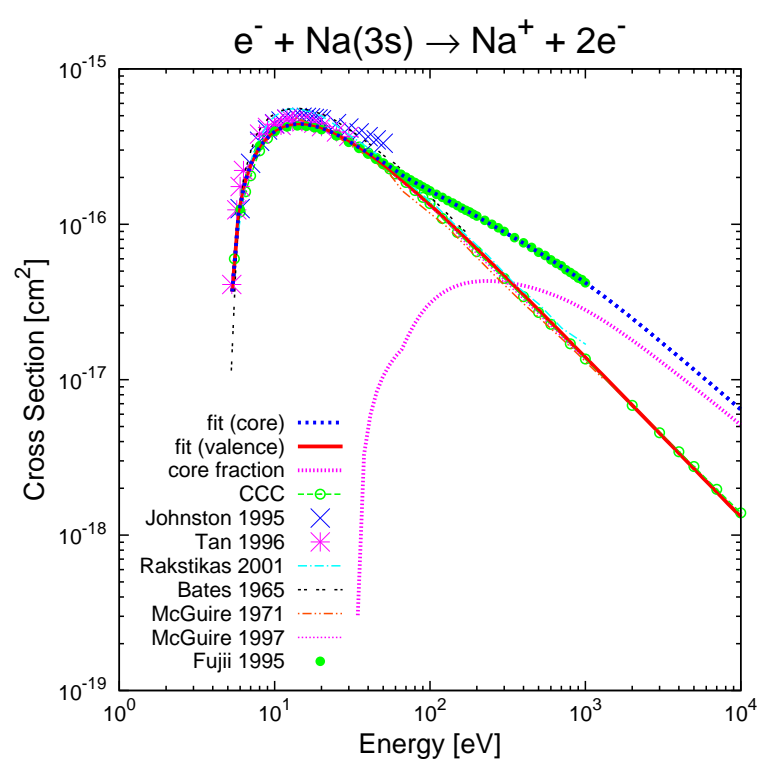

Fig. 2. Electron-impact target ionization from $\mathrm{Na}(3 \mathrm{~s})$. The fit of the valence is plotted according to Eq.(2) and Tab.2. The fit containing inner shell ionization ("fit with core") is plotted according to Eq.(4), where the core correction is added to Eq.(2) according to Eq.(3)) and Tab. D. The core fraction represents the contribution of inner-shell ionization according to Eq.(5) and Tab. D.

\subsection{Collisions with Protons}

If the velocities of relative motion are the same, and are sufficiently high, the cross sections for proton-atom collisions are equal to the cross sections for the corresponding electron-atom collision. The electron-impact cross sections need to be scaled according to the formula [58]

$$
\begin{gathered}
E_{H^{+}}[\mathrm{keV}]=0.9165 \cdot E[\mathrm{eV}] \\
\cdot\left(1-0.5 \cdot \frac{\Delta E}{E}+\sqrt{1-\frac{\Delta E}{E}}\right)
\end{gathered}
$$

This scaling is appropriate in the asymptotic region at high energies where Born approximation cross sections for $\mathrm{H}^{+}$and $\mathrm{e}^{-}$are the same at the same relative velocity of collision partners, since in this region the mechanism of direct excitation is the same for electrons and protons, see Fig.3. In the graphs depicting proton-impact target excitation cross sections, these scaled electron-impact cross sections are labeled "scaled CCC".

When the impact energy of the incident proton is lowered, a change from direct excitation to the forming of a quasi-molecule takes place. In this energy

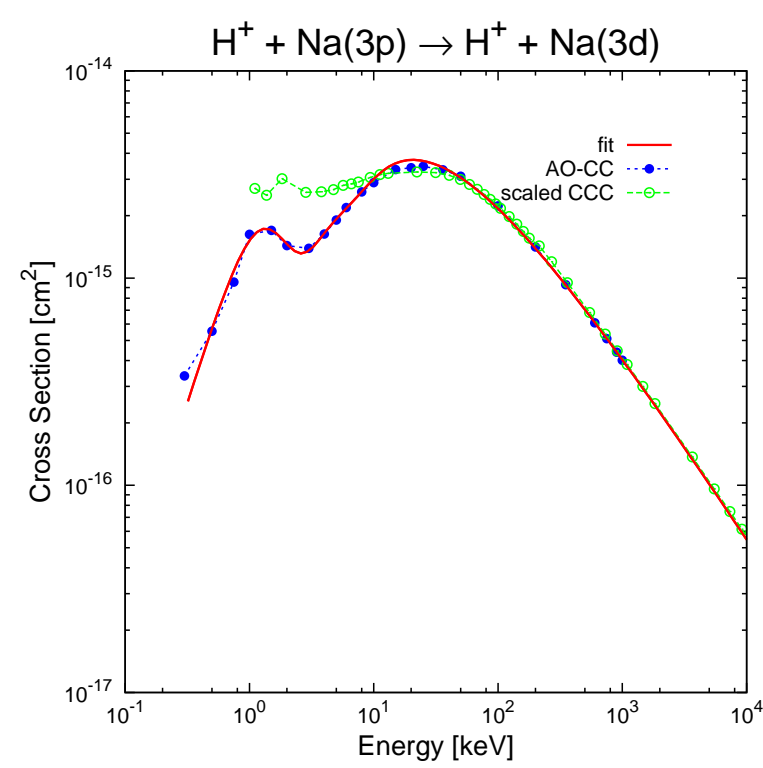

Fig. 3. Results from atomic-orbital close-coupling calculations for the $\mathrm{Na}(3 \mathrm{p} \rightarrow 3 \mathrm{~d})$ excitation process in collisions of $\mathrm{H}^{+}$with $\mathrm{Na}(3 \mathrm{p})$ in comparison with the corresponding fit (Eq.(7), Tab.3 \& 4) and with the scaled electron impact cross section. The latter has been derived from the new CCC calculations by applying the scaling relation from [58] (Eq.(6))

region the scaling relation (6) is no longer valid and single electron charge transfer becomes the dominant process. Originating from this interference of SEC and EXC channels the cross sections show oscillations. Due to this more complex behavior a formula with twelve fit parameters has to be used for proton-impact target excitation.

$$
\begin{gathered}
\sigma_{H^{+}}^{E X C}(E / k e V)\left[\mathrm{cm}^{2}\right]= \\
A_{1} \cdot 10^{-16}\left\{\frac{e^{-A_{2} / E} \cdot\left(A_{12}+\ln \left(A_{11}+A_{3} E\right)\right)}{E}+\right. \\
\left.+A_{4} \cdot \frac{e^{-A_{5} E}}{E^{A_{6}}}+A_{7} \cdot \frac{e^{-A_{8} / E}}{1+A_{9} \cdot E^{A_{10}}}\right\} \\
\forall E \geq E_{l o w}
\end{gathered}
$$

The fit parameters for EXC processes $\mathrm{Na}(\mathrm{nl} \rightarrow$ n'l') in collisions with $\mathrm{H}^{+}$are listed in Table $3 \& 4$. The theoretical data defining the fit for the $\mathrm{Na}(3 \mathrm{p}$ $\rightarrow 3 \mathrm{~d})$ EXC cross section are presented in Fig. 3 together with the fitted curve and the scaled electron- 
impact cross section derived from CCC results by using the scaling relation from [58] (Eq.(6)). The AO data from $\mathrm{Na}\left(\mathrm{nl} \rightarrow \mathrm{n}^{\prime} \mathrm{l}^{\prime}\right)$ EXC processes with $n=$ $3-5$ and $n^{\prime}=3-5$ are shown in Graphs in section 8.3 together with the fits and compared with experimental and other theoretical data where available and the scaled CCC cross sections; the comparison will be further discussed in the next section.

Proton-impact target electron loss (ELOSS) is the sum of proton-impact ionization and proton-impact single electron capture (SEC). There are no ELOSS data available in the literature. But there are data for both proton-impact SEC and ION. Fig.4-6 show, using the ground state $\mathrm{Na}(3 \mathrm{~s})$ as an example, how our new AO-CC calculations predict both SEC and ION cross sections excellently and how the SEC and ION fits if added together agree excellently with our AO-CC calculation of ELOSS. ION and SEC cross sections were fitted using the same formula.

$$
\begin{gathered}
\sigma_{H^{+}}^{I O N, S E C}(E / k e V)\left[\mathrm{cm}^{2}\right]= \\
=A_{1} \cdot 10^{-16}\left\{\frac{e^{-A_{2} / E} \cdot\left(A_{12}+\ln \left(A_{11}+A_{3} E\right)\right)}{E}+\right. \\
\left.+A_{4} \cdot \frac{e^{-A_{5} E}}{E^{A_{6}}}+A_{7} \cdot \frac{e^{-A_{8} / E}}{1+A_{9} \cdot E^{A_{10}}}\right\} \\
\forall E \geq E_{\text {low }}
\end{gathered}
$$

with the fit parameters $A_{1}-A_{12}$, tabulated in Tab.5, and $\mathrm{E}_{\text {low }}$ again the energy limit in the low energy region.

Finally the combined cross section, i.e. target electron loss, was fitted using the following formula:

$$
\begin{gathered}
\sigma_{H^{+}}^{E L O S S}(E / k e V)\left[\mathrm{cm}^{2}\right]=A_{1} \cdot 10^{-16} \\
\left\{\frac{e^{-A_{2} / E} \cdot \ln \left(1+A_{3} E\right)}{E}+A_{4} \cdot \frac{e^{-A_{5} E}}{E^{A_{6}}}\right\} \\
\forall E \geq E_{\text {low }}
\end{gathered}
$$

where $A_{1}-A_{6}$ are the fit parameters, see Tab.5, and $\mathrm{E}_{\text {low }}$ the limit of the validity of the fit in the low energy region.

The data for ELOSS involve processes with initial $\mathrm{Na}(\mathrm{nl}) \forall n \leq 5$. They have been compared with

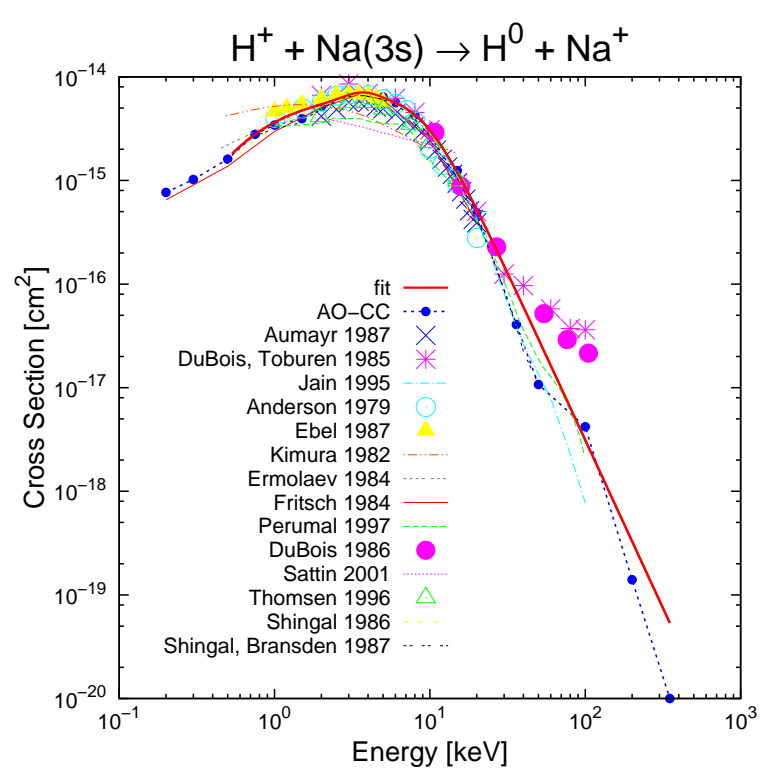

Fig. 4. Proton-impact single-electron capture cross sections from $\mathrm{Na}(3 \mathrm{~s})$. For references see Tab. F.

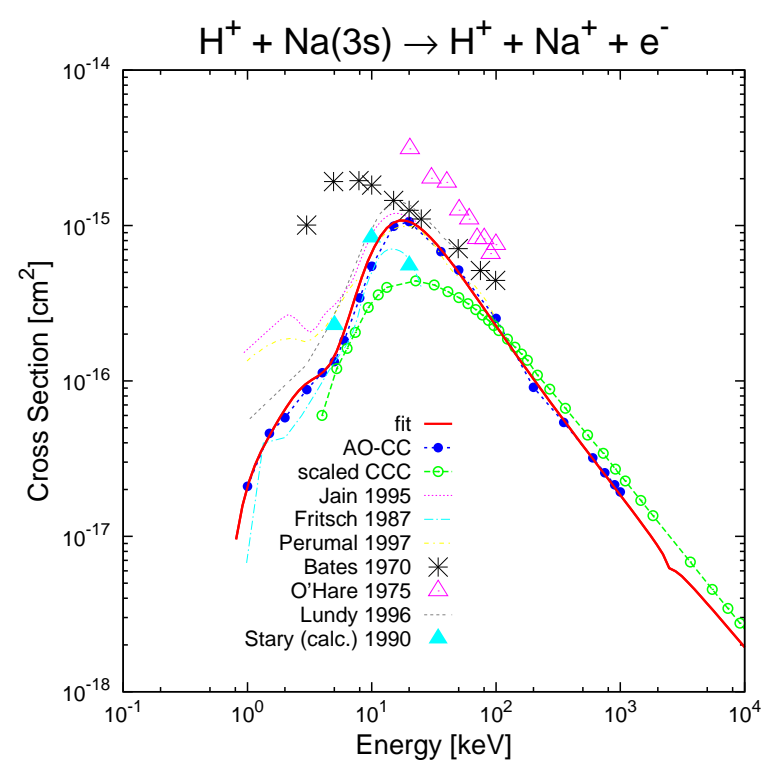

Fig. 5. Proton-impact target target ionization cross sections from $\mathrm{Na}(3 \mathrm{~s})$. For references see Tab. G.

scaled CCC calculation of electron-impact ionization cross sections. The agreement between these data is expected to be good in the energy region above $\approx 100 \mathrm{keV}$ where ionization is the dominant process in ELOSS. Unfortunately, the agreement is not always as good as hoped. This is due to the insufficient representation of ionization channels in the 


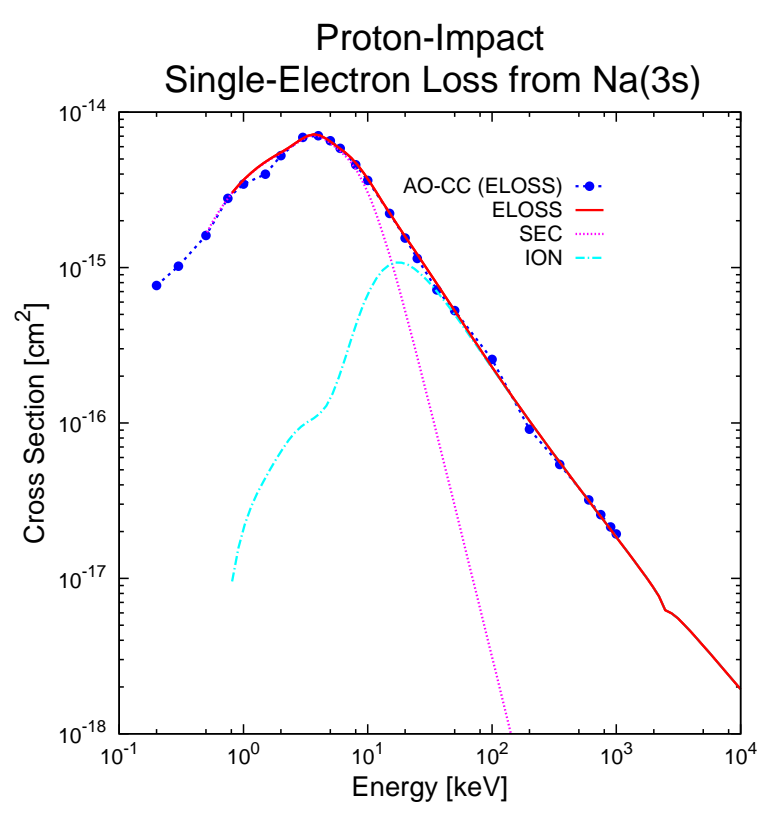

Fig. 6. Proton-impact target electron loss cross sections from $\mathrm{Na}(3 \mathrm{~s})$. AO-CC (ELOSS): present AO-CC calculations. ELOSS: fit for electron loss (Eq.(9)). SEC: fit for single-electron capture (Eq.(8)). ION: fit for ionization (Eq.(8))

AO-CC calculations. Furthermore the AO calculations neither include SEC from $\mathrm{Na}$ inner shells nor ionization of the latter.

\subsection{Scaling Relations for Collisions with Multiply Charged Ions}

A realistic fusion plasma is always polluted by a certain amount of impurity ions. Thus, to achieve satisfactory quality in simulations, it is necessary to include sodium - impurity ion collisions. The behavior of EXC cross sections with respect to a rescaling relation with projectile charge state $q$ and mass $m$ (in amu), derived from a three-state close-coupling dipole approximation [71] [72]. This scaling formula with respect to the electron binding energy $E_{b}$ of the initial state of the target sodium and the charge of the fully stripped projectile has been derived for single electron charge transfer from excited hydrogen atoms $\mathrm{H}(\mathrm{n})$ in a wide impact energy range. The same reduced impact energy and cross section are introduced for target excitation [17]

$$
E_{\text {red. }}^{\mathrm{EXC}}=\frac{E}{q}, \quad \sigma_{\text {red. }}^{\mathrm{EXC}}=\frac{\sigma}{q}
$$

and for single electron capture [17]

$$
E_{\text {red. }}^{\text {ELOSS }}=\frac{n^{2} \cdot E}{\sqrt{q}}, \quad \sigma_{\text {red. }}^{\text {ELOSS }}=\frac{\sigma}{n^{4} q}
$$

where $\mathrm{n}$ is the principal quantum number of the sodium target with respect to the binding energy $\left(n=\left(2 E_{b}\right)^{-1 / 2}\right)$ [17]. These well approved scaling relations have been applied to collisions involving $\mathrm{He}^{2+}$, and $\mathrm{Be}^{4+}$ projectiles. Fig.7 shows calculated excitation cross sections $(\mathrm{Na}(3 \mathrm{~s}) \rightarrow \mathrm{Na}(3 \mathrm{p}))$ by ion impact, whereas Fig.8 shows the corresponding reduced cross sections (in terms of Eq.(10)). In the energy region above reduced energies $\mathrm{E} / \mathrm{q}$ of about $3 \mathrm{keV} / \mathrm{amu}$, the fit of the proton-impact cross section (Eq.(7), Tab.3 \& 4) represents the other reduced cross sections well. A similar behavior can be observed for excitation from $\mathrm{Na}(3 \mathrm{p})$ to $\mathrm{Na}(3 \mathrm{~d})$ and to $\mathrm{Na}(4 \mathrm{~s})$, see Fig.9 and Fig.10, respectively. The qscaling typically breaks down below reduced energies $E / q<3 \mathrm{keV} / \mathrm{amu}$. In this low impact energy range no general behavior of EXC cross sections with respect to q can be expected.

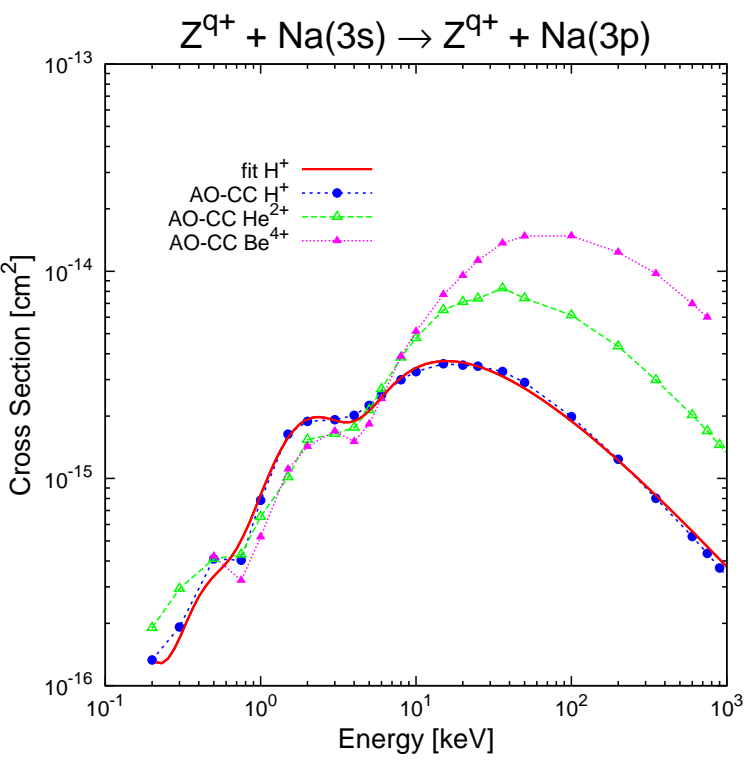

Fig. 7. Cross sections of collisions of $\mathrm{Na}$ with $\mathrm{H}^{+}, \mathrm{He}^{2+}$, and $\mathrm{Be}^{4+}$ ions for the transition $\mathrm{Na}(3 \mathrm{~s}) \rightarrow \mathrm{Na}(3 \mathrm{p})$. The fit is for proton-impact excitation in terms of Eq.(7) and Tab.3 \& 4

Fig.11 and 12 show reduced ELOSS cross sections from $\mathrm{Na}(\mathrm{ns})$ and $\mathrm{Na}(\mathrm{np})$ initial states, respectively. In the reduced energy region below $10 \mathrm{keV} / \mathrm{amu}$ reduced ELOSS cross sections from $\mathrm{Na}(\mathrm{ns})$ become almost independent from the energy of the incident particle, except for the $\mathrm{H}^{+}-\mathrm{Na}(3 \mathrm{~s})$ case (see Fig.11). The behavior of the latter is typical for a 


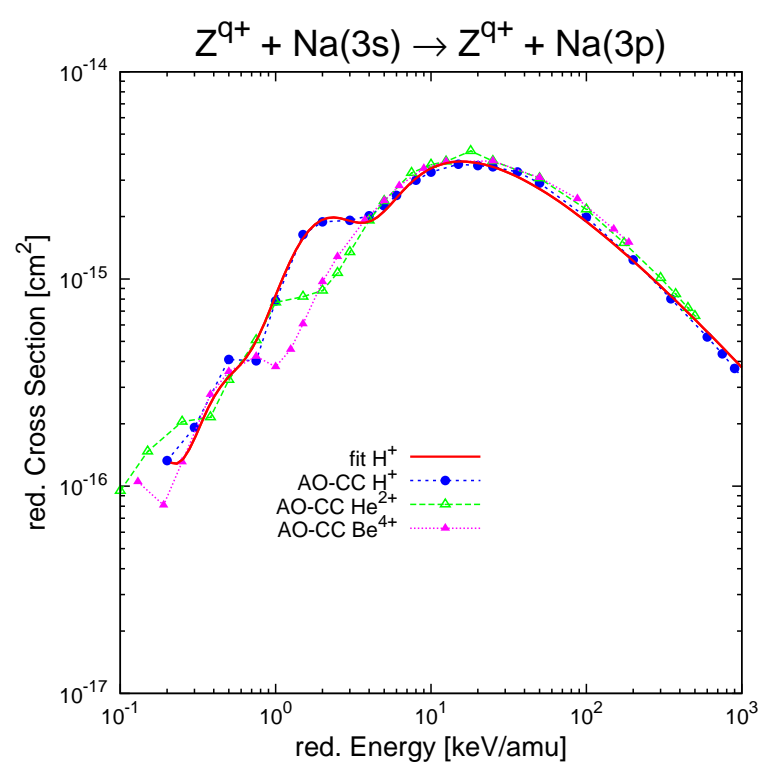

Fig. 8. Reduced cross sections $\sigma / q$ of collisions of $\mathrm{Na}_{\text {with }} \mathrm{H}^{+}$, $\mathrm{He}^{2+}$, and $\mathrm{Be}^{4+}$ ions for the transition $\mathrm{Na}(3 \mathrm{~s}) \rightarrow \mathrm{Na}(3 \mathrm{p})$. For reduced energies $E / q>3 \frac{\mathrm{keV}}{\mathrm{amu}}$, the reduced cross sections of $\mathrm{He}^{2+}$ and $\mathrm{Be}^{4+}$ follow the proton-impact cross section very well. The fit is for proton-impact excitation in terms of Eq.(7) and Tab.3 \& 4

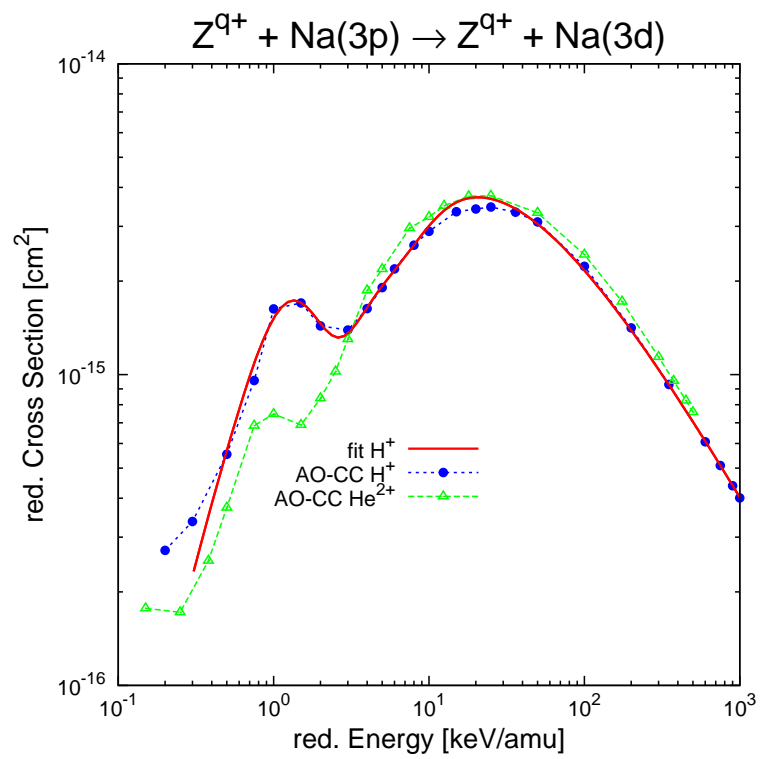

Fig. 9. Reduced cross sections $\sigma / q$ of collisions of $\mathrm{Na}$ with $\mathrm{H}^{+}$and $\mathrm{He}^{2+}$ ions for the transition $\mathrm{Na}(3 \mathrm{p}) \rightarrow \mathrm{Na}(3 \mathrm{~d})$. The fit is for proton-impact excitation in terms of Eq.(7) and Tab.3 \& 4

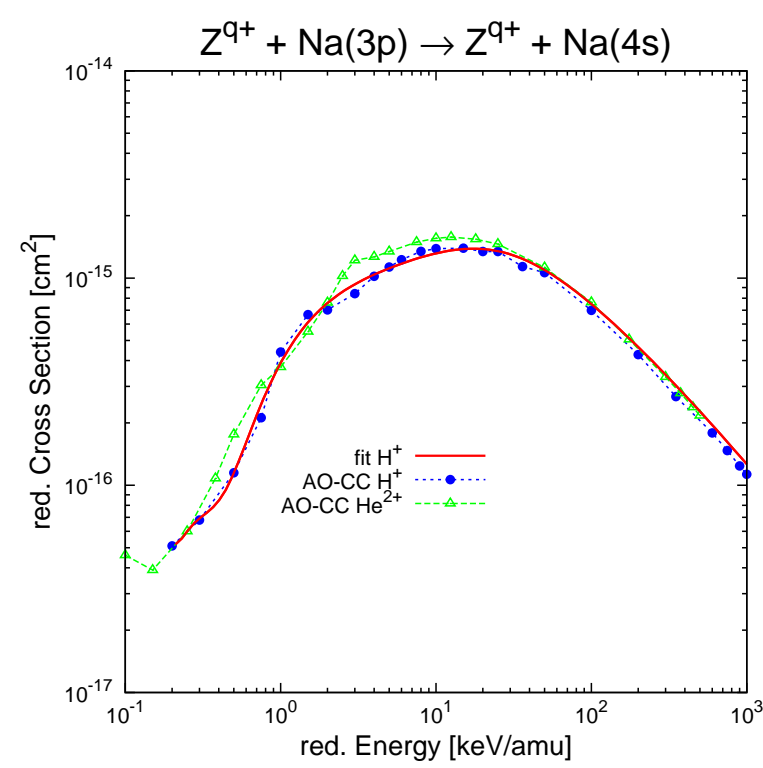

Fig. 10. Reduced cross sections $\sigma / q$ of collisions of $\mathrm{Na}$ with $\mathrm{H}^{+}, \mathrm{He}^{2+}$, ions for the transition $\mathrm{Na}(3 \mathrm{p}) \rightarrow \mathrm{Na}(4 \mathrm{~s})$. The fit is for proton-impact excitation in terms of Eq.(7) and Tab.3 \& 4

non-resonant SEC, whereas all other collision systems are close to resonance $\left(\mathrm{H}^{+}-\mathrm{Na}(4 \mathrm{~s}, 5 \mathrm{~s})\right)$ or are quasi-resonant $\left(\mathrm{Z}^{q+}-\mathrm{Na}(\mathrm{ns})\right)$. Therefore the fitted (Eq.(9)) reduced $\mathrm{He}^{2+}$ ELOSS cross section represents a general curve from which cross sections for any projectile of higher $\mathrm{q}$ and initial $\mathrm{Na}(\mathrm{ns})$ can be derived. In the case of initial $\mathrm{Na}(\mathrm{np})$ the situation is similar and again the fit to the $\mathrm{He}^{2+}$ data serve as the general curve from which all cross sections for higher charged projectiles can be derived. Tab. 8 shows the corresponding fit parameters. For even higher excited initial $\mathrm{Na}(\mathrm{nl})$ the scaling relation (Eq.(11)) can be directly applied to the fit of $\mathrm{H}^{+}$$\mathrm{Na}(\mathrm{nl})$. 


\begin{tabular}{|c|c|c|c|c|c|}
\hline \multicolumn{6}{|c|}{ Proton-impact target excitation } \\
\hline Transition & Name & Published in & Referenced in & Weight in fit & Comment \\
\hline \multirow[t]{11}{*}{$3 \mathrm{~s} \rightarrow 3 \mathrm{p}$} & Allen 1988 & {$[40]$} & [41] & Very high & Experiment \\
\hline & Aumayr 1987 & {$[42]$} & - & Very high & Experiment \\
\hline & Fritsch 1987 & {$[43]$} & {$[44]$} & High & Calculation \\
\hline & Howald 1983 & {$[45]$} & - & Low & Experiment \\
\hline & Jain 1995 & {$[46]$} & - & High & Calculation \\
\hline & Jitschin 1986 & {$[47]$} & - & Low & Experiment \\
\hline & Lavrov 1983 & {$[48]$} & {$[49]$} & Low & Experiment \\
\hline & Shingal (AO) 1986 & {$[50]$} & - & High & Atomic-orbital calculation \\
\hline & Shingal, Bransden 1987 & {$[44]$} & - & Low & Calculation \\
\hline & Stary 1990 & {$[51]$} & - & Moderate & Calculation \\
\hline & Theodosiou 1987 & {$[49]$} & - & Very low & Calculation \\
\hline \multirow[t]{5}{*}{$3 \mathrm{~s} \rightarrow 3 \mathrm{~d}$} & Anderson 1979 & {$[52]$} & - & Moderate & Experiment \\
\hline & Anderson 1985 & {$[53]$} & - & Moderate & Experiment \\
\hline & Jain 1995 & {$[46]$} & - & High & Calculation \\
\hline & Shingal, Bransden 1987 & {$[44]$} & - & High & Calculation \\
\hline & Theodosiou 1987 & {$[49]$} & - & Low & Calculation \\
\hline $3 \mathrm{~s} \rightarrow 4 \mathrm{~s}$ & Theodosiou 1987 & {$[49]$} & - & Low & Calculation \\
\hline $3 \mathrm{~s} \rightarrow 4 \mathrm{p}$ & Theodosiou 1987 & {$[49]$} & - & Low & Calculation \\
\hline $3 \mathrm{~s} \rightarrow 4 \mathrm{~d}$ & Theodosiou 1987 & {$[49]$} & - & Low & Calculation \\
\hline $3 \mathrm{~s} \rightarrow 4 \mathrm{f}$ & Theodosiou 1987 & [49] & - & Low & Calculation \\
\hline
\end{tabular}

Table C

Used references for proton-impact target excitation. For description of column contents see Table A.

Table E

\begin{tabular}{|c|c|c|c|c|c|c|c|c|c|c|c|}
\hline & $\mathbf{E}_{\text {low }}$ & $\mathbf{A}_{1}$ & $\mathbf{A}_{2}$ & $\mathbf{A}_{3}$ & $\mathbf{A}_{4}$ & $\mathbf{A}_{5}$ & $\mathbf{A}_{6}$ & $\mathbf{A}_{7}$ & $\mathbf{A}_{8}$ & $\mathbf{A}_{9}$ & $\begin{array}{lll}\mathbf{A}_{10} & \mathbf{A}_{11} & \mathbf{A}_{12}\end{array}$ \\
\hline ION & $\mid 1$ & 8.389 & $8.83-7$ & $-7.95 \cdot 10^{-4}$ & -0.179 & 0.4266 & -2.554 & 1.13 & 2.095 & $1.52 \cdot 10^{-3}$ & 1.9982 .0221 .21 \\
\hline SEC & 0.5 & 24.21 & 1.0 & 0 & $1.68 \cdot 10^{-3}$ & 5.02 & -18.34 & 3.342 & 0.7988 & $8.155 \cdot 10^{-4}$ & $3.25 \quad 1.0$ \\
\hline
\end{tabular}

Fit parameters for proton-impact target ionization (ION) and single-electron capture (SEC) from $\mathrm{Na}(3 \mathrm{~s})$ 
Table F

\begin{tabular}{|c|c|c|c|c|l|}
\hline \multicolumn{6}{|c|}{ Proton-impact single-electron capture } \\
\hline Ground state & Name & Published in & Referenced in & Weight in fit & Comment \\
\hline 3 3s & Anderson 1979 & {$[52]$} & {$[46]$} & High & Experiment \\
& Aumayr 1987 & {$[42]$} & - & Very high & Experiment \\
& DuBois, Toburen 1985 & {$[59]$} & - & Very high & Experiment \\
& DuBois 1986 & {$[60]$} & - & Moderate & Experiment \\
& Ebel 1987 & {$[61]$} & - & Moderate & Experiment \\
& Ermolaev 1984 & {$[62]$} & {$[61]$} & Moderate & Calculation \\
& Fritsch 1984 & {$[63]$} & - & Very high & Calculation \\
& Jain 1995 & {$[46]$} & - & Very high & Calculation \\
& Kimura 1982 & {$[64]$} & {$[61]$} & Low & Calculation \\
& Permual 1997 & {$[65]$} & - & Moderate & Calculation \\
& Sattin 2001 & {$[66]$} & - & Very low & Calculation \\
& Shingal 1986 & {$[50]$} & - & High & Calculation \\
& Shingal, Bransden 1987 & {$[44]$} & - & High & Calculation \\
& Thomsen 1996 & {$[67]$} & {$[66]$} & High & Experiment \\
\hline
\end{tabular}

Used references for proton-impact single-electron capture cross sections. For description of column contens see Table A

\begin{tabular}{|c|c|c|c|c|c|}
\hline \multicolumn{7}{|c|}{ Proton-impact target ionization } \\
\hline Ground state & Name & Published in & Referenced in & Weight in fit & Comment \\
\hline 3s & Bates 1970 & {$[68]$} & {$[65]$} & Low & Experiment \\
& Fritsch 1987 & {$[43]$} & {$[46]$} & High & Calculation \\
& Jain 1995 & {$[46]$} & - & Moderate & Calculation \\
& Lundy 1996 & {$[69]$} & - & Moderate & Calculation \\
& O'Hare 1975 & {$[70]$} & - & Low & Experiment \\
& Perumal 1997 & {$[65]$} & - & Moderate & Calculation \\
& Stary (calc.) 1990 & {$[51]$} & - & Low & Optical potential calculations \\
\hline
\end{tabular}

Table G

Used references for proton-impact target ionization cross sections. For description of column contents see Table A 


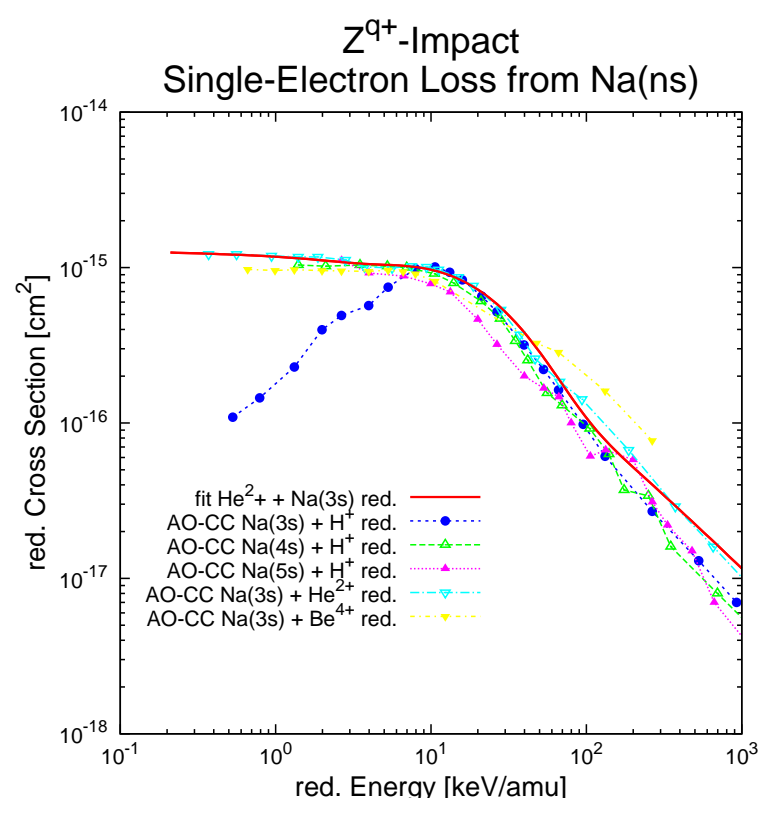

Fig. 11. Reduced cross sections of collisions of $\mathrm{Na}(\mathrm{ns})$ with $\mathrm{H}^{+}, \mathrm{He}^{2+}$, and $\mathrm{Be}^{4+}$ ions resulting in Na electron loss. Eq.(9) was fitted to the reduced $\mathrm{He}^{2+}$ cross section. For parameters see Tab.8.

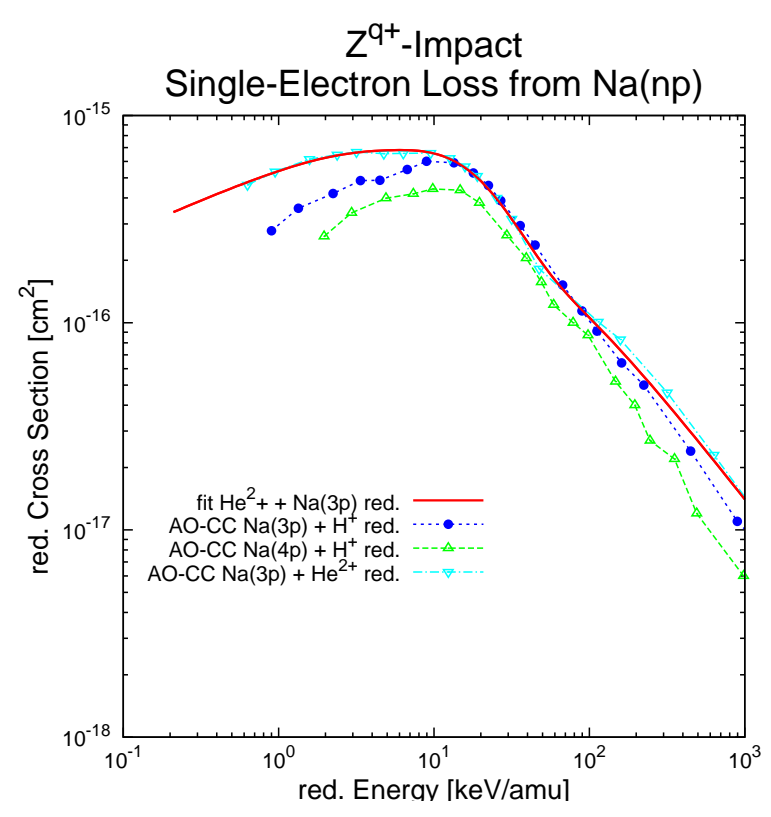

Fig. 12. Reduced cross sections of collisions of $\mathrm{Na}(\mathrm{np})$ with $\mathrm{H}^{+}$and $\mathrm{He}^{2+}$ ions resulting in Na electron loss. Eq.(9) was fitted to the reduced $\mathrm{He}^{2+}$ cross section. For parameters see Tab.8.

\begin{tabular}{|c||c||c|c|c|c|c|c|}
\hline $\mathbf{n l}$ & $\mathbf{E}_{\text {low }}$ & $\mathbf{A}_{\mathbf{1}}$ & $\mathbf{A}_{\mathbf{2}}$ & $\mathbf{A}_{\mathbf{3}}$ & $\mathbf{A}_{\mathbf{4}}$ & $\mathbf{A}_{\mathbf{5}}$ & $\mathbf{A}_{\mathbf{6}}$ \\
\hline $3 \mathrm{~s}$ & 0.2 & 3.70587 & 15.8977 & $-6.4856 \cdot 10^{10}$ & 3.31056 & 0.04344 & 0.01715 \\
$3 \mathrm{p}$ & 0.2 & 4.5237 & 23.7408 & $-6.4855 \cdot 10^{10}$ & 1.2994 & 0.08556 & -0.33615 \\
\hline
\end{tabular}

Fit parameters for reduced cross sections of $\mathrm{He}^{2+}$ impact of $\mathrm{Na}(\mathrm{nl}) ; n=3$.

\section{Acknowledgements}

This work, supported by the European Communities under the contract of Association between EURATOM and the Austrian Academy of Sciences, was carried out within the framework of the European Fusion Development Agreement. The views and opinions expressed herein do not necessarily reflect those of the European Commission.

$\mathrm{KI}$ is a recipient of a DOC-fFORTE-fellowship of the Austrian Academy of Sciences at Institut für Allgemeine Physik at Vienna University of Technology.

This work was also supported by the FriedrichSchiedel-Stiftung für Energietechnik.

The work of IB was supported by the Australian Research Council, the Australian Partnership for Advanced Computing and its Western Australian node iVEC.

\section{References}

[1] F. Aumayr, R. Schorn, M. Pöckl, J. Schweinzer, E. Wolfrum, K. McCormick, E. Hintz, H. Winter, Tokamak edge plasma densities measured by means of active Lithium beam diagnostics, J. Nucl. Mat. 196-198 (1992) 928-932.

[2] J. Schweinzer, E. Wolfrum, F. Aumayr, M. Pöckl, H. Winter, R. Schorn, E. Hintz, A. Unterreiter, Reconstruction of plasma edge density profiles from LiI $(2 \mathrm{~s}-2 \mathrm{p})$ emission profiles, Plasma Physics and Controlled Fusion 34 (1992) 1173-1183.

[3] E. Wolfrum, F. Aumayr, D. Wutte, H. Winter, E. Hintz, D. Rusbüldt, R. Schorn, Fast lithium - beam spectroscopy of tokamak edge plasmas, Rev. Sc. Instr. 64 (1993) 2285-2292.

[4] R. Brandenburg, J. Schweinzer, S. Fiedler, F. Aumayr, H. Winter, Modelling of fast neutral Li beams for fusion edge plasma diagnostics, Plasma Physics and Controlled Fusion 41 (1999) 471-484.

[5] F.

Aumayr,

H. Winter, Plasmadiagnostik mit Lithiumatomstrahl aktivierter Umladungsspektroskopie, Ann. d. Physik 42 (1985) 228-238.

[6] D. Wutte, R. Janev, F. Aumayr, M. Schneider, J. Schweinzer, J. Smith, H. Winter, Cross Sections for Collision Processes of Li Atoms Interacting With Electrons, Protons, Multiply Charged Ions, And Hydrogen Molecules, Atomic Data \& Nuclear Data Tables 65 (1997) 155-180, article No. DT970736. 
[7] J. Schweinzer, R. Brandenburg, I. Bray, R. Hoekstra, F. Aumayr, R. Janev, H. Winter, Database for Inelastic Collisions of Lithium Atoms With Electrons, Protons, And Multiply Charged Ions, Atomic Data \& Nuclear Data Tables 72 (1999) 239-273, article ID adnd. 1999.0815.

[8] S. Fiedler, R. Brandenburg, J. Baldzuhn, K. McCormick, F. Aumayr, J. Schweinzer, H. Winter, W. . A. U. team, Edge Plasma Diagnostics on W7-AS and ASDEX Upgrade using fast Li Beams, J. Nucl. Mat. 266-269 (1999) 1279-1284.

[9] R. Schorn, E. Hintz, D. Rusbüldt, F. Aumayr, M. Schneider, E. Unterreiter, H. Winter, Absolute concentrations of light impurity ions in tokamak discharges measured with Lithium beam-activated charge exchange spectroscopy, J. Appl. Phys. B 52 (1991) 71-78.

[10] R. Schorn, E. Wolfrum, F. Aumayr, E. Hintz, D. Rusbüldt, H. Winter, Radial temperature distributions of $\mathrm{C} 6+-$ ions in the TEXTOR edge plasma measured with Li-CXS, Nuclear Fusion 32 (1992) 351-359.

[11] E. Wolfrum, J. Schweinzer, M. Reich, L. Horton, C. Maggi, Impurity ion density measurements at the plasma edge of ASDEX Upgrade using lithium beam charge exchange spectroscopy, Rev. Sc. Instr. 77 (2006) 033507.

[12] I. Bray, A. T. Stelbovics, Convergent close-coupling calculations of electron-hydrogen scattering, Phys. Rev. A 46 (11) (1992) 6995-7011.

[13] I. Bray, Convergent close-coupling method for the calculation of electron scattering on hydrogen-like targets, Phys. Rev. A 49 (1994) 1066-1082.

[14] I. Bray, A. Stelbovics, Explicit Demonstration of the Convergence of the Close-Coupling Method for a Coulomb Three-Body Problem, Phys. Rev. Lett. 69 (1) (1992) 53-56.

[15] R. Brandenburg, J. Schweinzer, F. Aumayr, H. Winter, $\operatorname{Li}(2 \mathrm{p} \leftarrow 2 \mathrm{~s})$ excitation by impact of slow ions, J. Phys. B: At. Mol. Phys. 31 (1998) 2585-2599.

[16] W. Fritsch, C. Lin, The semiclassical close-coupling description of atomic collisions: recent developments and results, Physics Reports - A Review Section of Physics Letters $202(1-2)(1991) 1-97$.

[17] J. Schweinzer, D. Wutte, H. P. Winter, A study of electron capture and excitation processes in collisions of multiply charged ions with lithium atoms, J. Phys. B: At. Mol. Phys. 27 (1994) 137-153.

[18] S. Buckman, P. Teubner, Differential cross sections for the electron impact excitation of the $3^{2} \mathrm{P}_{1 / 2,3 / 2}$ resonance levels of sodium, J. Phys. B: At. Mol. Phys. 12 (1979) 1741-1750.

[19] J. Mitroy, I. McCarthy, A. Selbovics, Electron scattering from sodium at intermediate energies, J. Phys. B: At. Mol. Phys. 20 (1987) 4827-4850.

[20] E. Enemark, A. Gallagher, Electron Excitation of the Sodium D Lines, Phys. Rev. A 6 (1) (1972) 192-205.

[21] G. Gould, Atomic beam measurements of some sodium excitation functions, Ph.D. thesis, University of New South Wales (1970).

[22] D. Moores, D. Norcross, The scattering of electrons by sodium atoms, J. Phys. B: At. Mol. Phys. 5 (1972) 1482-
1505.

[23] E. Karule, Polarization of lithium and sodium atom resonance lines excited by electron impact, J. Phys. B: At. Mol. Phys. 3 (1970) 860-864.

[24] Y.-K. Kim, Scaling of plane-wave Born cross sections for electron-impact excitation of neutral atoms, Phys. Rev. A 64 (3) (2001) 032713.

[25] J. Phelps, C. Lin, Electron-impact excitation of the sodium atom, Phys. Rev. A 24 (3) (1981) 1299-1326.

[26] S. Srivastava, L. Vušković, Elastic and inelastic scattering of electrons by Na, J. Phys. B: At. Mol. Phys. 13 (1980) 2633-2643.

[27] I. Zapesochnyi, E. Postoi, I. Aleksakhin, Sov. Phys. JETP 41 (1976) 865.

[28] P. Ganas, Excitation of sodium atoms by electron impact, J. Appl. Phys. 57 (1) (1985) 154-156.

[29] B. Stumpf, A. Gallagher, Electron excitation of $\mathrm{Na}(3 \mathrm{~S})$ and $\mathrm{Na}(3 \mathrm{P})$ atoms to the $\mathrm{Na}(3 \mathrm{D})$ state, Phys. Rev. A 32 (6) (1985) 3344-3353.

[30] S. Verma, R. Srivastava, Electron impact excitation of the $3^{2} \mathrm{~d}$ states of lithium, sodium and potassium atoms, J. Phys. B: At. Mol. Phys. 29 (1996) 3215-3234.

[31] D. Bates, A. Boyd, S. Prasad, Impact ionization of sodium, Proc. Phys. Soc. London 85 (1965) 1121-1126.

[32] K. Omidvar, H. Kyle, E. Sullivan, Ionization of Multielectron Atoms by Fast Charged Particles, Phys. Rev. A 5 (3) (1972) 1174-1187.

[33] K. Fujii, S. Srivastava, A measurement of electronimpact ionization cross section of sodium, J. Phys. B: At. Mol. Phys. 28 (1995) L559-563.

[34] A. Johnston, P. Burrow, Electron-impact ionization of Na, Phys. Rev. A 51 (3) (1995) R1735-R1737.

[35] E. McGuire, Inelastic Scattering of Electrons and Protons by the Elements He to Na, Phys. Rev. A 3 (1) (1971) 267-279.

[36] E. McGuire, Systematics of ns subshell electron ionization cross sections, J. Phys. B: At. Mol. Phys. 30 (1997) 1563-1587.

[37] N. Rakštikas, A. Kupliauskienè, The Influence of Valence Electron State on the 2p Ionization of Atomic Sodium by Electrons, Physica Scripta 64 (2001) 230-236.

[38] W. S. Tan, Z. Shi, C. H. Ying, L. Vušković, Electronimpact ionization of laser-excited sodium atom, Phys. Rev. A 54 (5) (1996) R3710-R3713.

[39] I. Zapesochnyi, I. Aleksakhin, Ionization of alkali-metal atoms by slow electrons, Zh. Eksp. Teor. Fiz. 55 (1968) $76-85$.

[40] J. Allen, L. Anderson, C. Lin, Cross section for excitation of sodium by impact of $\mathrm{H}^{+}, \mathrm{H}_{2}^{+}, \mathrm{H}_{3}^{+}$, and $\mathrm{H}^{-}$ ions, Phys. Rev. A 37 (2) (1988) 349-355.

[41] C. Theodosiou, Theoretical verification of the differences between excitation of $\mathrm{Na} 3 \mathrm{p}$ and $\mathrm{Na} 3 \mathrm{~d} \mathrm{by} \mathrm{H}^{+}$and $\mathrm{H}^{-}$ impact, Phys. Rev. A 38 (9) (1988) 4923-4926.

[42] F. Aumayr, G. Lakits, H. Winter, Charge transfer and target excitation in $\mathrm{H}^{+}-\mathrm{Na}(3 \mathrm{~s})$ collisions $(2-20 \mathrm{keV})$, J. Phys. B: At. Mol. Phys. 20 (1987) 2025-2030.

[43] W. Fritsch, Theoretical study of Na excitation and ionization in $\mathrm{H}^{+}+\mathrm{Na}$ collisions, Phys. Rev. A 35 (5) (1987) 2342-2344.

[44] R. Shingal, B. Bransden, Charge transfer, target excitation and ionisation in $\mathrm{H}^{+}+\mathrm{Na}(3 \mathrm{~s})$ collisions, $\mathrm{J}$. Phys. B: At. Mol. Phys. 20 (1987) 4815-4825. 
[45] A. Howald, L. Anderson, C. Lin, Excitation of the $\mathrm{Na}(3 \mathrm{p})$ Level by $\mathrm{H}^{-}$Ions and $\mathrm{H}^{0}$ Atoms, Phys. Rev. Lett. 51 (22) (1983) 2029-2032.

[46] A. Jain, T. Winter, Electron transfer, target excitation, and ionization in $\mathrm{H}^{+}+\mathrm{Na}(3 \mathrm{~s})$ and $\mathrm{H}^{+}+\mathrm{Na}(3 \mathrm{p})$ collisions in the coupled-Sturmian-pseudostate approach, Phys. Rev. A 51 (4) (1995) 2963-2973.

[47] W. Jitschin, S. Osimitsch, D. Mueller, H. Reihl, R.J.Allan, O. Schöller, H. Lutz, Excitation of the Na 3p state by proton impact, J. Phys. B: At. Mol. Phys. 19 (1986) 2299-2309.

[48] V. Lavrov, R. Lomsadze, Abstracts of Contributed Papers, Thirteenth Interational Conference on the Physics of Electronic and Atomics Collisions, Berlin, North-Holland, Amsterdam, 1983, p. 610.

[49] C. Theodosiou, Excitation of the Na 3p level by light ions, Phys. Rev. A 36 (5) (1987) 2067-2071.

[50] R. Shingal, B. Bransden, A. Ermolaev, D. Flower, C. Newby, C. Noble, Charge transfer in $\mathrm{H}^{+}-\mathrm{Na}^{0}$ collisions: atomic orbital calculations, J. Phys. B: At. Mol. Phys. 19 (1986) $309-320$.

[51] C. Stary, H. Lüdde, R. Dreizler, Optical potential description of collisions of $\mathrm{p}$ and $\overline{\mathrm{p}}$ with alkali atoms, J. Phys. B: At. Mol. Phys. 23 (1990) 263-277.

[52] C. Anderson, A. Howald, L. Anderson, Charge transfer cross sections and equilibrium fractions for $1-25 \mathrm{keV}$ $\mathrm{H}^{+}$ions incident of a Na vapor target, Nucl. Instrum. Meth. 165 (3) (1979) 583-587.

[53] L. Anderson, J. Allen, C. Lin, R. Miers, in: M. C. et al. (Ed.), Proc. 14th Int. Conf. on Physics of Electronic and Atomic Collisions, North Holland, Amsterdam, 1985, p. 385.

[54] W. H. Press, Numerical recipes in C - the art of scientific computing, Cambridge University Press, 1992, Ch. 15.5, pp. 681-688.

[55] T. Williams, C. Kelley, gnuplot - An Interactive Plotting Program (2004).

[56] W. Lotz, Electron-Impact Ionization Cross-Sections and Ionization Rate Coefficients for Atoms and Ions from Hydrogen to Calcium, Zeitschrift für Physik 216 (1968) 241-247.

[57] W. Lotz, Subshell Binding Energies of Atoms and Ions from Hydrogen to Zinc, J. Opt. Soc. Am. 58 (7) (1968) 915-921.

[58] D. Bates, G. Griffing, Inelastic Collisions between Heavy Particles I: Excitation and Ionization of Hydrogen Atoms in Fast Encounters with Protons and with other Hydrogen Atoms, Proc. Phys. Soc. 66 (1953) 961-971.

[59] R. DuBois, L. Toburen, Electron capture by protons and helium ions from lithium, sodium, and magnesium, Phys. Rev. A 31 (6) (1985) 3603-3611.

[60] R. DuBois, Charge transfer leading to multiple ionization of neon, sodium, and magnesium, Phys. Rev. A 34 (4) (1986) 2738-2745.

[61] F. Ebel, E. Salzborn, Charge transfer of $0.2-5.0 \mathrm{keV}$ protons and hydrogen atoms in sodium-, potassium- and rubidium-vapour targets, J. Phys. B: At. Mol. Phys. 20 (1987) 4531-4542.

[62] A. Ermolaev, Charge transfer in collisions between protons and lithium atoms, J. Phys. B: At. Mol. Phys. 17 (6) (1984) 1069-1082.
[63] W. Fritsch, Atomic-basis study of electron transfer in $\mathrm{H}^{+}+\mathrm{Na}$ and $\mathrm{H}^{+}+\mathrm{K}$ collisions, Phys. Rev. A 30 (2) (1984) 1135-1138.

[64] M. Kimura, R. Olson, J. Pascale, Molecular treatment of electron capture by protons from ground and excited states of alkali-metal atoms, Phys. Rev. A 26 (6) (1982) 3113-3124.

[65] A. Perumal, D. Tripathi, Charge Transfer and Ionization in Proton-Alkali Atoms Collisions with and without Electric Field, J. Phys. Soc. of Japan 66 (12) (1997) 3783-3789.

[66] F. Sattin, Further study of the over-barrier model to compute charge-exchange processes, Phys. Rev. A 64 (3) (2001) 034704.

[67] J. W. Thomsen, N. Andersen, D. Dowek, J. C. Houver, J. H. V. Lauritsen, U. Müller, J. O. P. Pedersen, J. Salgado, A. Svensson, Orbital alignment dependence of electron transfer cross sections. IV: $1-15 \mathrm{keV} \mathrm{Ne+,}$ Ar+-Na (3p) collisions, Z. Phys. D: At., Mol. Clusters 37 (2) (1996) 133-139.

[68] D. Bates, A. Kingston, Vol. 6, Academic Press New York, 1970, p. 269.

[69] C. Lundy, R. Olson, A classical analysis of proton collisions with ground-state and excited, aligned sodium targets, J. Phys. B: At. Mol. Phys. 29 (1996) 1723-1736.

[70] B. O'Hare, R. McCullough, H. Gilbody, Ionization of sodium and potassium vapour by $20-100 \mathrm{keV} \mathrm{H}^{+}$and $\mathrm{He}^{+}$ions, J. Phys. B: At. Mol. Phys. 8 (18) (1975) 29682978.

[71] R. Janev, L. Presnyakov, Single-electron excitation and ionisation processes in atom-multicharged-ion collisions, J. Phys. B: At. Mol. Phys. 13 (1980) 4233-4244.

[72] R. Janev, Unified cross section scaling for electron capture from excited hydrogen atoms by multicharged ions, Physics Letters A 160 (1991) 67-70. 
5. Explanation of Tables

Table 1: $\quad$ Fit Parameters for Electron-Impact Target-Excitation Cross Sections of $\mathrm{Na}\left(\mathrm{nl} \rightarrow \mathbf{n}^{\prime} \mathbf{l}^{\prime}\right) ; \mathbf{n}, \mathbf{n}^{\prime}=\mathbf{3}-\mathbf{5}$

$n l \rightarrow n^{\prime} l^{\prime} \quad$ Initial - final states of the $\mathrm{Na}$ atom

$\Delta \mathrm{E} \quad$ Excitation energy of the EXC process in $\mathrm{eV}$

$\mathrm{A}_{1}, \ldots, \mathrm{A}_{7}$ Fit parameters as in Eq.(1)

Table 2: $\quad$ Fit Parameters for Electron-Impact Target-Ionization Cross Sections

$$
\begin{array}{ll}
\text { of } \mathbf{N a}(\mathbf{n l}) ; \mathbf{n}=\mathbf{3}-\mathbf{5} \\
n l & \text { Initial state of the Na atom } \\
\mathrm{I}_{n l} & \text { Ionization energy in } \mathrm{eV} \\
\mathrm{A}_{1}, \ldots, \mathrm{A}_{6} & \text { Fit parameters as in Eq. }(2)
\end{array}
$$

Tables 3 \& 4: Fit Parameters for Proton-Impact Target-Excitation Cross Sections

$$
\begin{aligned}
& \text { of } \mathbf{N a}\left(\mathbf{n l} \rightarrow \mathbf{n}^{\prime} \mathbf{l}^{\prime}\right) ; \mathbf{n}, \mathbf{n}^{\prime}=\mathbf{3}-\mathbf{5} \\
& n l \rightarrow n^{\prime} l^{\prime} \quad \text { Initial - final states of the Na atom } \\
& \mathrm{A}_{1}, \ldots, \mathrm{A}_{12} \text { Fit parameters as in Eq. }(7)
\end{aligned}
$$

Table 5: $\quad$ Fit Parameters for Proton-Impact Target-Electron-Loss Cross Sections of $\mathrm{Na}(\mathrm{nl}) ; \mathbf{n}=\mathbf{3}-\mathbf{5}$

nl Initial state of the $\mathrm{Na}$ atom

$\mathrm{A}_{1}, \ldots, \mathrm{A}_{6}$ Fit parameters as in Eq.(9)

6. Explanation of Graphs

Section 8.1: Electron-impact target-excitation cross sections of $\mathrm{Na}\left(\mathbf{n l} \rightarrow \mathbf{n}^{\prime} \mathbf{l}^{\prime}\right) ; \mathbf{n}, \mathbf{n}^{\prime}=\mathbf{3}-\mathbf{5}$ $n l \rightarrow n^{\prime} l^{\prime}$ Initial - final states of the $\mathrm{Na}$ atom

Section 8.2: Electron-impact target-ionization cross sections from $\mathrm{Na}(\mathrm{nl}) ; \mathbf{n}=\mathbf{3}-\mathbf{5}$ nl Initial state of the $\mathrm{Na}$ atom

Section 8.3: Proton-impact target-excitation cross sections from $\mathrm{Na}\left(\mathbf{n l} \rightarrow \mathbf{n}^{\prime} \mathbf{l}^{\prime}\right) ; \mathbf{n}, \mathbf{n}^{\prime}=\mathbf{3}-5$ $n l \rightarrow n^{\prime} l^{\prime}$ Initial - final states of the $\mathrm{Na}$ atom

Section 8.4: Proton-impact target-electron-loss cross sections from $\mathrm{Na}(\mathrm{nl}) ; \mathbf{n}=3-5$ nl Initial state of the $\mathrm{Na}$ atom 


\section{Tables}

\begin{tabular}{|c|c|c|c|c|c|c|c|c|}
\hline $\mathbf{n l} \rightarrow \mathbf{n}^{\prime} \mathbf{l}^{\prime}$ & $\Delta \mathbf{E}$ & $\mathbf{A}_{1}$ & $\mathbf{A}_{2}$ & $\mathbf{A}_{3}$ & $\mathbf{A}_{4}$ & $\mathbf{A}_{5}$ & $\mathbf{A}_{6}$ & $\mathbf{A}_{7}$ \\
\hline $3 s \rightarrow 3 p$ & 2.09937 & -23.3231 & 38.358 & -5.70599 & -3.4816 & 27.1453 & 0.453874 & 14.7003 \\
\hline $3 \mathrm{~s} \rightarrow 3 \mathrm{~d}$ & 3.61642 & 54.0317 & -27.3057 & -28.6423 & 35.1405 & -0.288761 & 0.381653 & 1.04435 \\
\hline $3 \mathrm{~s} \rightarrow 4 \mathrm{~s}$ & 3.19192 & 2.51105 & -10.8455 & 21.3071 & -13.2455 & 0 & 0.58 & 11.3285 \\
\hline $3 \mathrm{~s} \rightarrow 4 \mathrm{p}$ & 3.75248 & 10.5845 & -44.94 & 74.7574 & -40.3895 & 2.12788 & $5.9750 \cdot 10^{-3}$ & 1.67677 \\
\hline $3 \mathrm{~s} \rightarrow 4 \mathrm{~d}$ & 4.28447 & 6.22968 & 10.1143 & -34.6957 & 32.5314 & 0.13928 & 1.08379 & 2.04554 \\
\hline $3 \mathrm{~s} \rightarrow 4 \mathrm{f}$ & 4.28855 & 0.753717 & 13.9336 & -35.7825 & 31.9556 & 0.0297361 & 0.814152 & 1.67582 \\
\hline $3 \mathrm{~s} \rightarrow 5 \mathrm{~s}$ & 4.11711 & 18.3199 & -52.2671 & 52.2258 & 4.31253 & 0.877115 & 0.504837 & 0.253928 \\
\hline $3 \mathrm{p} \rightarrow 3 \mathrm{~d}$ & 1.51705 & -274.892 & 380.104 & -38.007 & -51.4476 & 208.52 & $3.6757 \cdot 10^{-3}$ & 2.21269 \\
\hline $3 p \rightarrow 4 s$ & 1.09255 & -8.15967 & 19.8158 & -29.2698 & 28.5195 & 5.47456 & 0.948679 & 24.7919 \\
\hline $3 p \rightarrow 4 p$ & 1.65311 & 4.87684 & -11.1746 & 16.1142 & -6.00012 & 0.100725 & 0.791831 & 14.9378 \\
\hline $3 \mathrm{p} \rightarrow 4 \mathrm{~d}$ & 2.1851 & -2.1686 & -36.4101 & 86.9806 & -38.8431 & 16.307 & 0.06977 & 2.2177 \\
\hline $3 p \rightarrow 4 f$ & 2.18918 & 2.3848 & 3.1897 & -19.6921 & 21.5142 & -0.0105359 & 0.839963 & 15.4706 \\
\hline $3 p \rightarrow 5 s$ & 2.01774 & 1.2911 & -14.0441 & 29.5538 & -16.5211 & 1.56757 & 0.02045 & 3.2114 \\
\hline $3 \mathrm{~d} \rightarrow 4 \mathrm{p}$ & 0.13606 & -4.17001 & -10.1151 & 137.921 & -59.7171 & 11.0426 & 3.96822 & 60.5028 \\
\hline $3 \mathrm{~d} \rightarrow 4 \mathrm{~d}$ & 0.668046 & 4.64022 & 15.006 & -147.95 & 270.543 & 0.176582 & 2.6348 & 46.0857 \\
\hline $3 \mathrm{~d} \rightarrow 4 \mathrm{f}$ & 0.672127 & $-1.6638 \cdot 10^{3}$ & $1.5201 \cdot 10^{3}$ & 334.707 & $4.8787 \cdot 10^{3}$ & $1.0803 \cdot 10^{3}$ & 2.38353 & 1.18888 \\
\hline $3 \mathrm{~d} \rightarrow 5 \mathrm{~s}$ & 0.50069 & 0.354729 & 11.0975 & -38.9064 & 67.6696 & 0.0158897 & 2.77575 & 50.1735 \\
\hline $4 \mathrm{~s} \rightarrow 3 \mathrm{~d}$ & 0.4245 & 9.83953 & -61.7689 & 180.035 & -89.1436 & 0.208417 & 2.5022 & 49.9816 \\
\hline $4 \mathrm{~s} \rightarrow 4 \mathrm{p}$ & 0.56056 & -766.196 & $1.45 \cdot 10^{3}$ & 0 & 0 & 539.17 & 3.36726 & 4.00164 \\
\hline $4 \mathrm{~s} \rightarrow 4 \mathrm{~d}$ & 1.09255 & -1.05183 & -73.0017 & 371.593 & -654.485 & -0.128653 & 4.49224 & -47.2604 \\
\hline $4 \mathrm{~s} \rightarrow 4 \mathrm{f}$ & 1.09663 & -3.00114 & -58.8159 & 333.103 & -675.733 & -0.11054 & 4.49224 & -36.4141 \\
\hline $4 \mathrm{~s} \rightarrow 5 \mathrm{~s}$ & 0.92519 & 7.40763 & -52.1312 & 116.889 & -54.6344 & 1.06354 & 1.63344 & 10.6933 \\
\hline $4 \mathrm{p} \rightarrow 4 \mathrm{~d}$ & 0.531986 & $-2.1967 \cdot 10^{3}$ & $3.4305 \cdot 10^{5}$ & $-1.8810 \cdot 10^{6}$ & $6.1742 \cdot 10^{5}$ & $-2.2207 \cdot 10^{4}$ & 9.11098 & -0.0493211 \\
\hline $4 \mathrm{p} \rightarrow 4 \mathrm{f}$ & 0.536067 & 411.469 & $-2.3604 \cdot 10^{3}$ & $4.932 \cdot 10^{3}$ & $-2.3127 \cdot 10^{3}$ & 14.533 & 1.80954 & 1.5026 \\
\hline $4 \mathrm{p} \rightarrow 5 \mathrm{~s}$ & 0.36463 & -6.70865 & -211.981 & $1.3883 \cdot 10^{3}$ & -522.46 & 16.0299 & 8.01689 & 36.2413 \\
\hline $4 \mathrm{~d} \rightarrow 4 \mathrm{f}^{\dagger}$ & $4.08 \cdot 10^{-3}$ & -335.85 & $-1.7174 \cdot 10^{3}$ & 411.336 & -2.99991 & 330.533 & $3.3763 \cdot 10^{-5}$ & 11.1878 \\
\hline $5 \mathrm{~s} \rightarrow 4 \mathrm{~d}$ & 0.167356 & 4.25353 & -45.5338 & 562.593 & -151.995 & $3.0812 \cdot 10^{-3}$ & 16.3001 & 560.193 \\
\hline $5 \mathrm{~s} \rightarrow 4 \mathrm{f}$ & 0.171437 & 12.4203 & -126.653 & 615.687 & -306.243 & -0.041334 & 4.36124 & 34.695 \\
\hline
\end{tabular}

Table 1

Fit Parameters for Electron-Impact Target Excitation Cross Section of $\mathrm{Na}\left(n l \rightarrow n^{\prime} l^{\prime}\right) ; n, n^{\prime}=3-5$

See page 16 for Explanation of Tables

$\dagger$ The fit parameters for $4 \mathrm{~d} \rightarrow 4 \mathrm{f}$ are only valid above $0.2 \mathrm{eV}$.

Tables - 1 


\begin{tabular}{|c||c|c||c|c|c|c|c|c|c|}
\hline $\mathbf{n l}$ & $\mathbf{I}_{\mathbf{n l}}$ & $\mathbf{E}_{\text {low }}$ & $\mathbf{A}_{\mathbf{1}}$ & $\mathbf{A}_{\mathbf{2}}$ & $\mathbf{A}_{\mathbf{3}}$ & $\mathbf{A}_{\mathbf{4}}$ & $\mathbf{A}_{\mathbf{5}}$ & $\mathbf{A}_{\mathbf{6}}$ & $\mathbf{A}_{\mathbf{7}}$ \\
\hline $3 \mathrm{~s}$ & 5.13891 & 5.2 & 0 & 66.4483 & 24.5645 & 101.03 & -5.0082 & 0 & $4.37 \cdot 10^{-3}$ \\
$3 \mathrm{p}$ & 3.03954 & 3.2 & 20.8332 & -13.1449 & -19.4089 & 33.258 & 0.542801 & -56.3793 & 0.0379105 \\
$3 \mathrm{~d}$ & 1.52249 & 2.08 & -105.202 & -21.5898 & 485.919 & -337.711 & 0.369663 & 331.13 & 0.0351848 \\
$4 \mathrm{~s}$ & 1.94699 & 2.5 & -0.729955 & -0.0916904 & 3.32036 & -2.34172 & $9.0515 \cdot 10^{-5}$ & 2.25425 & 3.72267 \\
$4 \mathrm{p}$ & 1.38643 & 1.9 & -28.9039 & -6.49469 & 130.161 & -92.2872 & 0.19249 & 91.127 & 0.176319 \\
$4 \mathrm{~d}$ & 0.850363 & 1.7 & 0.0711827 & 7.87424 & -17.7004 & 8.79277 & -0.0200515 & -5.48488 & -0.446075 \\
$4 \mathrm{f}$ & 0.850363 & 1.3 & 1.8088 & 2.13387 & -12.4468 & 8.35135 & $-6.259 \cdot 10^{-3}$ & -6.66904 & -2.2193 \\
$5 \mathrm{~s}$ & 1.0218 & 1.5 & -3.83331 & -2.65103 & 21.742 & -14.8678 & $2.5528 \cdot 10^{-3}$ & 13.1159 & 1.28082 \\
\hline
\end{tabular}

Table 2

Fit Parameters for Electron-Impact Target Ionization Cross Sections of the valence electron in $\mathrm{Na}(\mathrm{nl}) ; n=3-5$ See page 16 for Explanation of Tables.

Fits containing inner-shell ionization can be obtained using Eq.(4) \& (3) together with Tab. D.

Tables - 2 


\begin{tabular}{|c||c||c|c|c|c|c|c|}
\hline $\mathbf{n l} \rightarrow \mathbf{n}^{\prime} \mathbf{l}^{\prime}$ & $\mathbf{E}_{\mathbf{l o w}}$ & $\mathbf{A}_{\mathbf{1}}$ & $\mathbf{A}_{\mathbf{2}}$ & $\mathbf{A}_{\mathbf{3}}$ & $\mathbf{A}_{\mathbf{4}}$ & $\mathbf{A}_{\mathbf{5}}$ & $\mathbf{A}_{\mathbf{6}}$ \\
\hline $3 \mathrm{~s} \rightarrow 3 \mathrm{p}$ & 0.2 & 899.91 & 0.813273 & 0.172984 & 0.171027 & 1.33781 & -1.97329 \\
$3 \mathrm{~s} \rightarrow 3 \mathrm{~d}$ & 0.2 & 2.35787 & 16.0799 & $1.661 \cdot 10^{8}$ & 1.75626 & 1.88912 & -3.32454 \\
$3 \mathrm{~s} \rightarrow 4 \mathrm{~s}$ & 0.2 & 0.5291 & 11.1454 & 13.8 & 2.21 & 0.9258 & -2.8909 \\
$3 \mathrm{~s} \rightarrow 4 \mathrm{p}$ & 0.3 & 12.287 & 1.00965 & 0.19894 & -3.3202 & 4.7024 & -6.149 \\
$3 \mathrm{~s} \rightarrow 4 \mathrm{~d}$ & 1 & 0.09605 & 35.1151 & 0 & 6.5924 & 2.4005 & -5.09985 \\
$3 \mathrm{~s} \rightarrow 4 \mathrm{f}$ & 1 & 0.4652 & 44.0372 & 0 & 1.51204 & 2.38218 & -5.305 \\
$3 \mathrm{~s} \rightarrow 5 \mathrm{~s}$ & 0.6 & 21.912 & 67.9026 & 2.72934 & $3.5101 \cdot 10^{-3}$ & 0.941943 & -3.9001 \\
$3 \mathrm{p} \rightarrow 3 \mathrm{~d}$ & 0.3 & 445.154 & 9.8646 & 0 & 0.0851 & 0.8208 & -2.1403 \\
$3 \mathrm{p} \rightarrow 4 \mathrm{~s}$ & 0.2 & 875.942 & 0.0845244 & 3.2671 & 1.4616 & 0.12061 & 1.42592 \\
$3 \mathrm{p} \rightarrow 4 \mathrm{p}$ & 0.3 & 13.2437 & 4.764 & 2.91617 & 1.44602 & 2.30177 & -1.72801 \\
$3 \mathrm{p} \rightarrow 4 \mathrm{~d}$ & 0.2 & 795.051 & 4.7911 & 3.2671 & $1.7546 \cdot 10^{-3}$ & 0.265715 & -1.10414 \\
$3 \mathrm{p} \rightarrow 4 \mathrm{f}$ & 0.5 & 17.6253 & 9.36216 & 0 & 0.892868 & $7.8587 \cdot 10^{-3}$ & 0.782358 \\
$3 \mathrm{p} \rightarrow 5 \mathrm{~s}$ & 0.5 & 5.7944 & 6.98495 & 1.13626 & 107.387 & 6.9904 & -7.1283 \\
$3 \mathrm{~d} \rightarrow 4 \mathrm{p}$ & 1 & 301.658 & 30.0346 & 0 & 35.9391 & 4.67224 & -0.758748 \\
$3 \mathrm{~d} \rightarrow 4 \mathrm{~d}$ & 0.2 & 30.3405 & 6.373 & 0 & 0.2148 & 0.2718 & -1.382 \\
$3 \mathrm{~d} \rightarrow 4 \mathrm{f}$ & 0.2 & 8.8668 & 12.0228 & 1.26008 & -1.73829 & 0.0960184 & -1.04151 \\
$3 \mathrm{~d} \rightarrow 5 \mathrm{~s}$ & 0.3 & 1.97042 & 2.82693 & 0 & 36.1299 & 8.32895 & -0.842273 \\
$4 \mathrm{~s} \rightarrow 3 \mathrm{~d}$ & 0.2 & 76.102 & 0.108 & $1.4 \cdot 10^{3}$ & 4.79189 & 27.7 & -45.7225 \\
$4 \mathrm{~s} \rightarrow 4 \mathrm{p}$ & 0.7 & $3.0 \cdot 10^{3}$ & 0.181647 & 0.0152269 & -1.00172 & 0.264541 & 0.553659 \\
$4 \mathrm{~s} \rightarrow 4 \mathrm{~d}$ & 0.3 & 12.2821 & 7.48675 & 0 & 41.5655 & 2.9866 & -2.8764 \\
$4 \mathrm{~s} \rightarrow 4 \mathrm{f}$ & 0.2 & 18.126 & 7.2308 & 0 & 0.3843 & 1.20817 & -4.77267 \\
$4 \mathrm{~s} \rightarrow 5 \mathrm{~s}$ & 1 & 4.57798 & 73.0856 & 11.2459 & 55.6678 & 3.64108 & 1.22198 \\
$4 \mathrm{p} \rightarrow 4 \mathrm{~d}$ & 0.2 & $1.5105 \cdot 10^{3}$ & 41.0849 & 1.13114 & 5.48059 & 6.99795 & -2.70534 \\
$4 \mathrm{p} \rightarrow 4 \mathrm{f}$ & 0.2 & 98.1211 & 5.2274 & 0 & 0.1986 & 0.9862 & -3.3092 \\
$4 \mathrm{p} \rightarrow 5 \mathrm{~s}$ & 0.2 & 695.167 & 0.379397 & $1.2862 \cdot 10^{-3}$ & -0.0266469 & 4.46338 & -12.1889 \\
$4 \mathrm{~d} \rightarrow 4 \mathrm{f}$ & 0.4 & $2.0 \cdot 10^{3}$ & 28.7819 & 0 & 35.9994 & 2.90774 & -0.0355274 \\
$5 \mathrm{~s} \rightarrow 4 \mathrm{~d}$ & 0.4 & 352.156 & 10.148 & 0 & 177.117 & 6.12077 & -3.60091 \\
$5 \mathrm{~s} \rightarrow 4 \mathrm{f}$ & 0.4 & 50.441 & 12.0566 & 0 & -2.32538 & 0.333314 & -0.669005 \\
\hline
\end{tabular}

Table 3

Fit parameters for proton-impact target excitation cross sections of $\mathrm{Na}\left(n l \rightarrow n^{\prime} l^{\prime}\right) ; n, n^{\prime}=3-5$ See page 16 for Explanation of Tables

Tables - 3 


\begin{tabular}{|c||c|c|c|c|c|c|}
\hline $\mathbf{n l} \rightarrow \mathbf{n}^{\prime} \mathbf{l}^{\prime}$ & $\mathbf{A}_{\mathbf{7}}$ & $\mathbf{A}_{\mathbf{8}}$ & $\mathbf{A}_{\mathbf{9}}$ & $\mathbf{A}_{\mathbf{1 0}}$ & $\mathbf{A}_{\mathbf{1 1}}$ & $\mathbf{A}_{\mathbf{1 2}}$ \\
\hline 3s $\rightarrow 3 \mathrm{p}$ & -9.90738 & 1.82931 & 20.1605 & 0.899862 & 0.934209 & -0.012689 \\
$3 \mathrm{~s} \rightarrow 3 \mathrm{~d}$ & 9.56875 & 14.1112 & 0.0318923 & 1.56873 & 1 & 9.13887 \\
3s $\rightarrow 4 \mathrm{~s}$ & -6.0985 & 4.29572 & $2.6 \cdot 10^{-3}$ & 2.1235 & 1.4632 & 93.1684 \\
$3 \mathrm{~s} \rightarrow 4 \mathrm{p}$ & 69.9622 & 30.4996 & 3.1234 & 1.48366 & 1.38235 & -0.32704 \\
$3 \mathrm{~s} \rightarrow 4 \mathrm{~d}$ & 21.6803 & 7.40333 & $2.7 \cdot 10^{-3}$ & 1.91665 & 1 & 272.253 \\
3s $\rightarrow 4 \mathrm{f}$ & 178.707 & 27.0857 & 0.0628 & 2.30125 & $1.0 \cdot 10^{-10}$ & 29.7278 \\
$3 \mathrm{~s} \rightarrow 5 \mathrm{~s}$ & -43.6875 & 68.1887 & 1.82419 & 0.967314 & 6.44834 & 22.6012 \\
$3 \mathrm{p} \rightarrow 3 \mathrm{~d}$ & -6.61 & 7.0552 & 0.2111 & 1.0887 & 1 & 26.1162 \\
$3 \mathrm{p} \rightarrow 4 \mathrm{~s}$ & -95.267 & -0.041652 & 13.4883 & 0.92967 & 0.126126 & 4.83299 \\
$3 \mathrm{p} \rightarrow 4 \mathrm{p}$ & 34.7144 & 27.6903 & 0.19179 & 1.8542 & 0.525469 & 3.12512 \\
$3 \mathrm{p} \rightarrow 4 \mathrm{~d}$ & -238.849 & 4.67249 & 6.89654 & 0.97723 & -0.0730905 & 32.9442 \\
$3 \mathrm{p} \rightarrow 4 \mathrm{f}$ & -6.63413 & -0.0354364 & 7.58916 & 1.01267 & 1 & 4.62623 \\
$3 \mathrm{p} \rightarrow 5 \mathrm{~s}$ & 8.6305 & 114.073 & 0.42788 & 1.3713 & -1.70258 & 1.675 \\
$3 \mathrm{~d} \rightarrow 4 \mathrm{p}$ & 39.6619 & 1.22105 & 3.74937 & 0.881504 & $2.8484 \cdot 10^{-7}$ & 24.8598 \\
$3 \mathrm{~d} \rightarrow 4 \mathrm{~d}$ & 2.0156 & 0.4264 & 0.8904 & 2.6894 & 1 & 15.5586 \\
$3 \mathrm{~d} \rightarrow 4 \mathrm{f}$ & 7.83512 & 0.303191 & 0.0418302 & 0.788086 & -1.89013 & 525.229 \\
$3 \mathrm{~d} \rightarrow 5 \mathrm{~s}$ & 22.1788 & 0.981295 & 0.077633 & 1.8594 & 1 & 26.1051 \\
$4 \mathrm{~s} \rightarrow 3 \mathrm{~d}$ & 74.7606 & 1.8727 & 7.4108 & 1.094 & -41.05 & -4.97677 \\
$4 \mathrm{~s} \rightarrow 4 \mathrm{p}$ & 67.7319 & 30.4687 & 17.8543 & 0.984028 & 1.78496 & 0.348832 \\
$4 \mathrm{~s} \rightarrow 4 \mathrm{~d}$ & 47.503 & 67.1931 & -0.91548 & 1.1443 & 0 & 100.0 \\
$4 \mathrm{~s} \rightarrow 4 \mathrm{f}$ & 1.0506 & 0.2524 & $1.1 \cdot 10^{-3}$ & 2.35044 & 1 & 13.724 \\
$4 \mathrm{~s} \rightarrow 5 \mathrm{~s}$ & 0.955863 & 0.0178534 & $3.9414 \cdot 10^{-4}$ & 1.95208 & -0.55916 & 61.0529 \\
$4 \mathrm{p} \rightarrow 4 \mathrm{~d}$ & 0.0721157 & 1.14453 & $4.4491 \cdot 10^{-4}$ & 2.07563 & 3.00072 & 2.48347 \\
$4 \mathrm{p} \rightarrow 4 \mathrm{f}$ & 0.5538 & 0.09157 & 0.0539 & 1.0939 & 1 & 9.278 \\
$4 \mathrm{p} \rightarrow 5 \mathrm{~s}$ & 0.276855 & 2.18819 & 0.0374898 & 0.954608 & 1.03331 & $-1.3017 \cdot 10^{-3}$ \\
$4 \mathrm{~d} \rightarrow 4 \mathrm{f}$ & 39.7245 & 0.089685 & 1.96664 & 0.943349 & $1.1334 \cdot 10^{-8}$ & 23.3783 \\
$5 \mathrm{~s} \rightarrow 4 \mathrm{~d}$ & 283.157 & 4.75692 & 5.15505 & 2.46864 & $8.1046 \cdot 10^{-4}$ & 20.1594 \\
$5 \mathrm{~s} \rightarrow 4 \mathrm{f}$ & 3.51249 & -0.454977 & 0.0406219 & 1.83821 & 1.71289 & 14.6884 \\
\hline
\end{tabular}

Table 4

(cont.) Fit parameters for proton-impact target excitation cross sections of $\mathrm{Na}\left(n l \rightarrow n^{\prime} l^{\prime}\right) ; n, n^{\prime}=3-5$ See page 16 for Explanation of Tables

Tables - 4 


\begin{tabular}{|c||c||c|c|c|c|c|c|}
\hline $\mathbf{n l}$ & $\mathbf{E}_{\text {low }}$ & $\mathbf{A}_{\mathbf{1}}$ & $\mathbf{A}_{\mathbf{2}}$ & $\mathbf{A}_{\mathbf{3}}$ & $\mathbf{A}_{\mathbf{4}}$ & $\mathbf{A}_{\mathbf{5}}$ & $\mathbf{A}_{\mathbf{6}}$ \\
\hline $3 \mathrm{~s}$ & 0.2 & 11.319 & 2.51212 & $1.0 \cdot 10^{5}$ & 2.26648 & 0.172847 & -0.73075 \\
$3 \mathrm{p}$ & 0.2 & 27.8562 & 1.5103 & $5.72 \cdot 10^{4}$ & 1.86786 & 0.0564 & 0.1081 \\
$3 \mathrm{~d}$ & 0.2 & 81.6464 & 1.4629 & 275.929 & 6.35454 & 0.372612 & -0.0253238 \\
$4 \mathrm{~s}$ & 0.2 & 14.5812 & 1.0669 & $5.16 \cdot 10^{13}$ & 27.661 & 0.249027 & 0.159263 \\
$4 \mathrm{p}$ & 0.2 & 31.4476 & 1.3675 & $3.1062 \cdot 10^{9}$ & 17.0752 & 0.665415 & -0.369477 \\
$4 \mathrm{~d}$ & 0.2 & 66.0274 & 0.259161 & $4.8614 \cdot 10^{6}$ & 942.831 & 8.1801 & -12.8437 \\
$4 \mathrm{f}$ & 0.2 & 76.1757 & 0.21478 & 131.206 & 20.4186 & 0.892532 & -0.184057 \\
$5 \mathrm{~s}$ & 0.2 & 27.7031 & 0.135768 & $2.0126 \cdot 10^{12}$ & $1.260 \cdot 10^{3}$ & 4.14424 & -3.75084 \\
\hline
\end{tabular}

Table 5

Fit Parameters for Proton-Impact Target Electron Loss from Na(nl); $n=3-5$

See page 16 for Explanation of Tables

Tables - 5 


\section{Graphs}

8.1. Electron-Impact Target Excitation Cross Sections
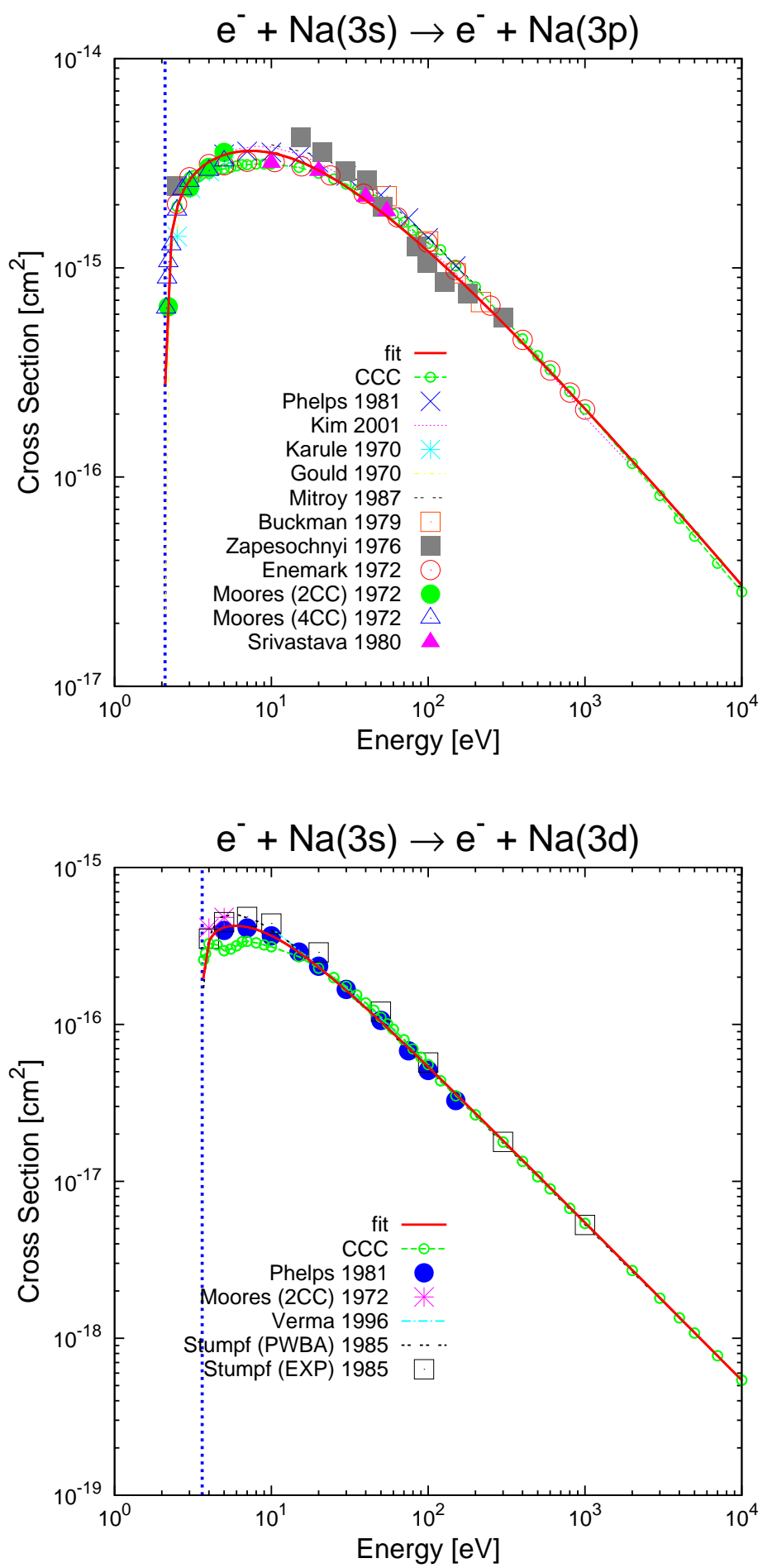

Graphs - 1 
Electron-impact target excitation (cont.)
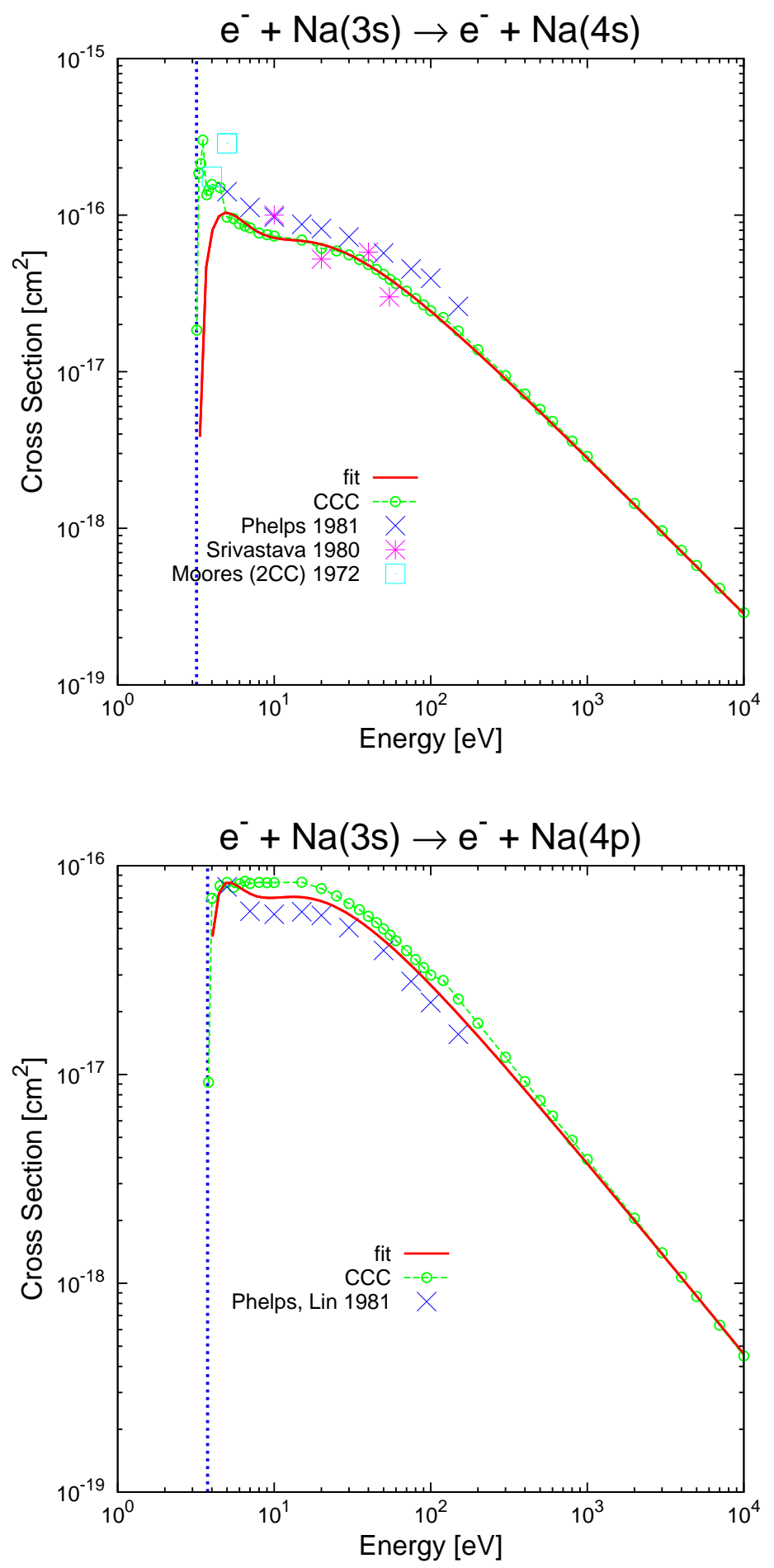

Graphs - 2 
Electron-impact target excitation (cont.)
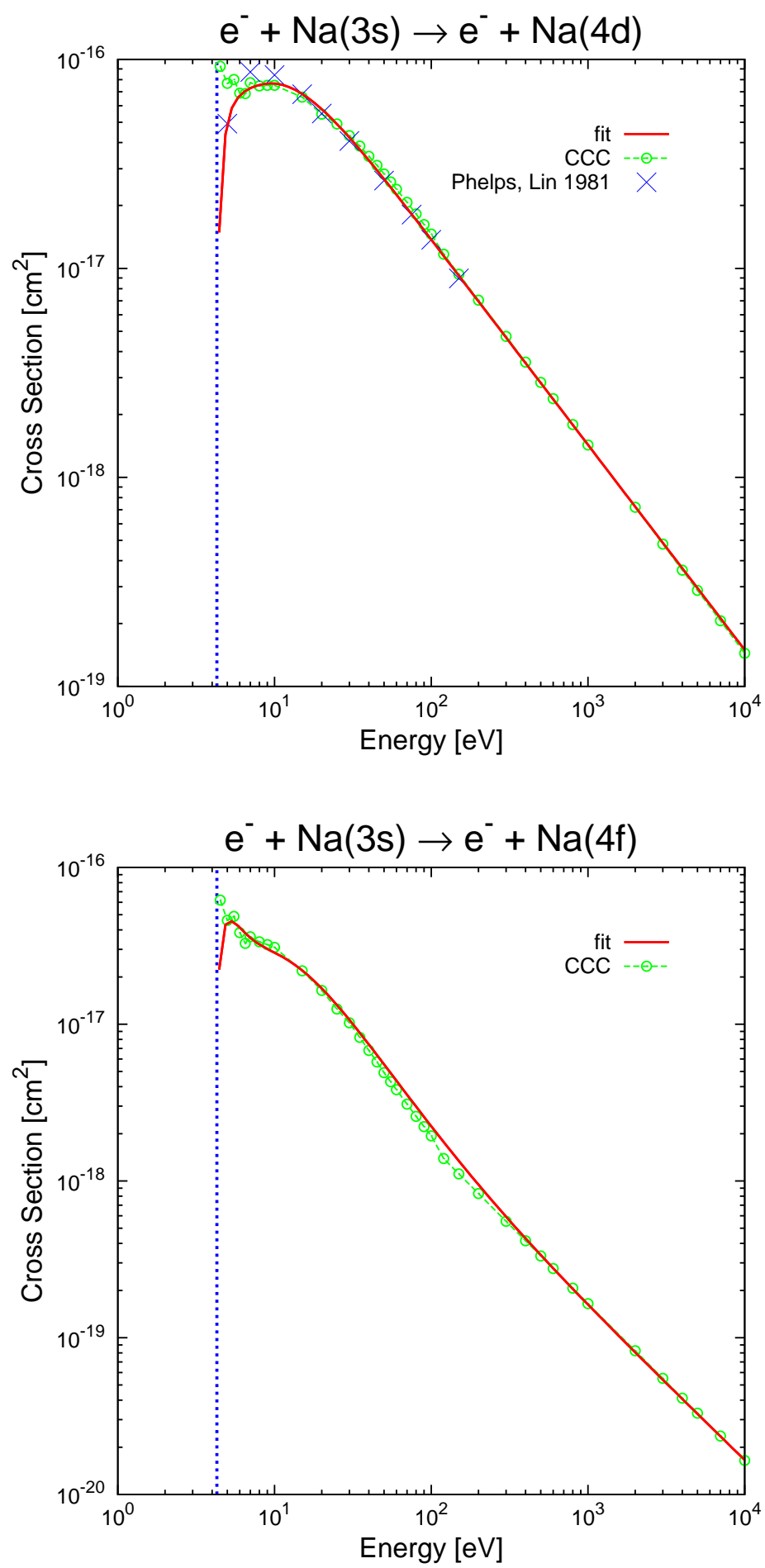

Graphs - 3 
Electron-impact target excitation (cont.)
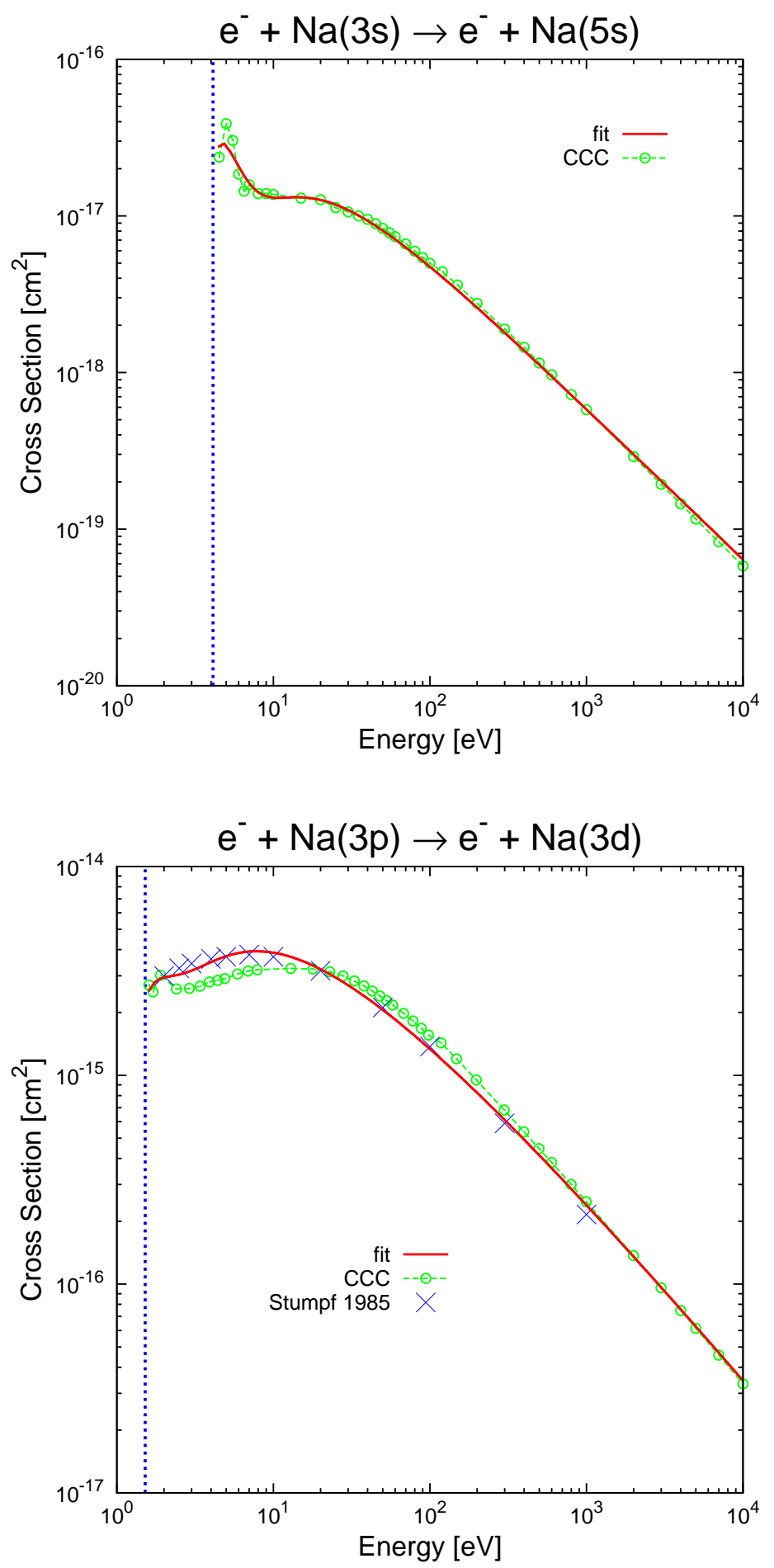

Graphs - 4 
Electron-impact target excitation (cont.)
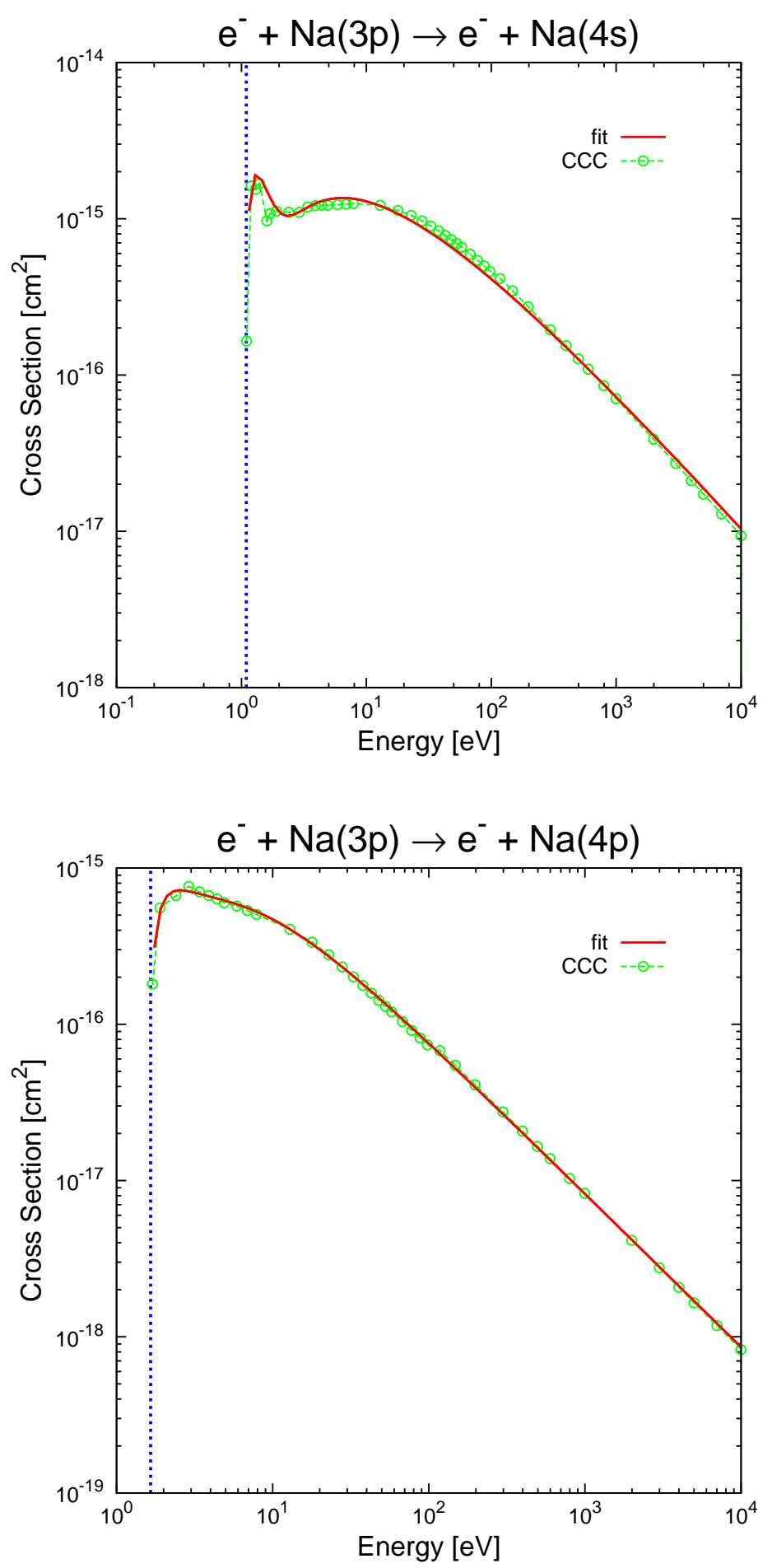

Graphs - 5 
Electron-impact target excitation (cont.)
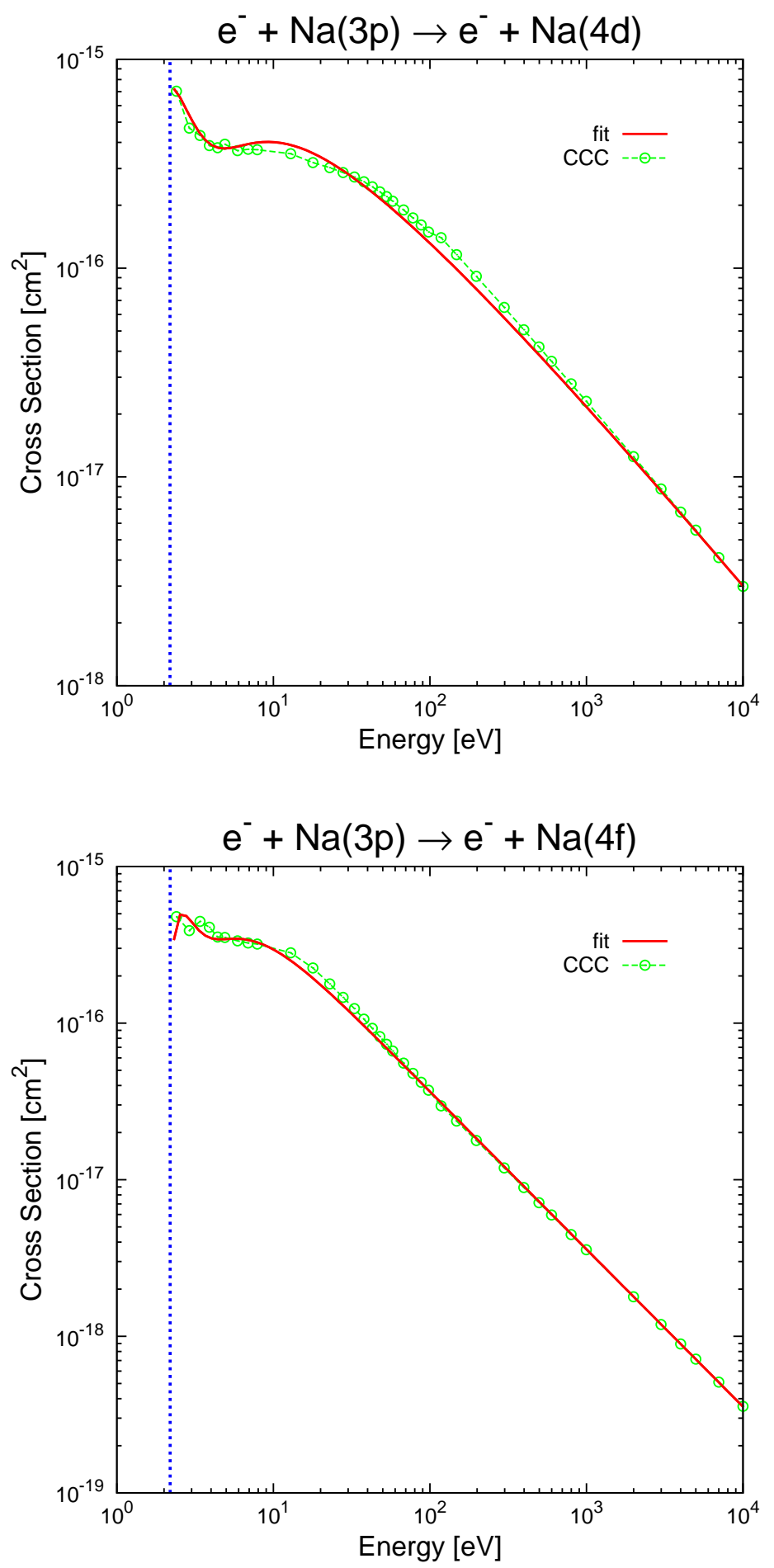

Graphs - 6 
Electron-impact target excitation (cont.)
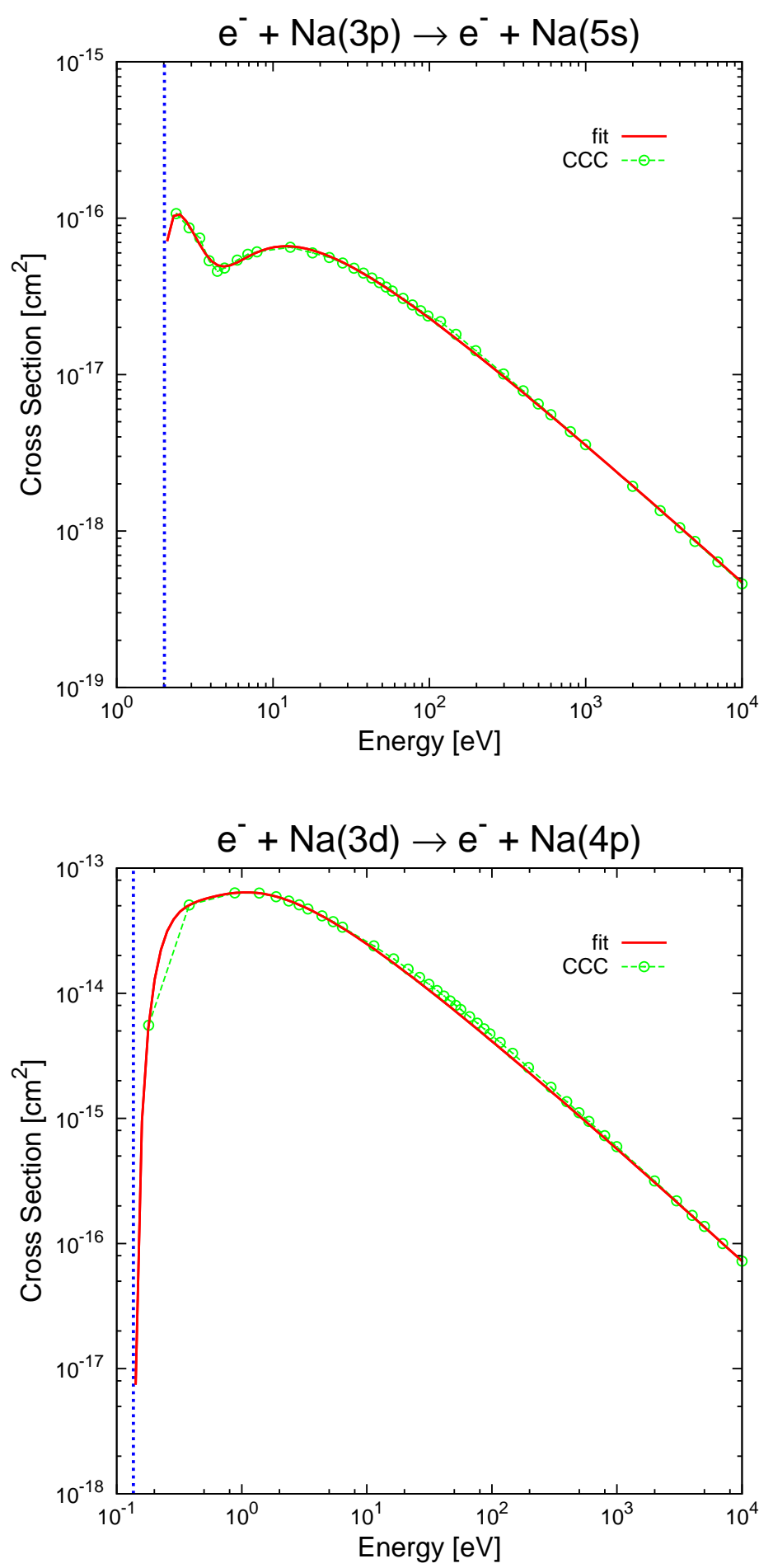

Graphs - 7 
Electron-impact target excitation (cont.)
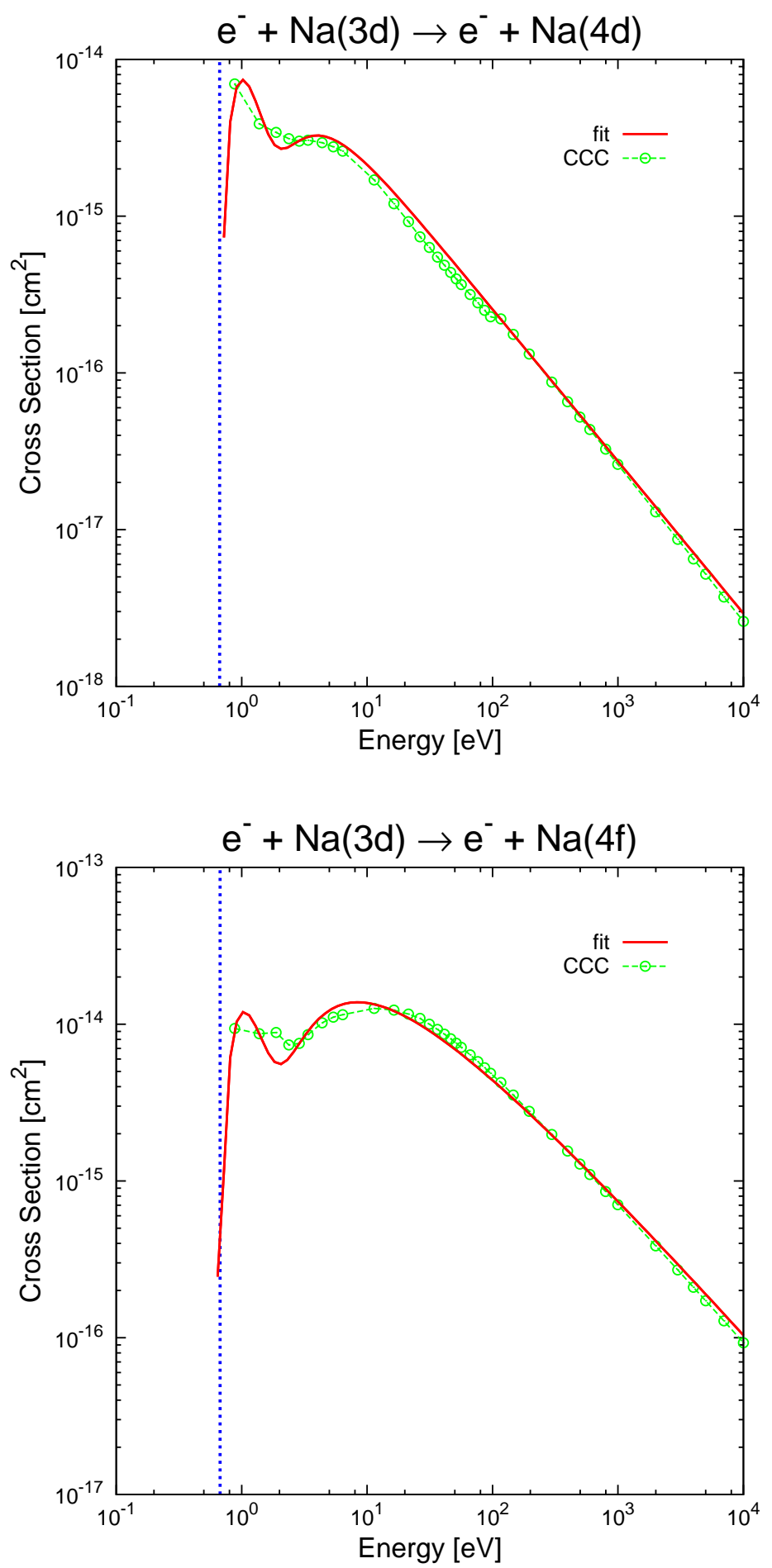

Graphs - 8 
Electron-impact target excitation (cont.)
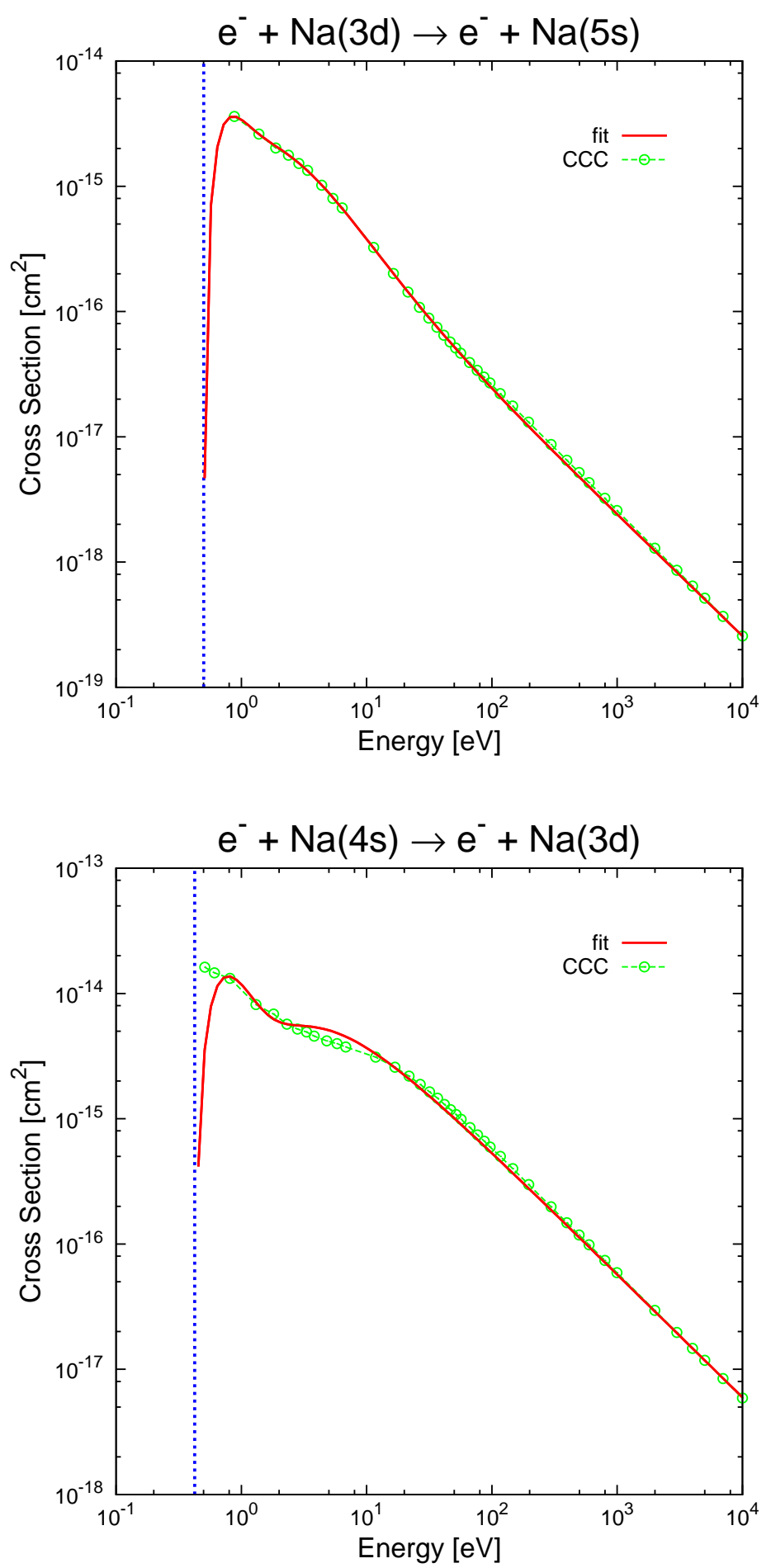

Graphs - 9 
Electron-impact target excitation (cont.)
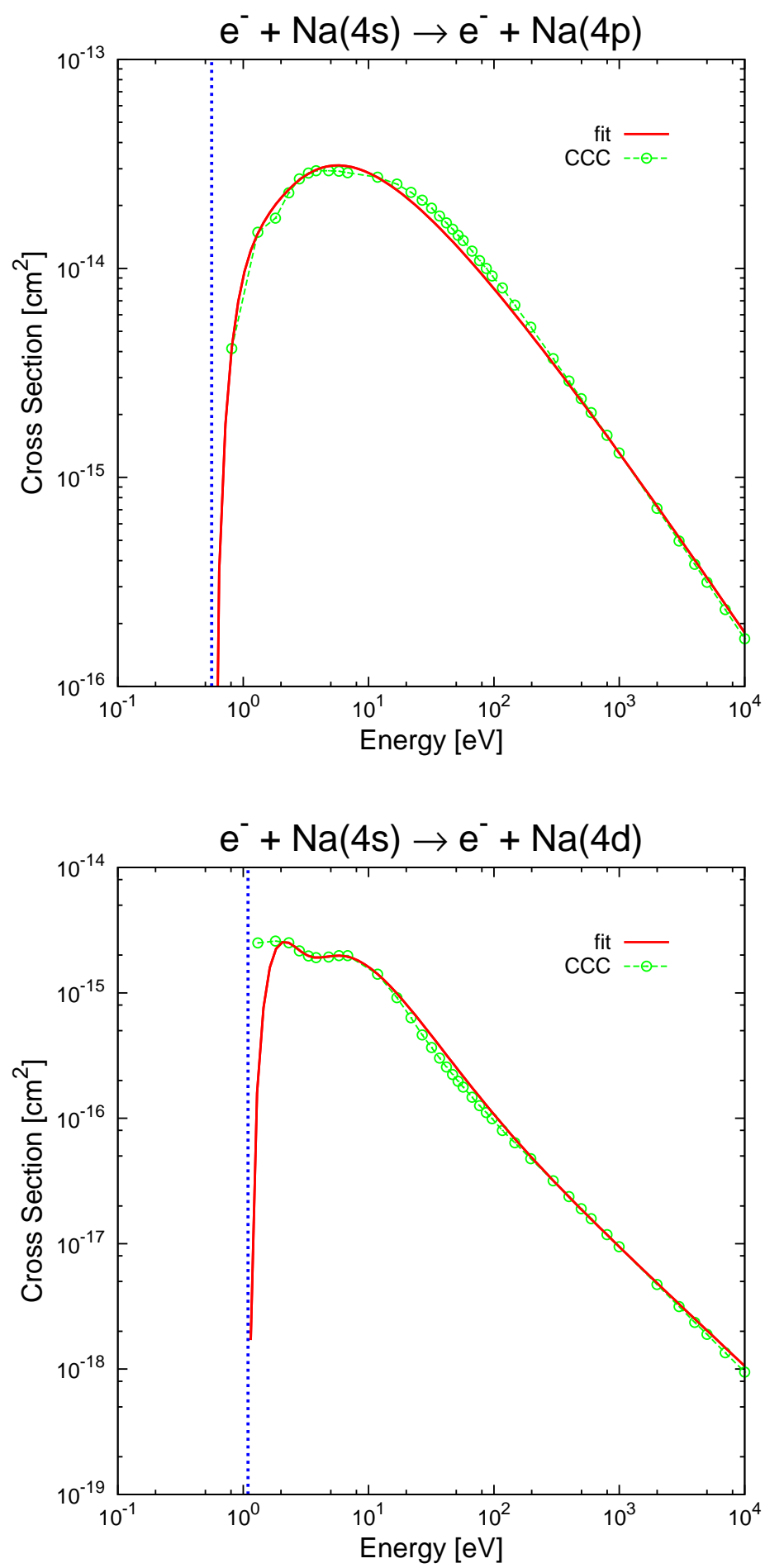

Graphs - 10 
Electron-impact target excitation (cont.)
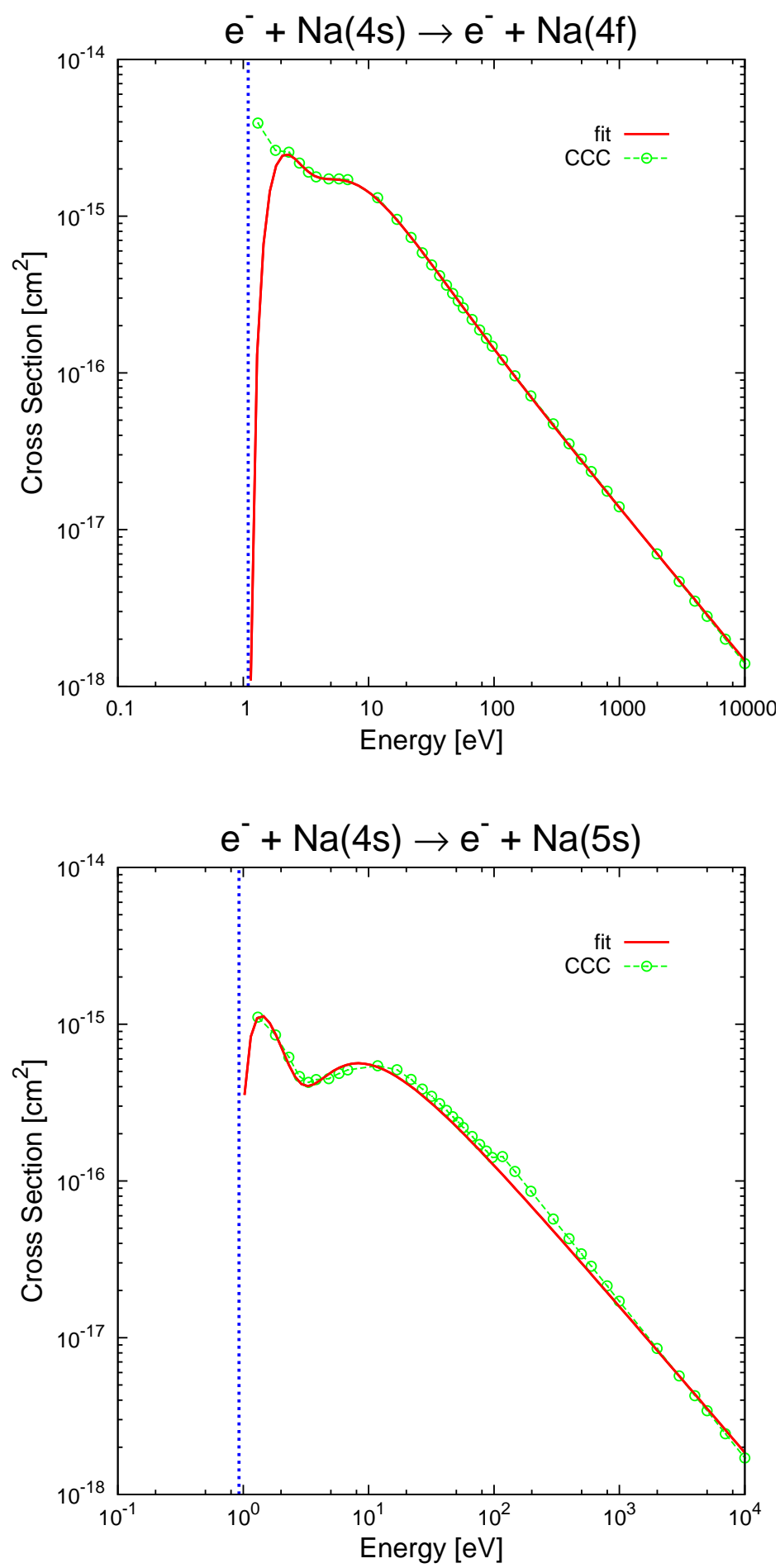

Graphs - 11 
Electron-impact target excitation (cont.)
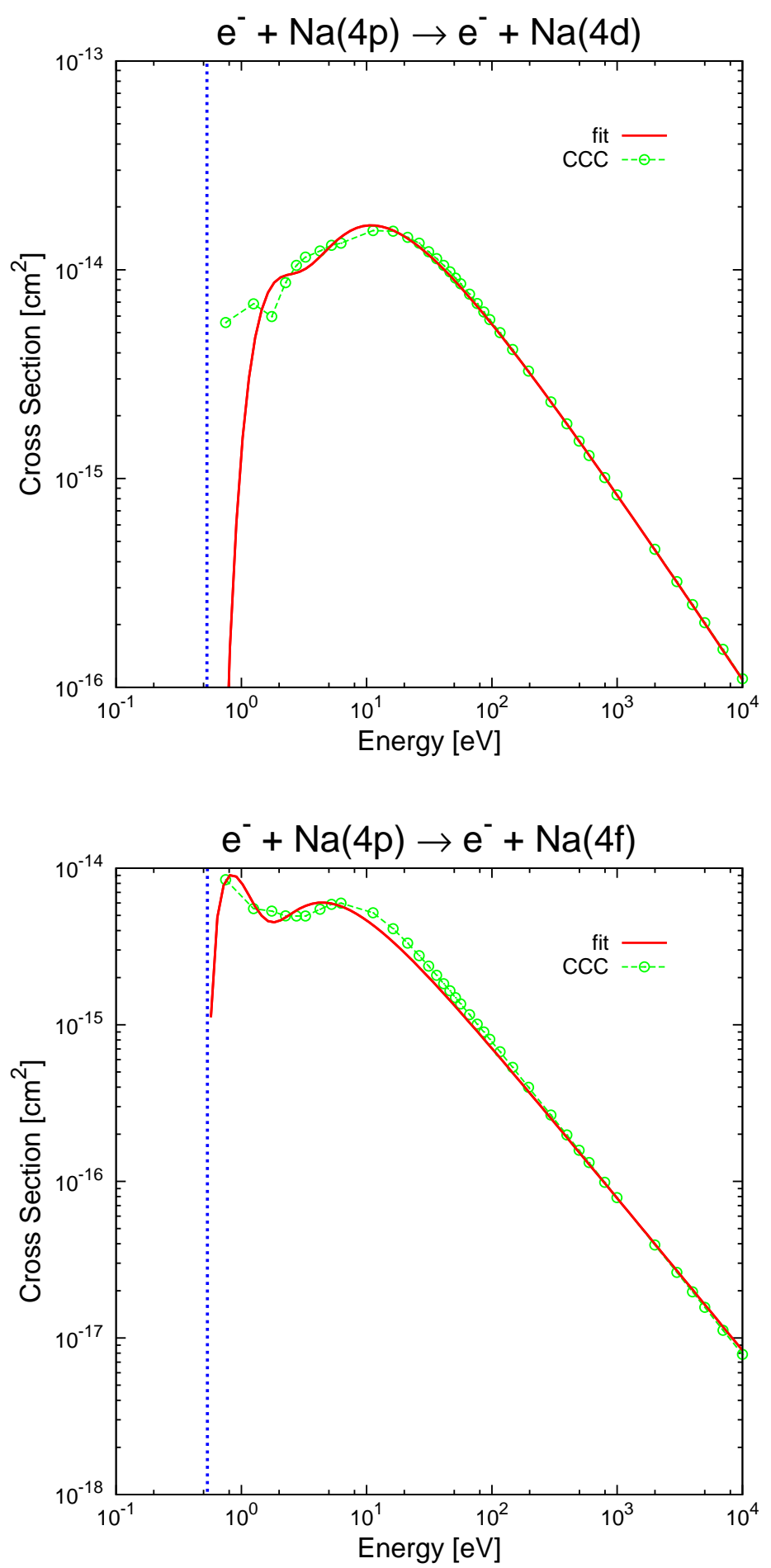

Graphs - 12 
Electron-impact target excitation (cont.)
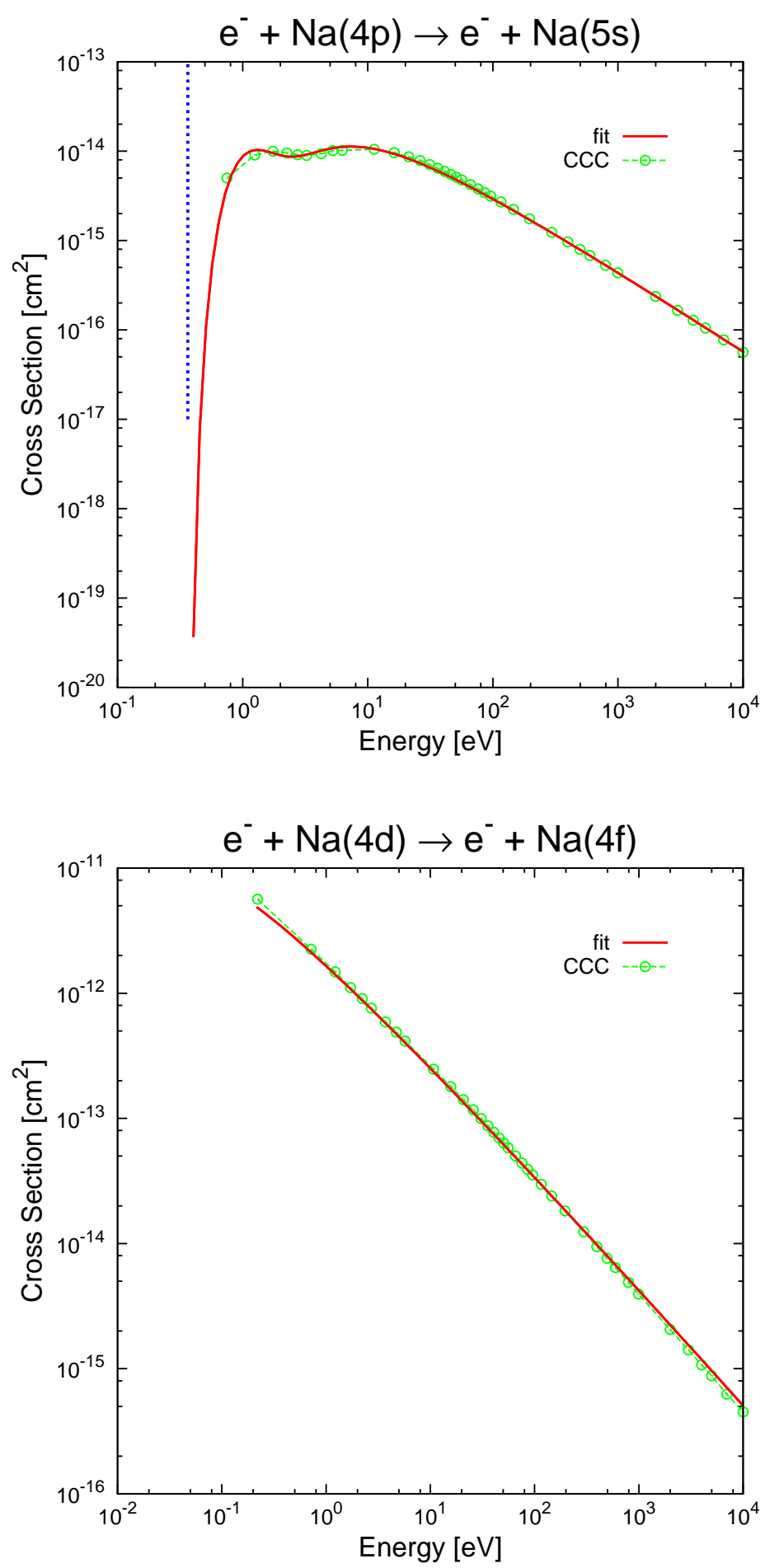

Graphs - 13 
Electron-impact target excitation (cont.)
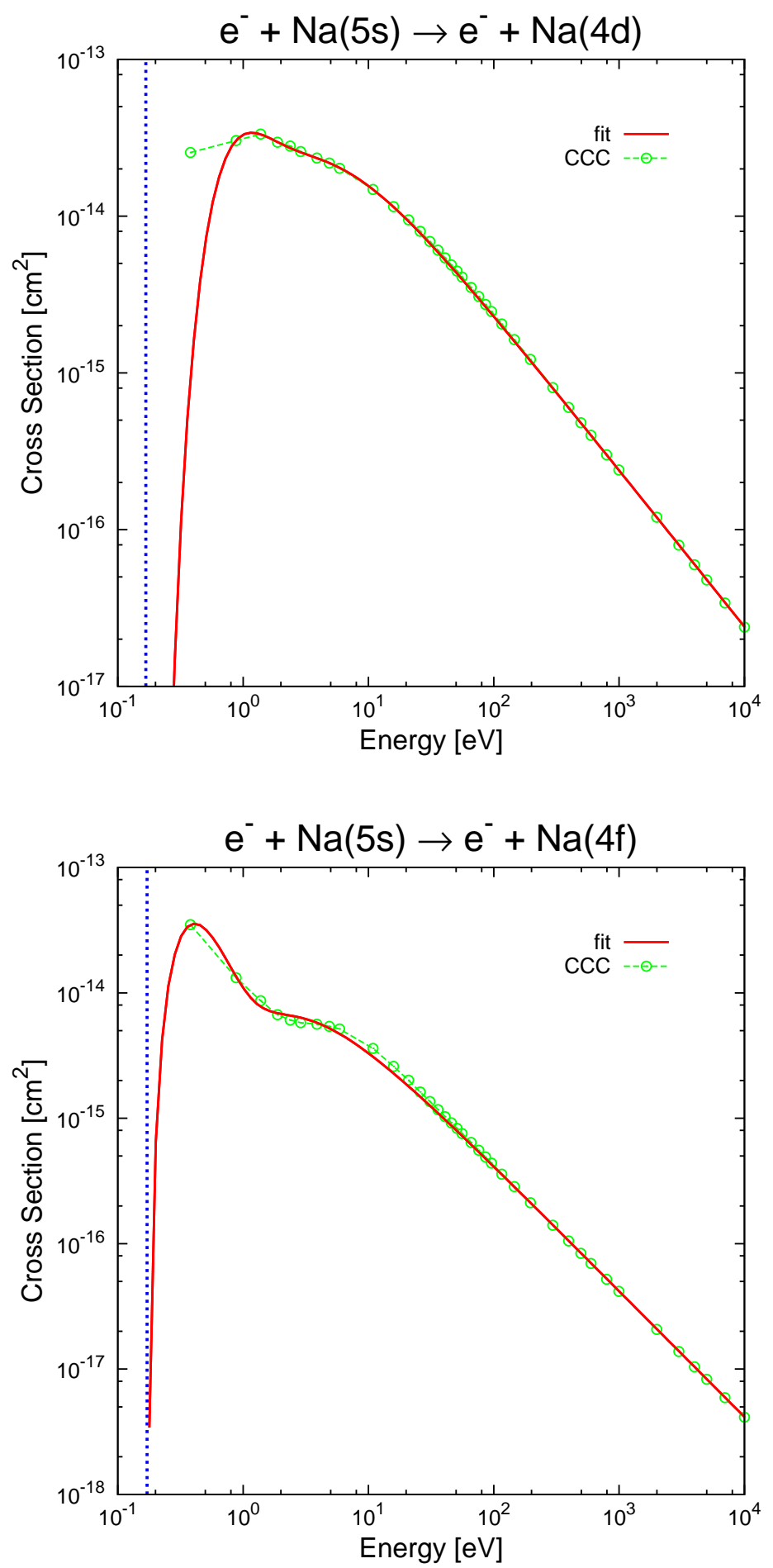

Graphs - 14 

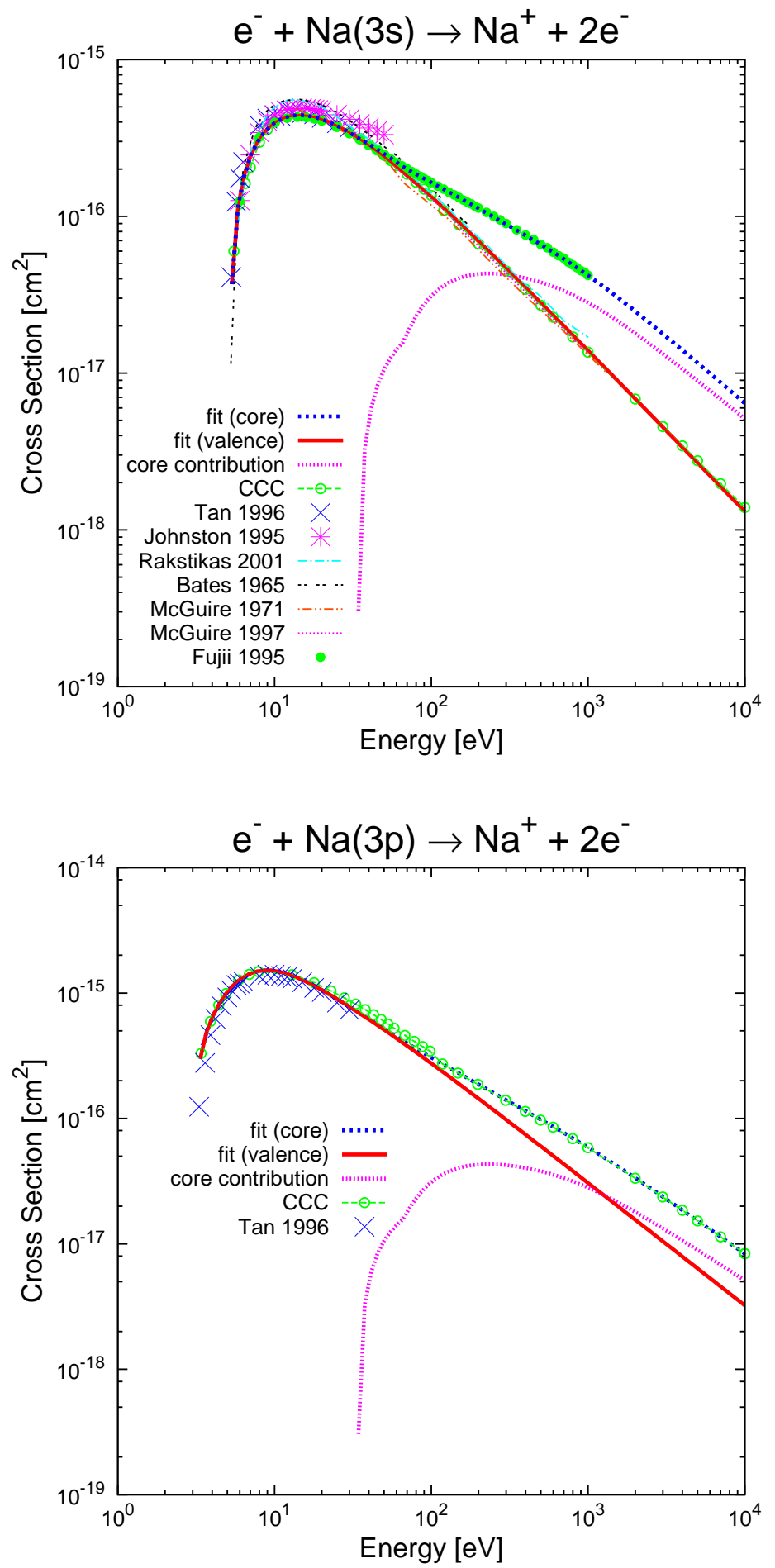

Graphs - 15 
Electron-impact target ionization (cont.)
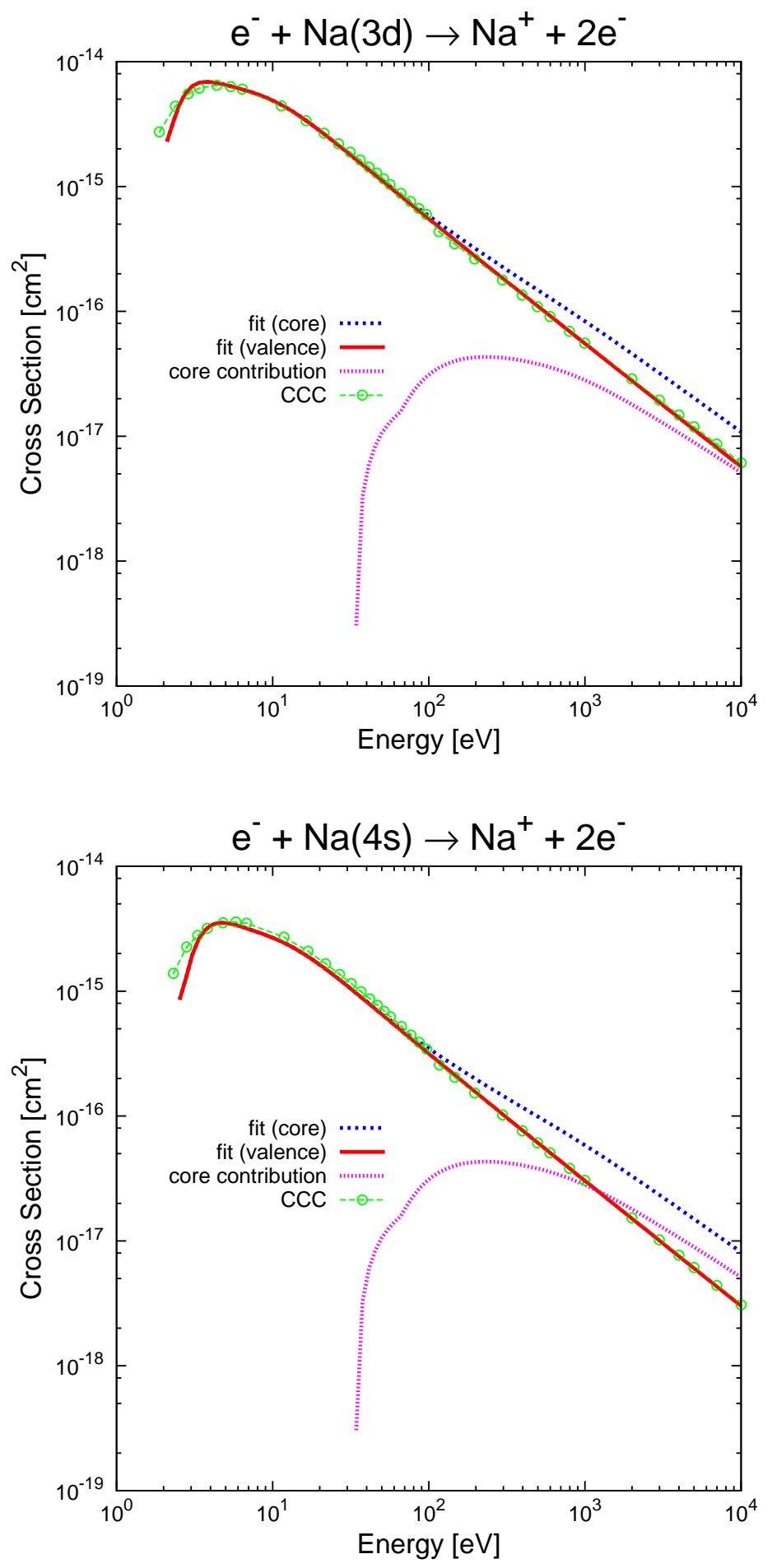

Graphs - 16 
Electron-impact target ionization (cont.)
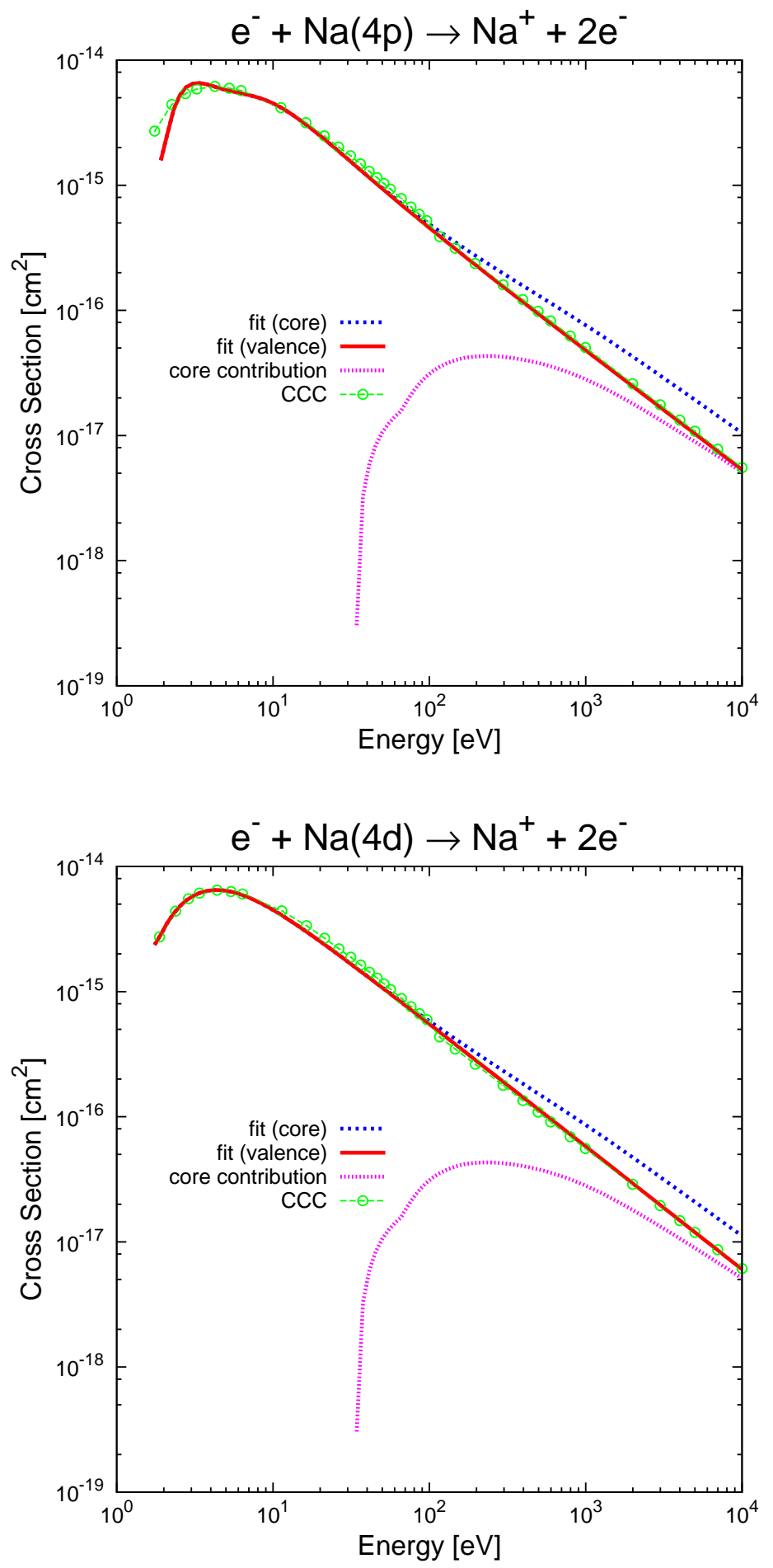

Graphs - 17 
Electron-impact target ionization (cont.)
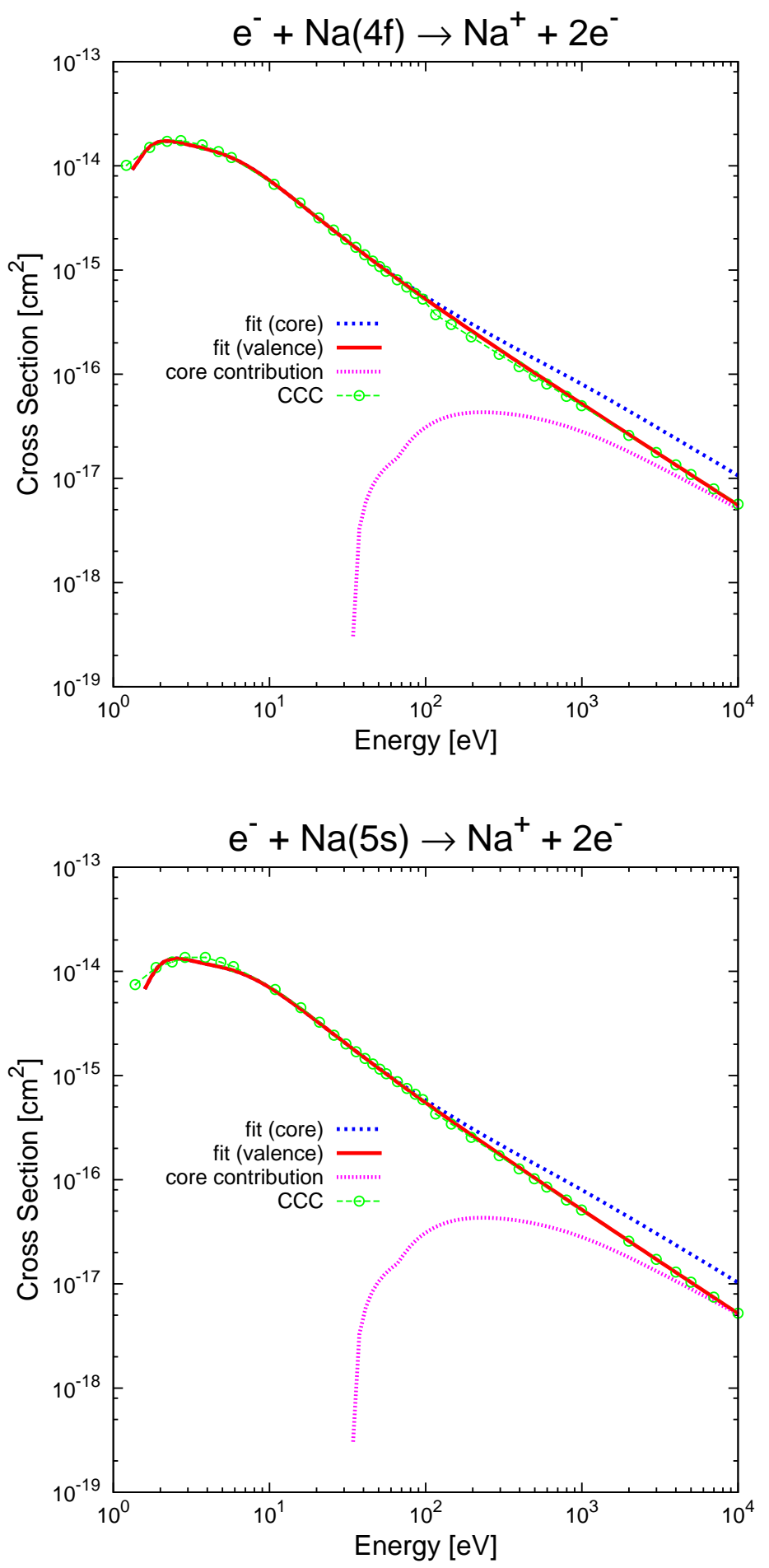

Graphs - 18 

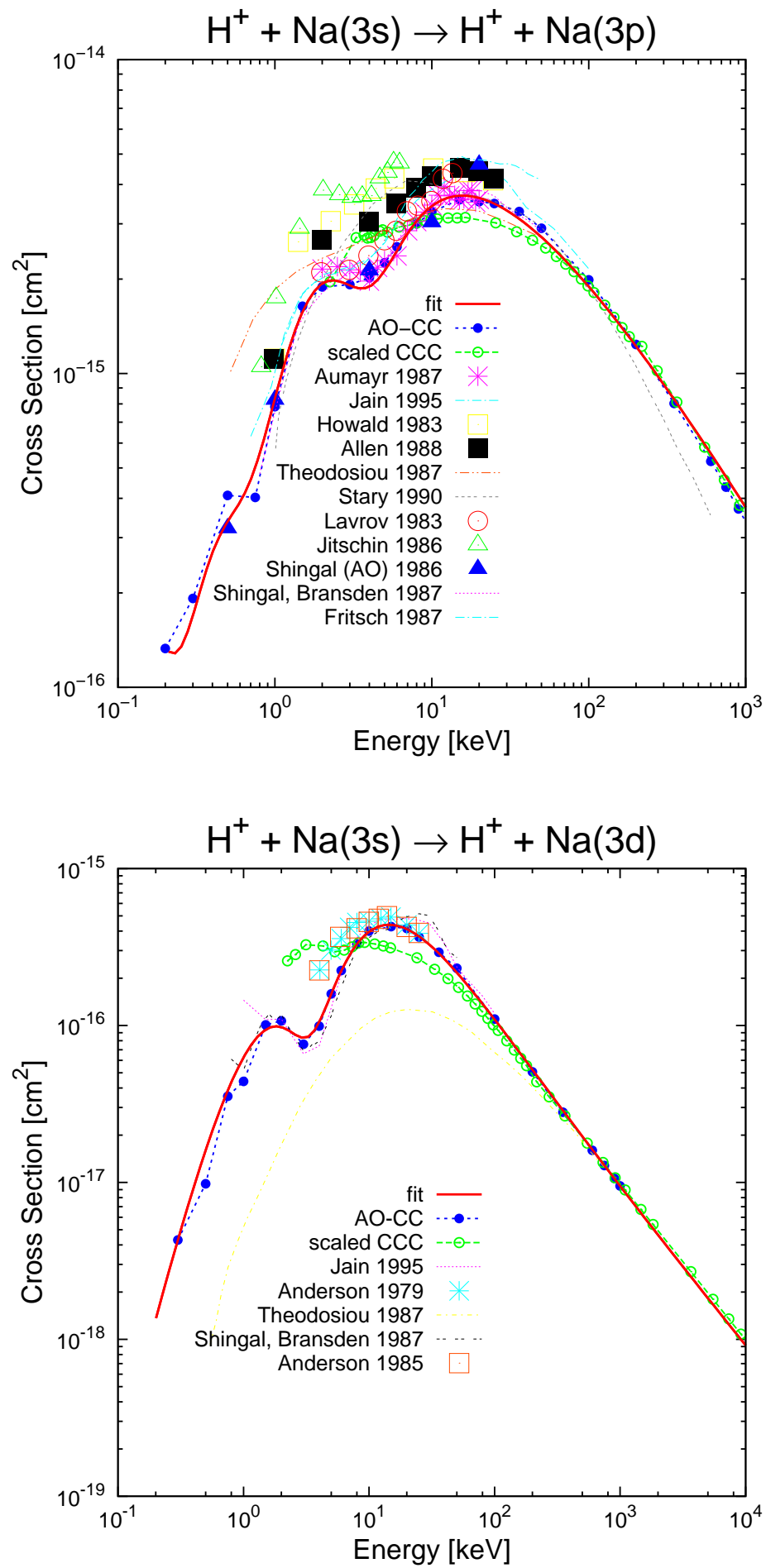

Graphs - 19 
Proton-impact target excitation (cont.)
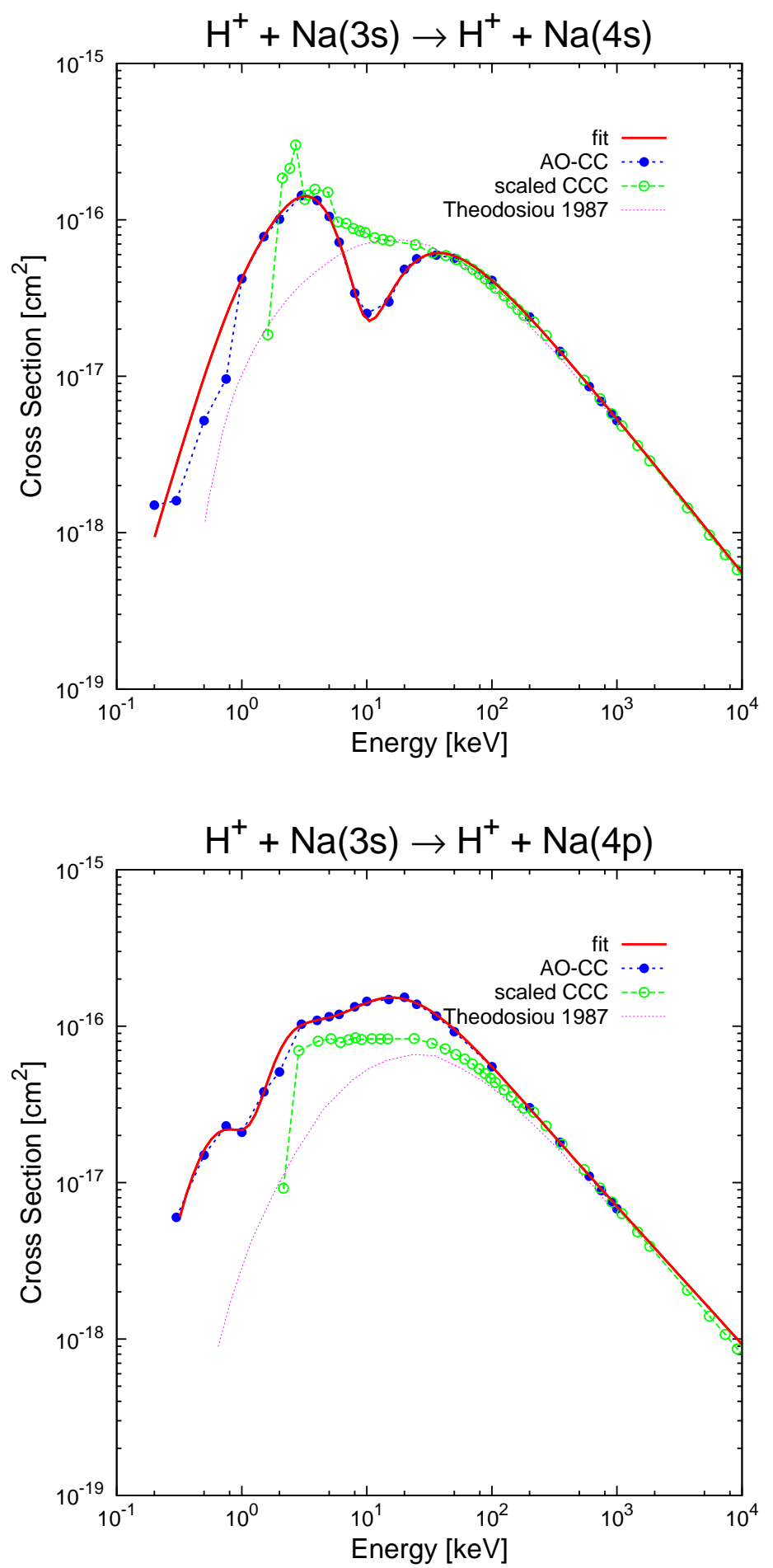

Graphs - 20 
Proton-impact target excitation (cont.)
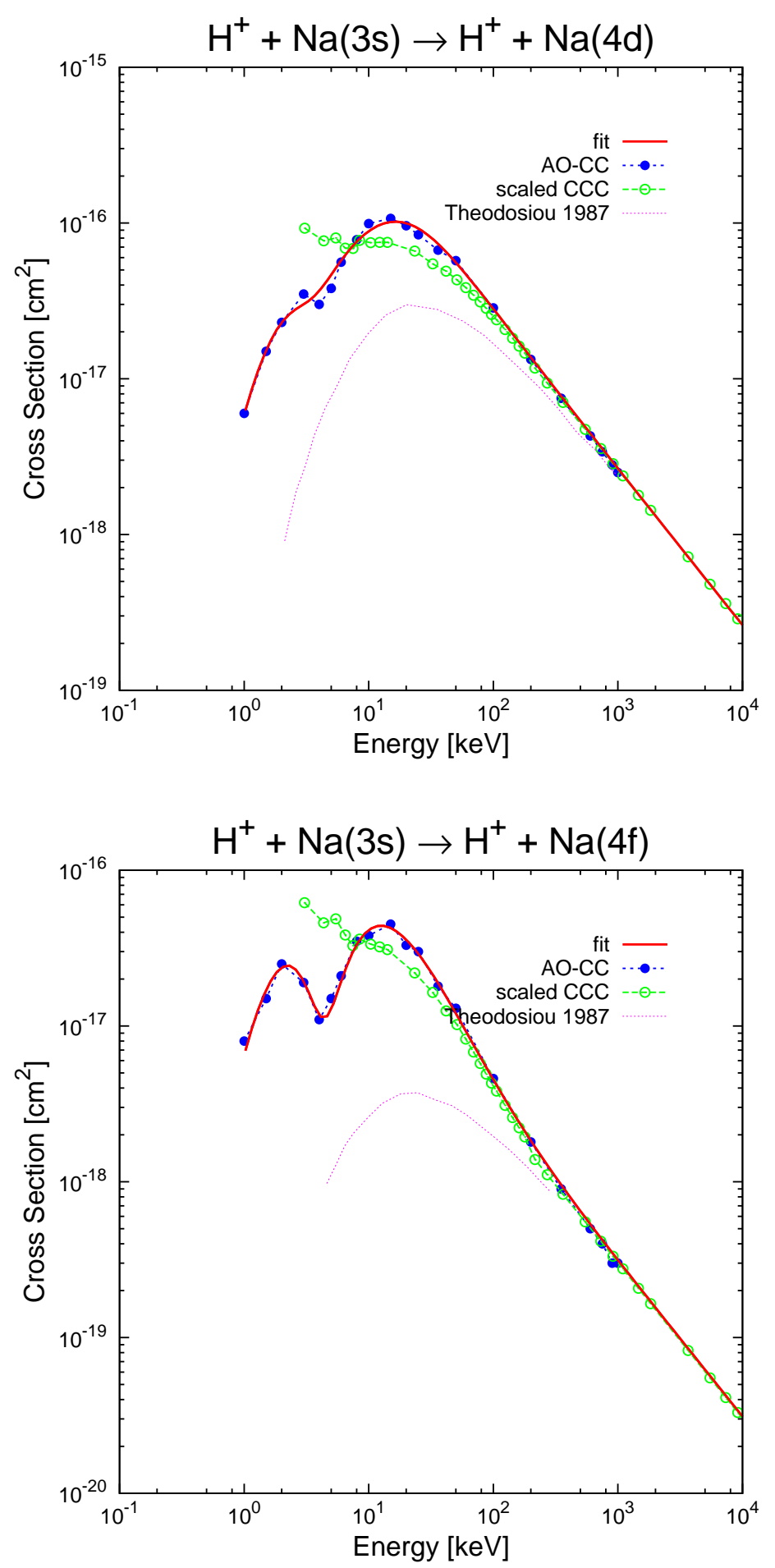

Graphs - 21 
Proton-impact target excitation (cont.)
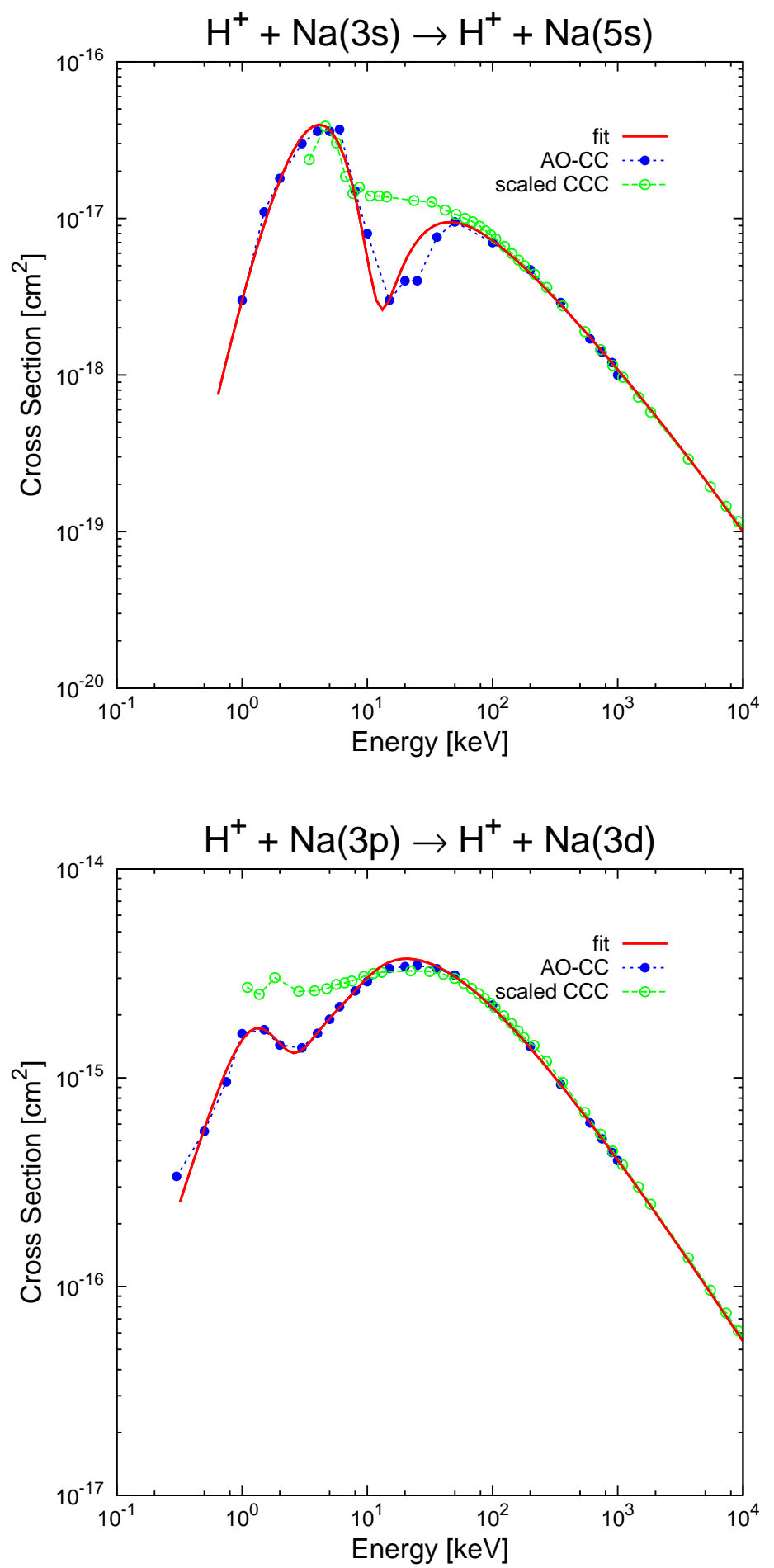

Graphs - 22 
Proton-impact target excitation (cont.)
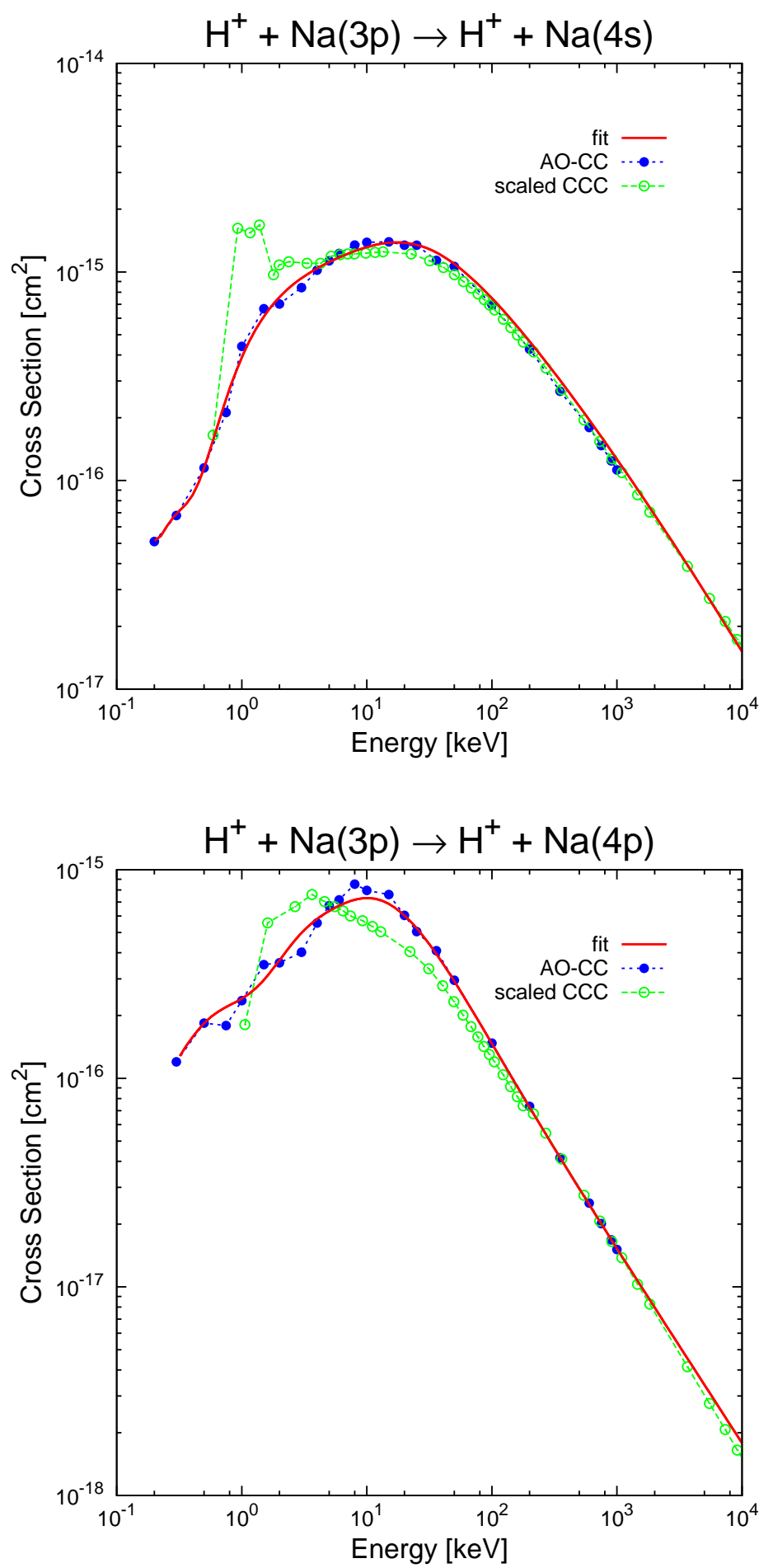

Graphs - 23 
Proton-impact target excitation (cont.)
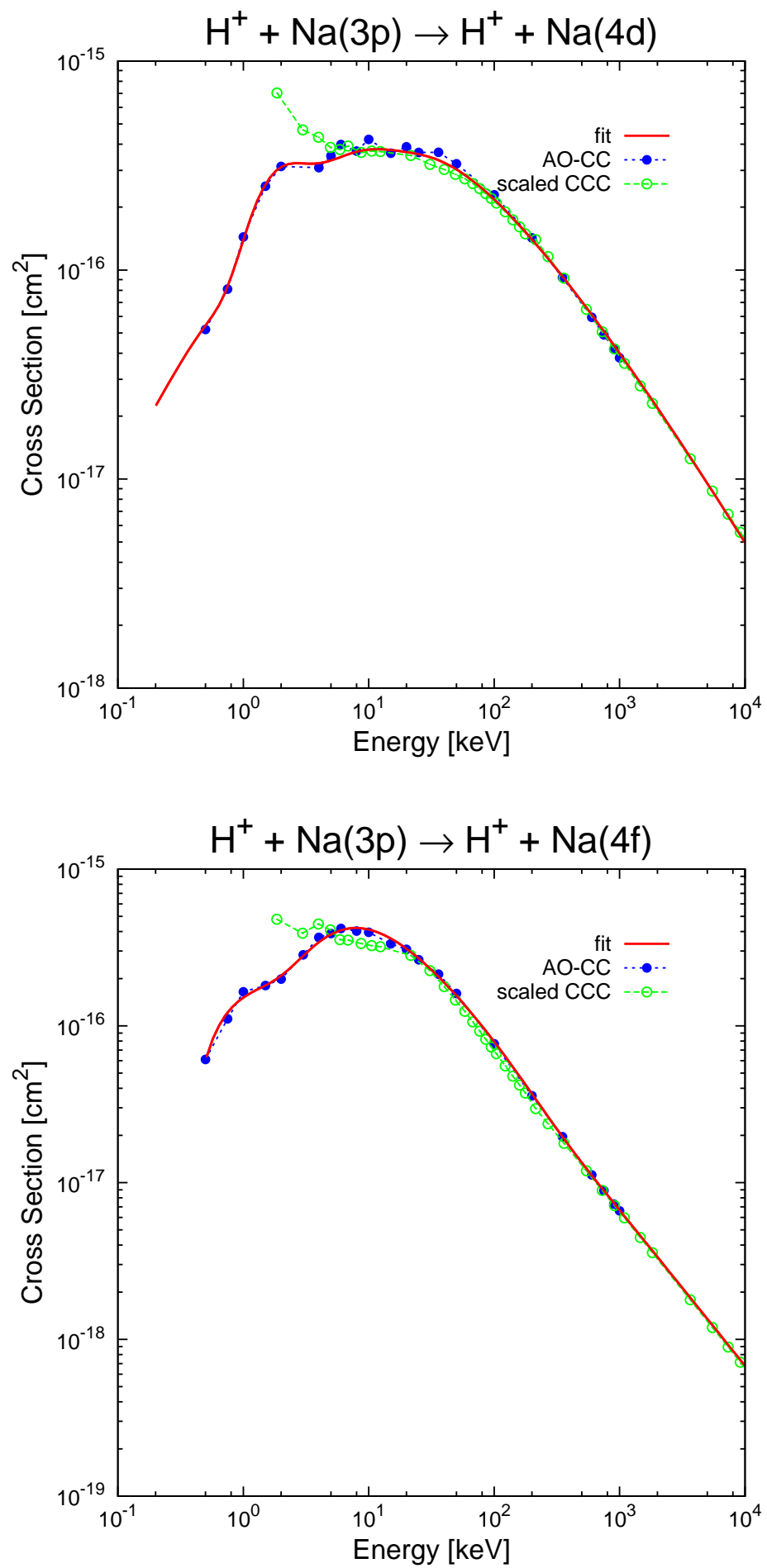

Graphs - 24 
Proton-impact target excitation (cont.)
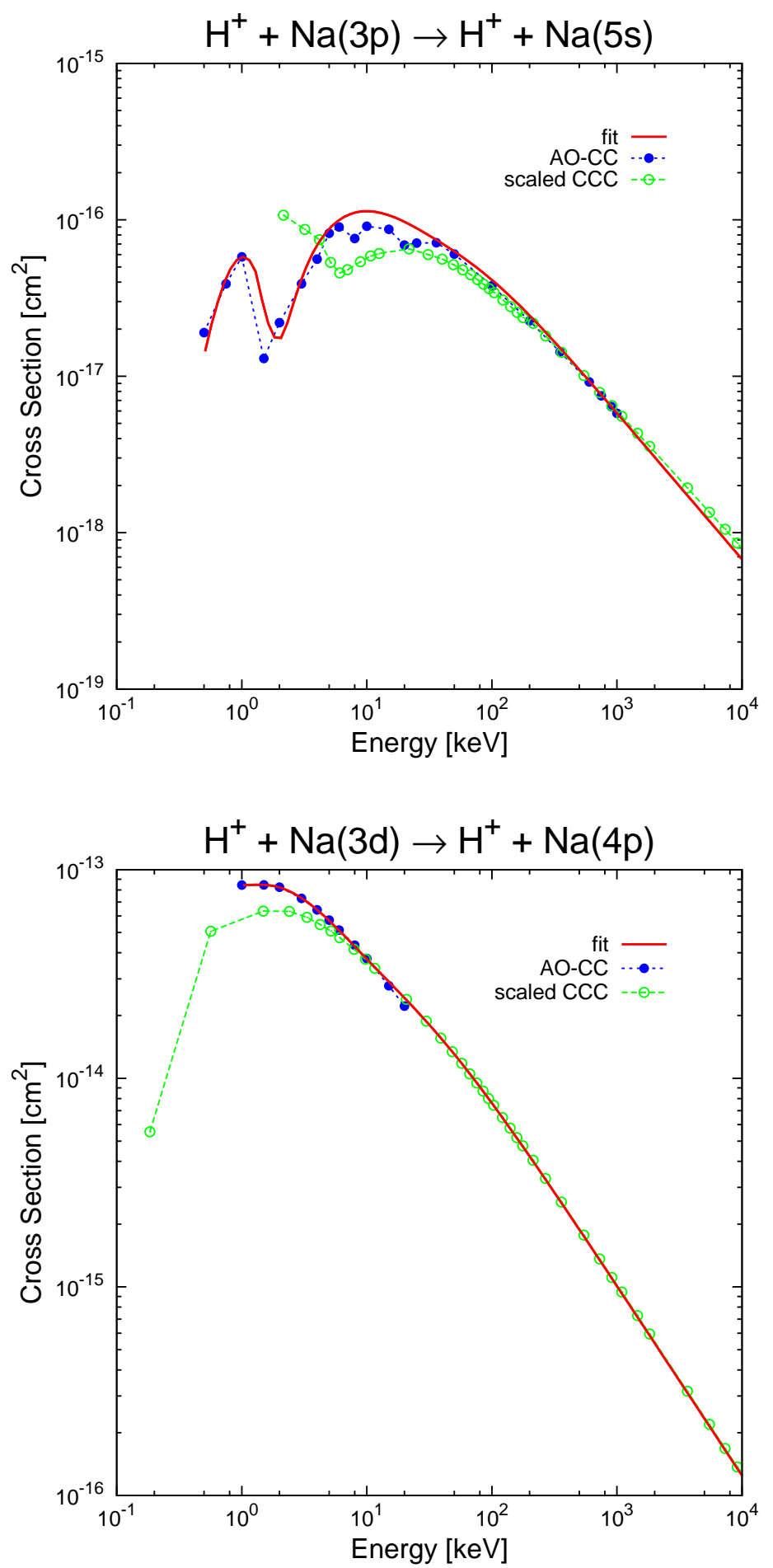

Graphs - 25 
Proton-impact target excitation (cont.)
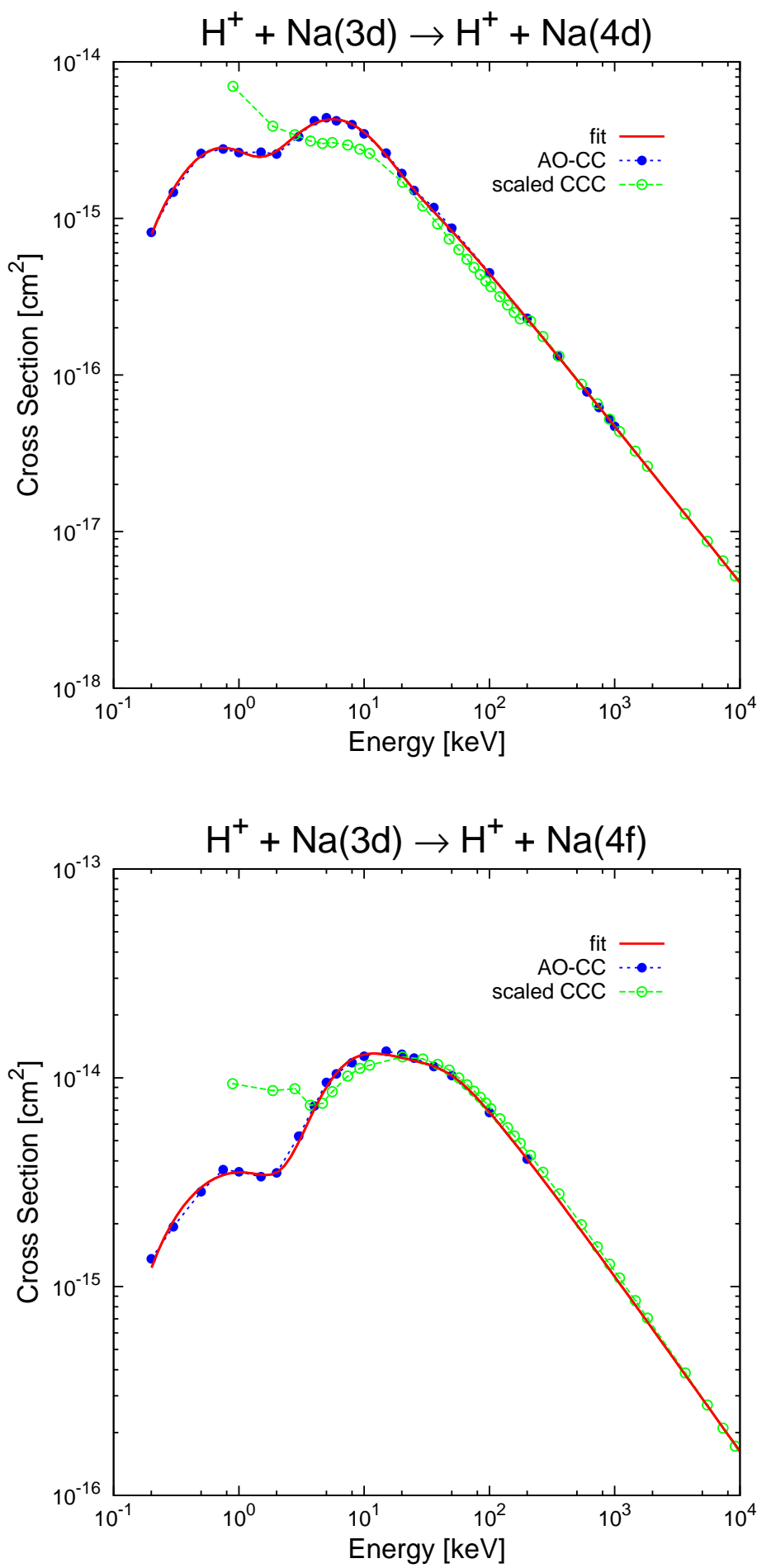

Graphs - 26 
Proton-impact target excitation (cont.)
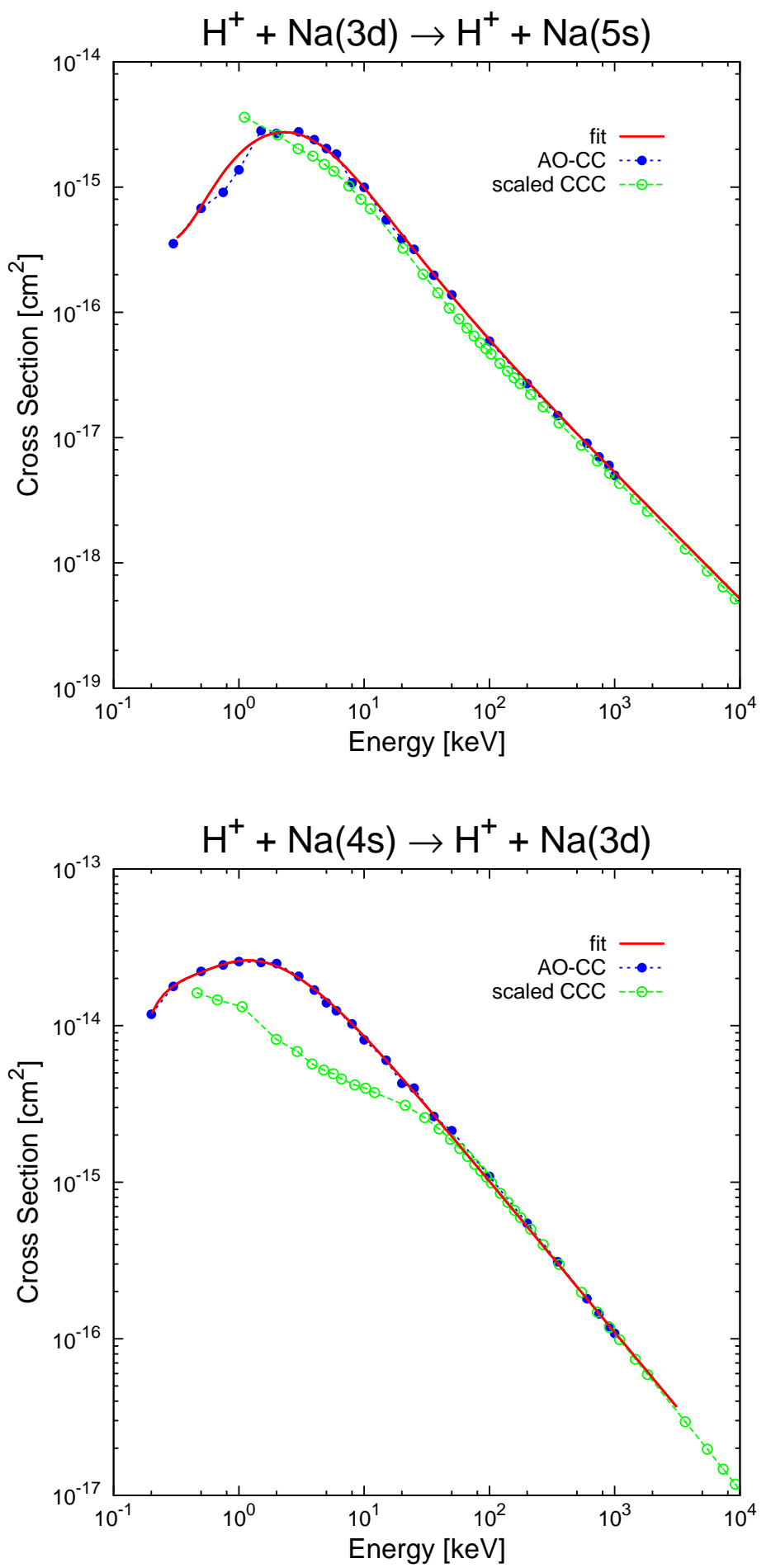

Graphs - 27 
Proton-impact target excitation (cont.)
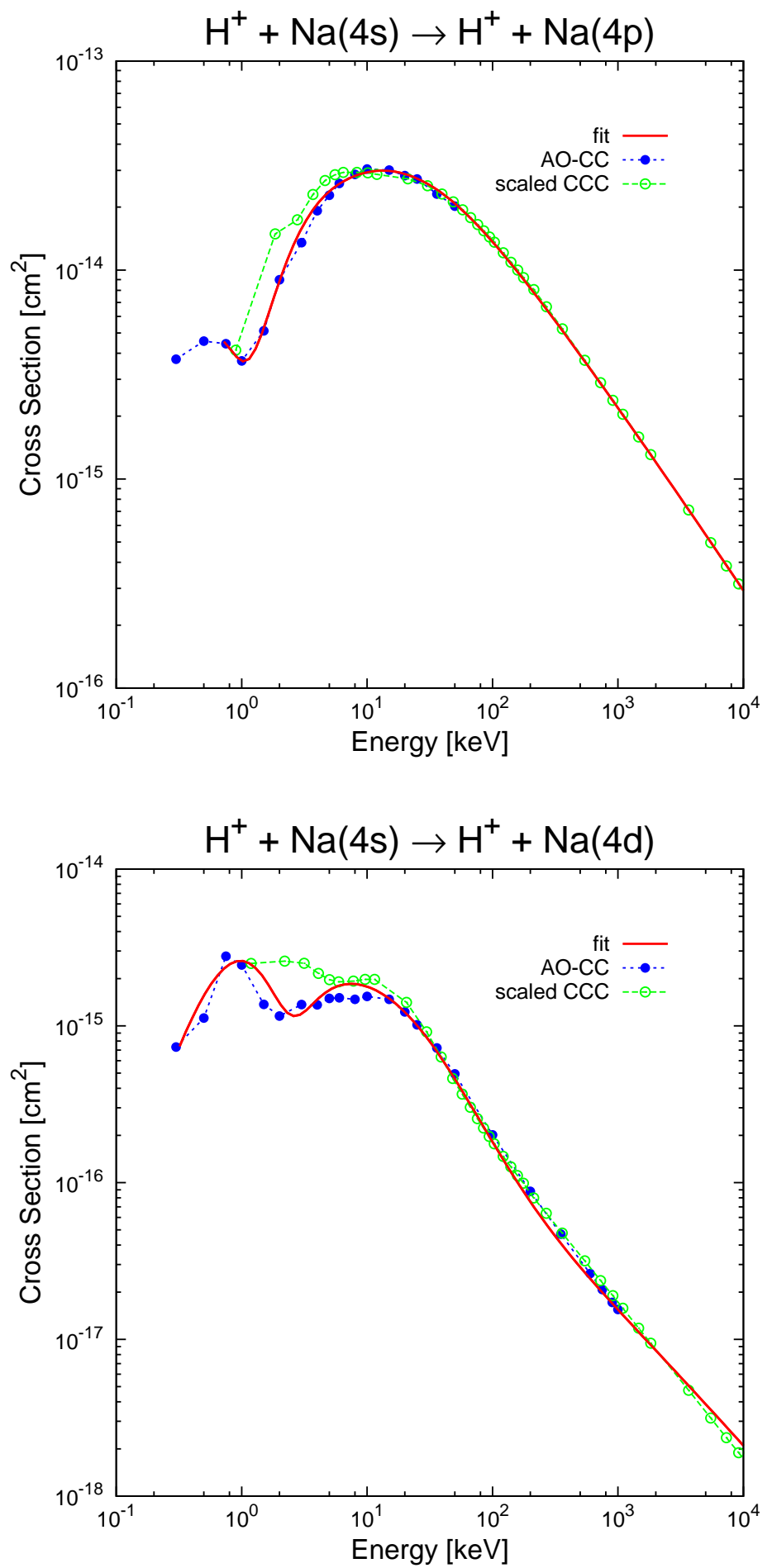

Graphs - 28 
Proton-impact target excitation (cont.)
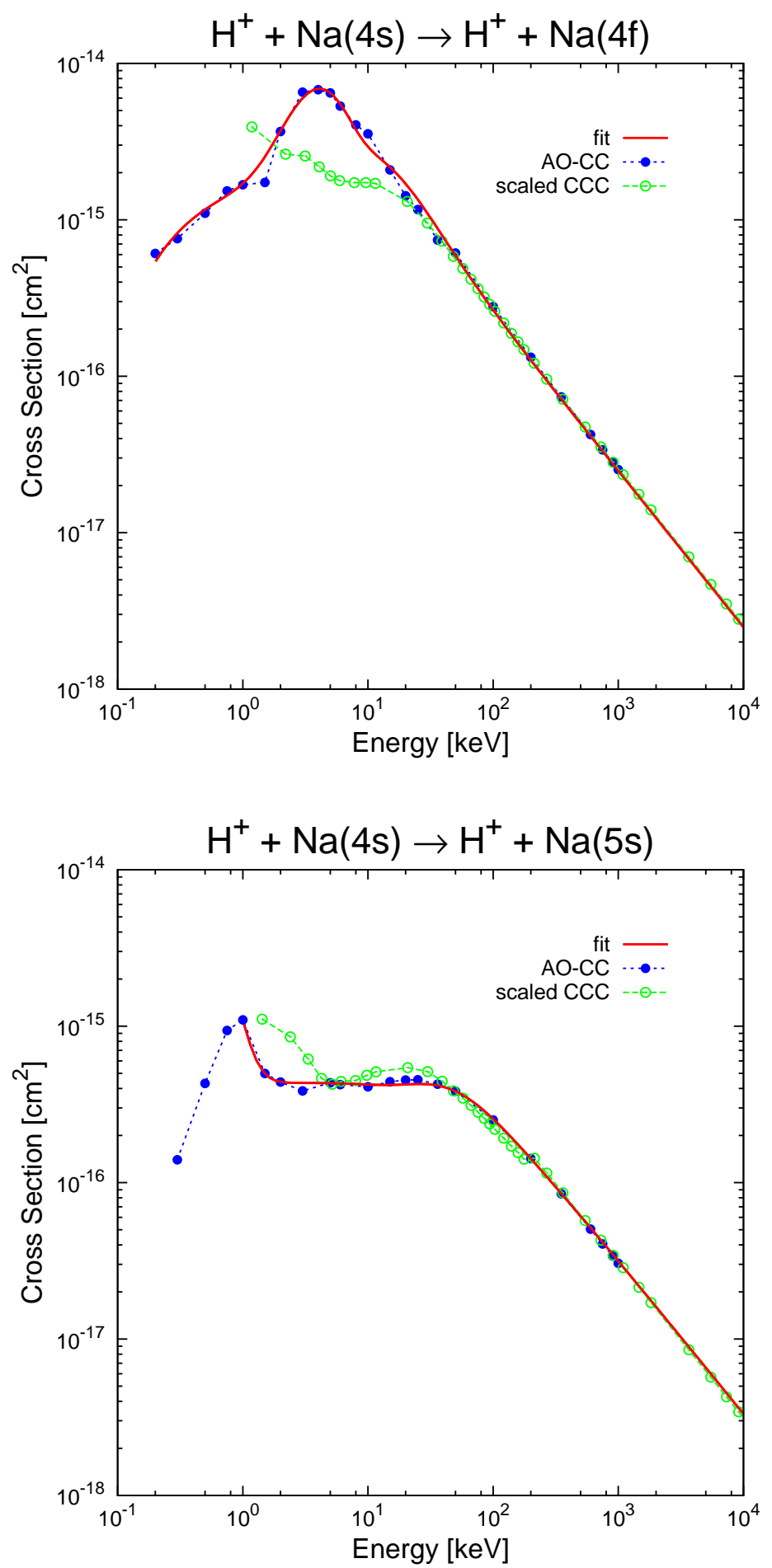

Graphs - 29 
Proton-impact target excitation (cont.)
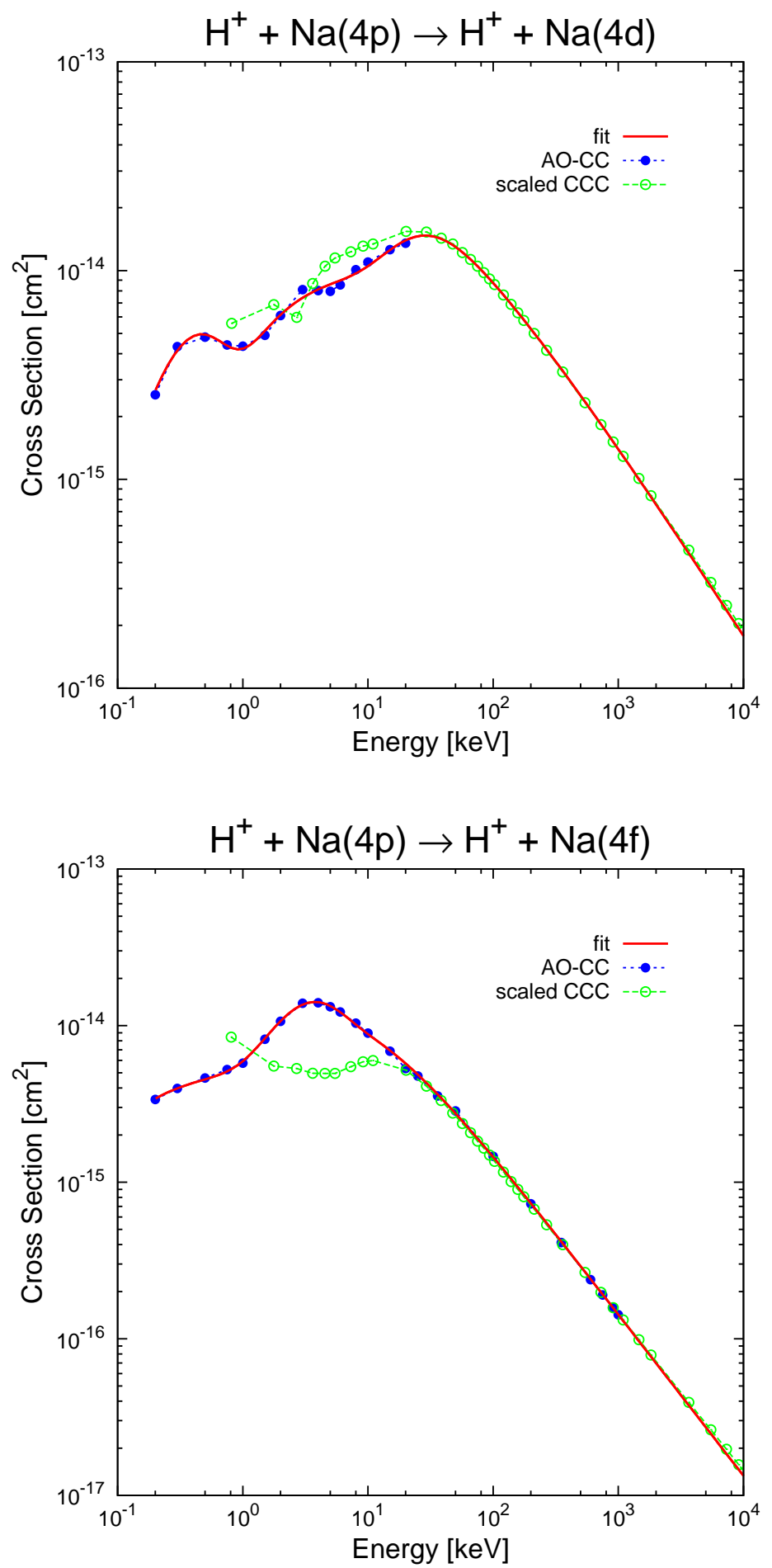

Graphs - 30 
Proton-impact target excitation (cont.)
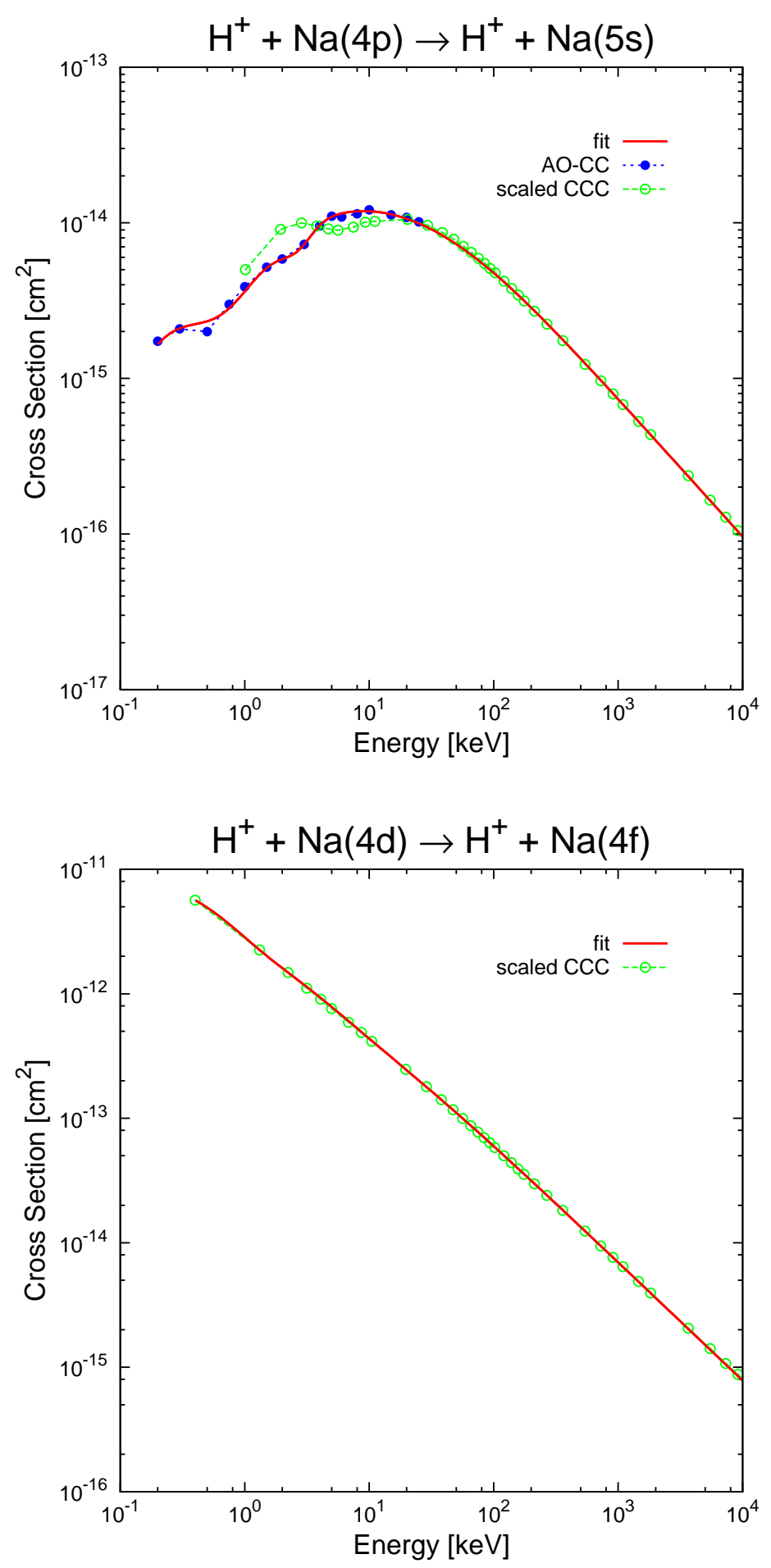

Graphs - 31 
Proton-impact target excitation (cont.)
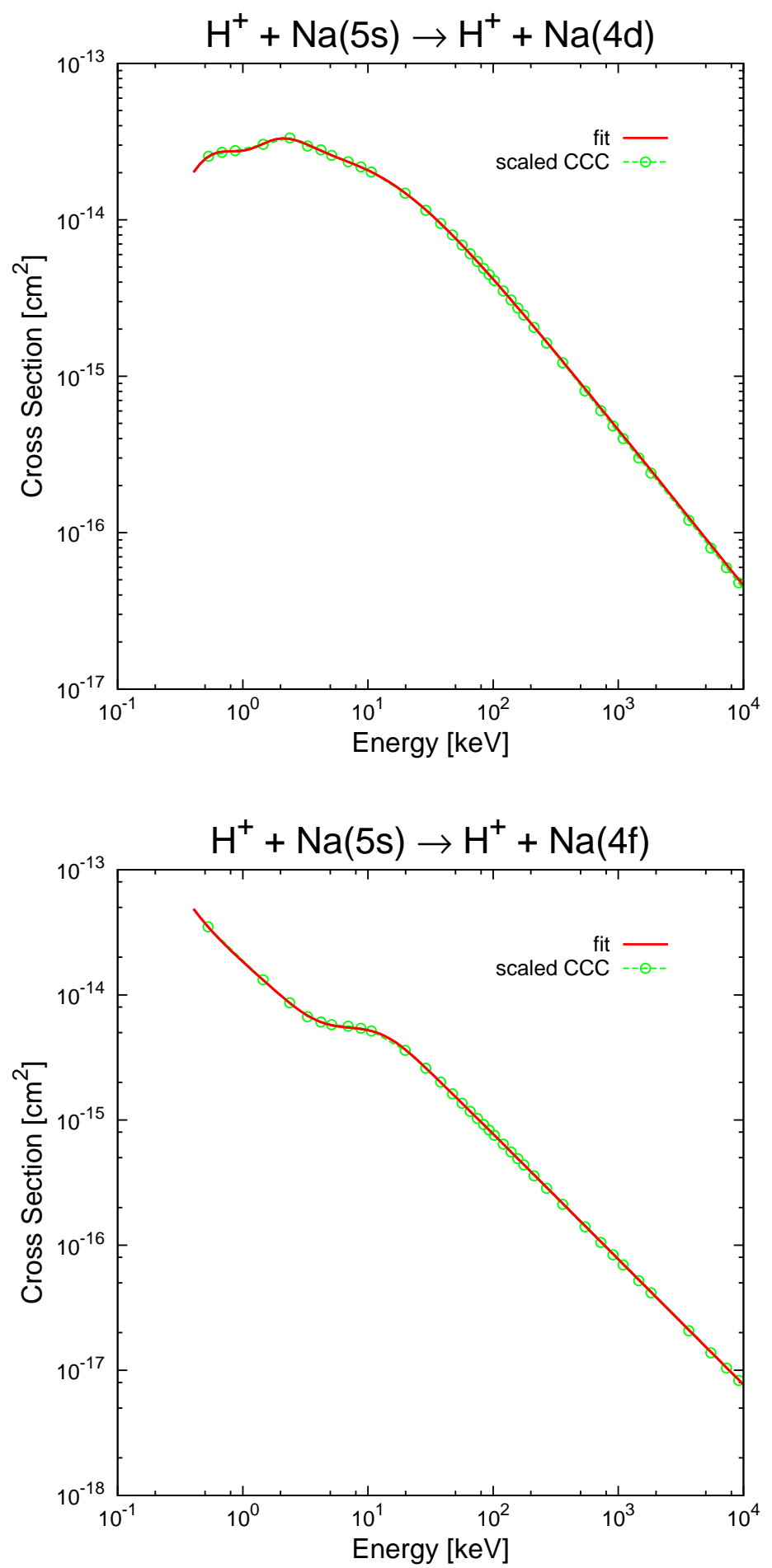

Graphs - 32 

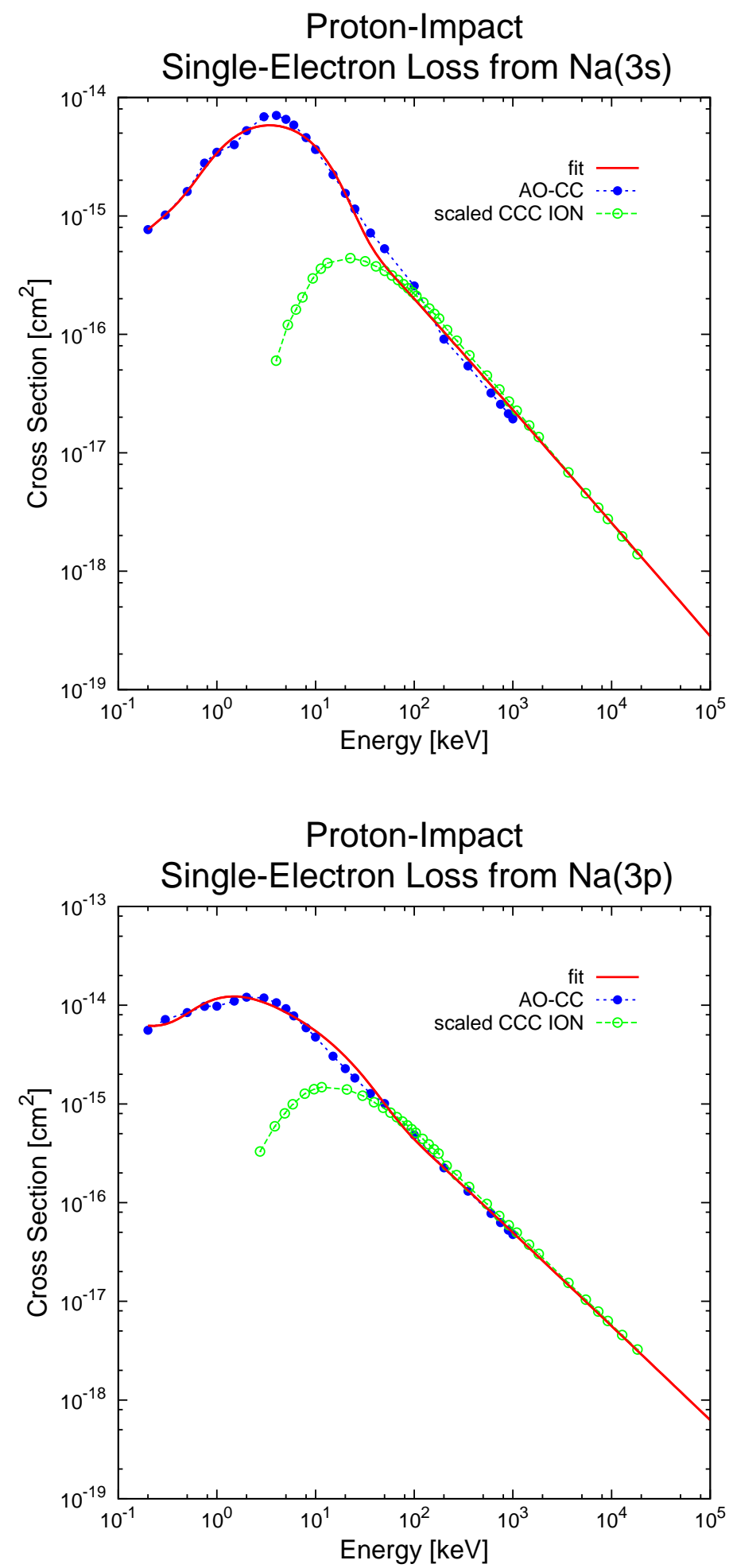

Graphs - 33 
Proton-impact target-electron-loss (cont.)
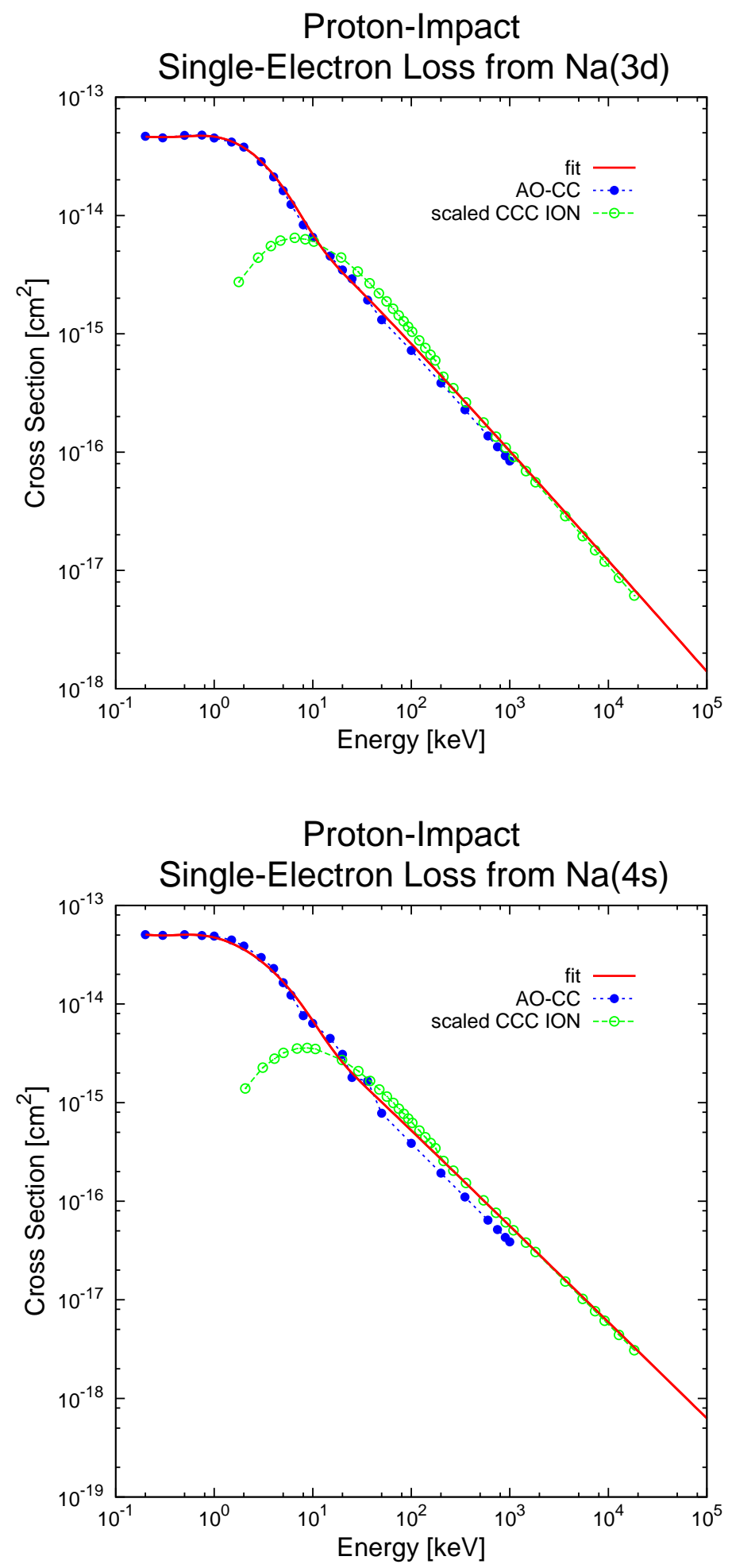

Graphs - 34 
Proton-impact target-electron-loss (cont.)
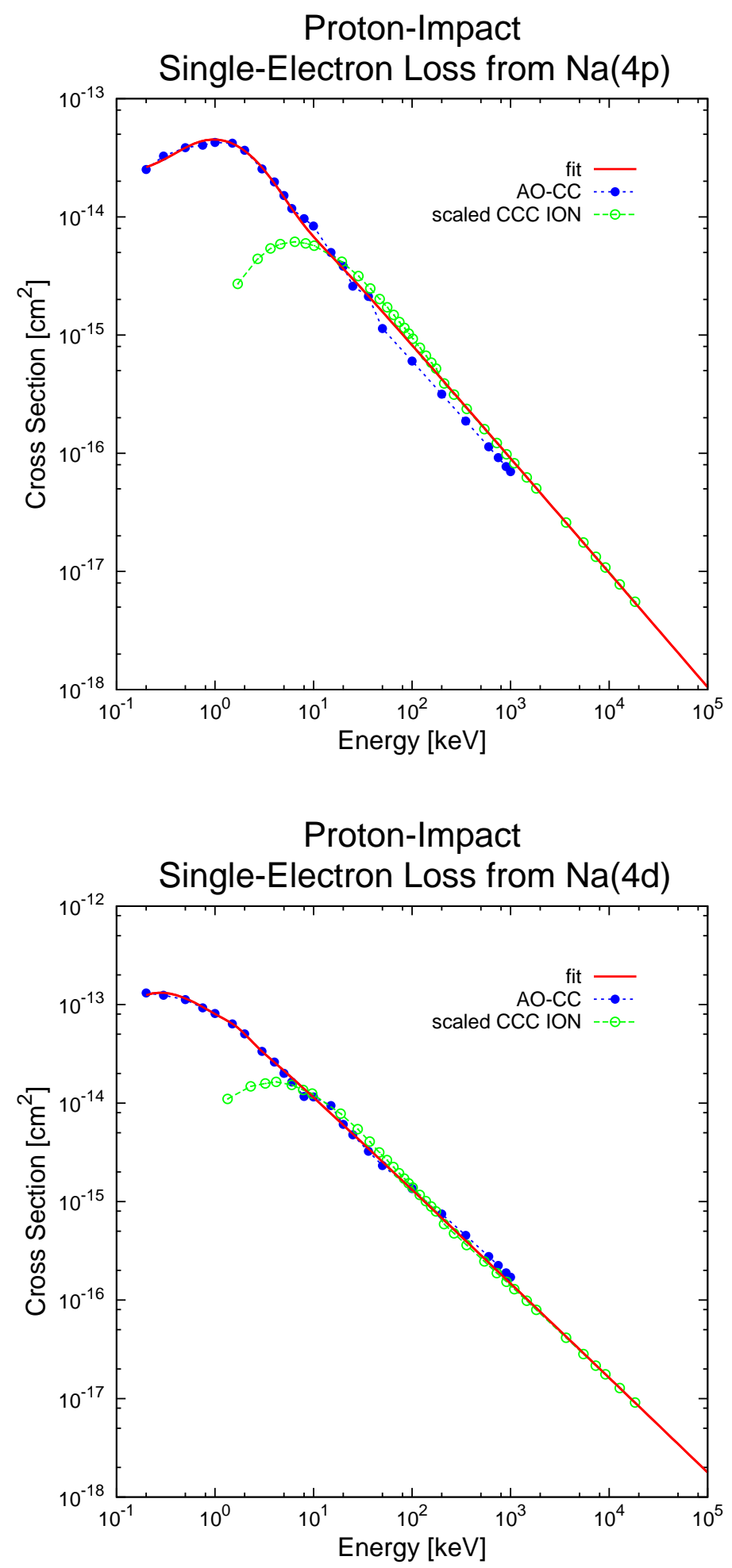

Graphs - 35 
Proton-impact target-electron-loss (cont.)
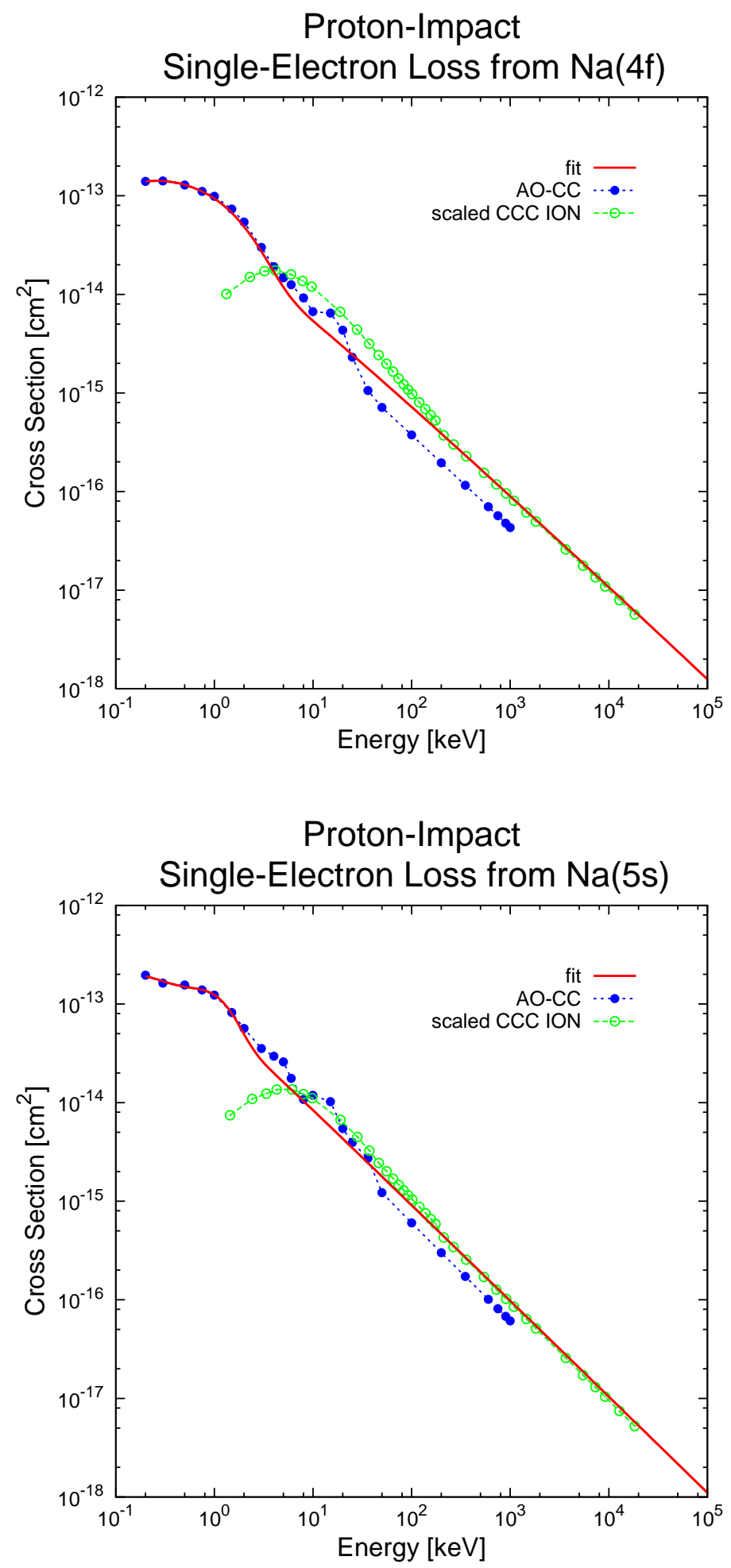

Graphs - 36 